J. DIFFERENTIAL GEOMETRY

61 (2002) 397-451

\title{
CHEEGER MANIFOLDS AND THE CLASSIFICATION OF BIQUOTIENTS
}

\author{
BURT TOTARO
}

\begin{abstract}
We classify the biquotient manifolds which are either rational homology spheres or Cheeger manifolds (connected sums of two rank-one symmetric spaces). Also: there are only finitely many 2 -connected biquotient manifolds in each dimension.
\end{abstract}

A closed manifold is called a biquotient if it is diffeomorphic to $K \backslash G / H$ for some compact Lie group $G$ with closed subgroups $K$ and $H$ such that $K$ acts freely on $G / H$. Every biquotient has a Riemannian metric of nonnegative sectional curvature. In fact, almost all known manifolds of nonnegative curvature are biquotients. The only known closed manifolds of nonnegative sectional curvature which were not defined in this way are those found by Cheeger [6] in 1973, and Grove and Ziller [16] in 2000.

In order to understand these constructions better, this paper analyzes which of the Cheeger and Grove-Ziller manifolds are actually diffeomorphic to biquotients. In the process, we develop a general approach to the classification of biquotient manifolds. A priori, it is hard to determine whether a given manifold is a biquotient, because there is no obvious upper bound on the dimension of the groups involved. We give a procedure which allows us to reduce the dimension of the groups needed to describe a given manifold as a biquotient. A surprising application is that Gromoll-Meyer's example of an exotic 7-sphere which is a biquotient [15] is the only exotic sphere of any dimension which is a biquotient. More generally, we classify all biquotients which are simply connected rational homology spheres of any dimension (Theorem 6.1). The classification of biquotients which are simply connected

Received 09/18/2002. 
rational homology spheres has been given independently by Kapovitch and Ziller [17]. More generally, they classify all simply connected biquotients whose rational cohomology ring is generated by one element.

Another application of our general classification theory for biquotients is the precise determination of which Cheeger manifolds, the connected sums of two rank-one symmetric spaces with any orientations, are diffeomorphic to biquotients (Theorem 2.1). Some of the Cheeger manifolds were known to be biquotients, such as $\mathbf{C P}^{2} \#-\mathbf{C P}^{2}$ (the nontrivial $S^{2}$-bundle over $S^{2}$ ), but we find that many of the other Cheeger manifolds are also biquotients, such as $\mathbf{C P}^{2} \# \mathbf{C P}^{2}$. On the other hand, the Cheeger manifold $\mathbf{C P}^{4} \# \mathbf{H P}^{2}$ is not diffeomorphic (or even homotopy equivalent) to a biquotient. The positive result that some Cheeger manifolds such as $\mathbf{C P} \mathbf{P}^{2} \# \mathbf{C P}^{2}$ are biquotients implies that they have nonnegatively curved Riemannian metrics with various good properties that were not clear from Cheeger's construction. First, the new metrics are real analytic. Further, the new metrics determine a natural complexanalytic structure on the whole tangent bundle, since Aguilar [2] showed that biquotients have this property; my paper [26] has a weaker result which applies to all the Cheeger manifolds. Finally, the geodesic flow for the new metrics on the Cheeger manifolds has zero topological entropy. Paternain [23] proved the latter property for Cheeger's metric on $\mathbf{C P}^{2} \# \mathbf{C P}^{2}$, but now we know it for some metric on a large class of the Cheeger manifolds.

Finally, our general classification results imply that there are only finitely many diffeomorphism classes of 2-connected biquotients in any given dimension (Theorem 4.9). This fails for biquotients that are only simply connected already in dimension 6, as I showed in [27]. Also, the finiteness of 2-connected biquotients shows the distance between biquotients and general manifolds of nonnegative sectional curvature, since Grove and Ziller have constructed nonnegatively curved metrics on all $S^{3}$-bundles over $S^{4}$, giving infinitely many homotopy types of 2-connected 7-manifolds with nonnegative sectional curvature [16].

I would like to thank Gabriel Paternain for many useful conversations. Also, thanks to Wolfgang Ziller for several references to earlier work.

\section{Notation}

We begin with the equivalence of several definitions of biquotient manifolds, pointed out by Eschenburg ([9], [11]). 
Lemma 1.1. The following properties of a closed smooth manifold $M$ are equivalent:

(1) $M=K \backslash G / H$ for some compact Lie group $G$ with closed subgroups $K$ and $H$ such that $K$ acts freely on $G / H$.

(2) $M=G / H$ for some compact Lie groups $G$ and $H$ together with a homomorphism $H \rightarrow G \times G$ such that $H$ acts freely on $G$ by left and right translation, $\left(g_{1}, g_{2}\right)(g):=g_{1} g g_{2}^{-1}$.

(3) $M=G / H$ for some compact Lie groups $G$ and $H$ together with a homomorphism $H \rightarrow(G \times G) / Z(G)$ such that $H$ acts freely on $G$.

In (3) we are using that the center $Z(G)$, imbedded diagonally in $G \times G$, acts trivially on $G$ by left and right translation.

Proof. Clearly (1) implies (2) and (2) implies (3). We prove that (3) implies (1). The point is that $(G \times G) / Z(G)$ acts transitively on $G$ with stabilizer at $1 \in G$ equal to the diagonal subgroup $G / Z(G)$. So, if $M=G / H$ as in (3), then we can also describe $M$ as in (1) by

$$
M=(G / Z(G)) \backslash((G \times G) / Z(G)) / H .
$$

q.e.d.

In the paper, we use definition (3) of biquotients; that is, "a biquotient $G / H$ " will mean that $G$ and $H$ are compact Lie groups and we are given a homomorphism $H \rightarrow(G \times G) / Z(G)$ such that $H$ acts freely on $G$. When $G$ and $H$ are simply connected, such a homomorphism lifts uniquely to $G \times G$.

We call a connected compact Lie group simple if it is nonabelian and every proper normal subgroup is finite. We define a simple factor of a connected compact Lie group $H$ to be the universal cover of a simple normal subgroup of $H$. We write $\operatorname{Sp}(2 a)$ for the simply connected simple group of type $C_{a}$, which topologists usually call $\operatorname{Sp}(a)$. The reason for the name $\operatorname{Sp}(2 a)$ is that we think of each compact classical group using its standard complex representation: $\mathrm{SU}(n)$ is a subgroup of $\mathrm{GL}(n, \mathbf{C})$, $\mathrm{SO}(n)$ is a subgroup of $\operatorname{GL}(n, \mathbf{C})$, and (with the notation here) $\operatorname{Sp}(2 a)$ is a subgroup of $\mathrm{GL}(2 a, \mathbf{C})$.

By definition, the Dynkin index of a homomorphism $H \rightarrow G$ of simply connected simple groups is the integer corresponding to the homomorphism $\pi_{3} H \rightarrow \pi_{3} G$, both groups being canonically isomorphic to Z. Dynkin computed the Dynkin index in many cases ([8], Chapter I, Section 2). Finally, we write UT $\left(S^{n}\right)$ for the unit tangent bundle of the $n$-sphere, $\operatorname{UT}\left(S^{n}\right)=\operatorname{Spin}(n+1) / \operatorname{Spin}(n-1)$. 


\section{The Cheeger manifolds that are diffeomorphic to biquotients}

By definition, a Cheeger manifold is the connected sum of any two rank-one symmetric spaces with any orientations. The rank-one symmetric spaces, besides the sphere which is not interesting for this purpose, are the real, complex, and quaternionic projective spaces, together with the Cayley plane associated to the octonions. The Cheeger manifolds are the only known examples of connected sums, with neither summand a homotopy sphere and at least one summand not a rational homology sphere, which admit metrics of nonnegative sectional curvature. In fact, the conjecture that manifolds of nonnegative sectional curvature are elliptic in the sense of Félix, Halperin, and Thomas [12] would imply that any connected sum which admits a metric of nonnegative sectional curvature must be roughly of the Cheeger type. Precisely, a connected sum $M_{1} \# M_{2}$ of simply connected manifolds which is elliptic, and such that neither $M_{1}$ nor $M_{2}$ is a $k$-homology sphere for a field $k$, must have the rings $H^{*}\left(M_{1}, k\right)$ and $H^{*}\left(M_{2}, k\right)$ both generated by a single element, as follows from Lambrechts [18], Theorem 3. Combining this with Adams and Atiyah's results on the Hopf invariant problem [1] shows that if a connected sum of simply connected manifolds is elliptic, with neither summand a homotopy sphere and at least one summand not a rational homology sphere, then both summands have the integral cohomology ring of $\mathbf{C P}^{n}, \mathbf{H} \mathbf{P}^{n}$, or $\mathbf{C a P} \mathbf{P}^{2}$.

Theorem 2.1. The following Cheeger manifolds are diffeomor phic to biquotients. First, $\mathbf{C P}^{n} \#-\mathbf{C P}^{n}, \mathbf{H P}^{n} \#-\mathbf{H P}^{n}$, and $\mathbf{C a P}^{2} \#-\mathbf{C a P}^{2}$. Next, $\mathbf{R} \mathbf{P}^{n} \# \mathbf{R} \mathbf{P}^{n}, \mathbf{R P}^{2 n} \# \mathbf{C P}^{n}, \mathbf{R} \mathbf{P}^{4 n} \# \mathbf{H P}^{n}$, and $\mathbf{R} \mathbf{P}^{16} \# \mathbf{C a} \mathbf{P}^{2}$. Here $\mathbf{R P}^{n}$ is non-orientable for $n$ odd and has an orientation-reversing diffeomorphism for $n$ even, so the orientations of the summands do not matter in these cases. Next, $\mathbf{C P}^{2 n} \#-\mathbf{H P}^{n}, \mathbf{H P}^{4} \#-$ $\mathbf{C a P}{ }^{2}$, and $\mathbf{C P}^{8} \#-\mathbf{C a P}^{2}$. Finally, $\mathbf{C P}^{n} \# \mathbf{C P}^{n}, \mathbf{H P}^{n} \# \mathbf{H} \mathbf{P}^{n}$, and $\mathbf{C P}^{4 e+2} \# \mathbf{H P}^{2 e+1}$.

The remaining Cheeger manifolds are not even homotopy equivalent to biquotients. These are $\mathbf{C a P}^{2} \# \mathbf{C a P} \mathbf{P}^{2}, \mathbf{C P}^{8} \# \mathbf{C a} \mathbf{P}^{2}, \mathbf{H} \mathbf{P}^{4} \# \mathbf{C a P}^{2}$, and $\mathbf{C P}^{4 e} \# \mathbf{H} \mathbf{P}^{2 e}$.

Proof. In this section, we prove only the first, positive, statement. We will prove the negative statement in Sections 7 and 8, after setting up a general classification theory of biquotients.

We begin with the cases which are straightforward generalizations 
of Cheeger's observation that $\mathbf{C P}^{2} \#-\mathbf{C P}^{2}$ is a biquotient [6]. Let $A_{k}$ denote the standard algebra $(\mathbf{R}, \mathbf{C}, \mathbf{H}$, or the octonions $\mathbf{C a})$ of dimension $k$ over $\mathbf{R}$, where $k=1,2,4$, or 8 . Then, for $k=1,2$, or 4 , $A_{k} \mathbf{P}^{n} \#-A_{k} \mathbf{P}^{n}$ is an $S^{k}$-bundle over $A_{k} \mathbf{P}^{n-1}$, namely the biquotient manifold $\left(S^{n k-1} \times S^{k}\right) / S^{k-1}$, where $S^{k-1}$ is a group for $k=1,2$, or 4 , acting freely on $S^{n k-1}$ and by rotations on $S^{k}$. For $k=8, S^{7}$ is not a group, but we can still describe $\mathbf{C a P}^{2} \#-\mathbf{C a P}^{2}$ as a biquotient,

$$
\left(\operatorname{Spin}(9) \times S^{8}\right) / \operatorname{Spin}(8),
$$

where $\operatorname{Spin}(8) \rightarrow \operatorname{Spin}(9)$ is the standard inclusion and $\operatorname{Spin}(8)$ acts on $S^{8} \subset \mathbf{R}^{9}$ by the direct sum of an 8 -dimensional real spin representation and the trivial representation. Since $\operatorname{Spin}(9) / \operatorname{Spin}(8)=S^{8}$, this description exhibits $\mathbf{C a P}^{2} \#-\mathbf{C a P}^{2}$ as an $S^{8}$-bundle over $S^{8}$.

Next, we check that the connected sum of $\mathbf{R P}^{n}$ with any rank-one symmetric space is diffeomorphic to a biquotient. As mentioned in the theorem, orientations do not matter in this case, because $\mathbf{R P}^{n}$ is nonorientable for $n$ odd and has an orientation-reversing diffeomorphism for $n$ even. For any closed $n$-manifold $M$, the connected sum $\mathbf{R P}^{n} \# M$ is doubly covered by $M \#-M$. This suggests a way to view $\mathbf{R} \mathbf{P}^{n k} \# A_{k} \mathbf{P}^{n}$ as a biquotient: we replace $S^{k}$ in the above description of $A_{k} \mathbf{P}^{n} \#-$ $A_{k} \mathbf{P}^{n}$ by $\mathbf{R} \mathbf{P}^{k}$. That is:

$$
\begin{aligned}
\mathbf{R} \mathbf{P}^{n} \# \mathbf{R} \mathbf{P}^{n} & =\left(S^{n-1} \times S^{1}\right) / \mathbf{Z} / 2 \\
\mathbf{R} \mathbf{P}^{2 n} \# \mathbf{C P}^{n} & =\left(S^{2 n-1} \times \mathbf{R} \mathbf{P}^{2}\right) / S^{1} \\
\mathbf{R} \mathbf{P}^{4 n} \# \mathbf{H} \mathbf{P}^{n} & =\left(S^{4 n-1} \times \mathbf{R} \mathbf{P}^{4}\right) / S^{3} \\
\mathbf{R} \mathbf{P}^{16} \# \mathbf{C a P} \mathbf{P}^{2} & =\left(\operatorname{Spin}(9) \times \mathbf{R} \mathbf{P}^{8}\right) / \operatorname{Spin}(8)
\end{aligned}
$$

The manifolds $\mathbf{C} \mathbf{P}^{2 n}, \mathbf{H P}^{2 n}$, and $\mathbf{C a P} \mathbf{P}^{2}$ have natural orientations, corresponding to the highest power of any generator of $H^{2}, H^{4}$, or $H^{8}$, respectively. In fact, $\mathbf{H P}^{n}$ has a natural orientation for all $n \geq 2$, but this takes more care to define. We use that the mod 3 Steenrod operation $P^{1}$ on $H^{*}\left(\mathrm{BSU}(2), \mathbf{F}_{3}\right)$ acts by $P^{1} c_{2}=c_{2}^{2}$, as one checks by restricting to the torus $S^{1} \subset \mathrm{SU}(2)$. Since $H^{4}\left(\mathbf{H P}^{n}, \mathbf{Z}\right)$ has a generator which is $c_{2}$ of an $\mathrm{SU}(2)$-bundle over $\mathbf{H} \mathbf{P}^{n}$, this generator $c_{2}$ satisfies $P^{1} c_{2}=c_{2}^{2}$ in $H^{8}\left(\mathbf{H P}^{n}, \mathbf{F}_{3}\right)$. It follows that (for $n \geq 2$ ) there is a unique generator $z$ of $H^{4}\left(\mathbf{H P}^{n}, \mathbf{Z}\right)$ such that $P^{1} z=-z^{2}$ in $H^{8}\left(\mathbf{H P}^{n}, \mathbf{F}_{3}\right)$, namely $z=-c_{2}$. We define the natural orientation on $\mathbf{H P}^{n}$ to be the one corresponding to the highest power $z^{n}$ of this generator $z$.

Using this orientation, we can define one more class of Cheeger manifolds as bundles. The manifold $\mathbf{C P} \mathbf{P}^{2 n} \#-\mathbf{H} \mathbf{P}^{n}$ is the $\mathbf{C} \mathbf{P}^{2}$-bundle over 
$\mathbf{H P}^{n-1}$ associated to the rank-3 complex vector bundle $E \oplus \mathbf{C}$, where $E$ is the tautological rank-2 complex vector bundle over $\mathbf{H P}^{n-1}$. Thus $\mathbf{C P}^{2 n} \#-\mathbf{H} \mathbf{P}^{n}$ is a biquotient of the form $\left(\mathbf{C P}^{2} \times S^{4 n-1}\right) / \mathrm{SU}(2)$. We might try to imitate this construction by viewing $\mathbf{H P}^{4} \#-\mathbf{C a P}^{2}$ as an $\mathbf{H} \mathbf{P}^{2}$-bundle over $S^{8}$ and $\mathbf{C P}^{8} \#-\mathbf{C a P}^{2}$ as a $\mathbf{C P}^{4}$-bundle over $S^{8}$, but that turns out to be impossible. For these constructions, we would need a complex vector bundle $E$ over $S^{8}$ with $c_{4} E$ equal to plus or minus the class of a point in $H^{8}\left(S^{8}, \mathbf{Z}\right)$, whereas in fact every complex vector bundle on $S^{8}$ has $c_{4} E$ a multiple of $(4-1) !=6$, by Bott periodicity.

Instead, we construct $\mathbf{H P}^{4} \#-\mathbf{C a P}^{2}$ as the quotient of an $S^{11}$ bundle over $S^{8}$ by a free $\mathrm{SU}(2)$-action. Let $S^{-}: \mathrm{Spin}(8) \rightarrow \mathrm{SO}(8)$ denote one of the two spin representations. We also write $S^{-}$for the associated $\operatorname{Spin}(9)$-equivariant real vector bundle of rank 8 over $\operatorname{Spin}(9) / \operatorname{Spin}(8)=$ $S^{8}$. Let $N$ be the $S^{11}$-bundle over $S^{8}$ defined as the unit sphere bundle $S\left(S^{-} \oplus \mathbf{R}^{4}\right)$. Define a homomorphism $\mathrm{SU}(2) \rightarrow \operatorname{Spin}(9)$ by $\mathrm{SU}(2) \cong$ $\operatorname{Spin}(3) \subset \operatorname{Spin}(9)$. Using this homomorphism, $\mathrm{SU}(2)$ acts on $S^{8}$ and acts compatibly on the vector bundle $S^{-}$over $S^{8}$. Let $\mathrm{SU}(2)$ act on $N$ by the given action on $S^{8}$ and on the vector bundle $S^{-}$, and by the standard faithful representation $V_{\mathbf{R}}$ of $\mathrm{SU}(2)$ on $\mathbf{R}^{4}$.

This action of $\mathrm{SU}(2)$ on $N$ is free. To check this, it suffices to check that $\mathrm{SU}(2)$ acts freely on the $S^{7}$-bundle $S\left(S^{-}\right)$over $S^{8}$ and on the $S^{3}$ bundle $S\left(\mathbf{R}^{4}\right)=S^{3} \times S^{8}$ over $S^{8}$. The second statement is clear by the choice of $\mathrm{SU}(2)$-action on $\mathbf{R}^{4}$. To prove the first statement, first note that $\operatorname{Spin}(9)$ acts on $S\left(S^{-}\right) \cong S^{15}$ by the spin representation of $\operatorname{Spin}(9)$. Then use that the restriction of any spin representation of $\operatorname{Spin}(n)$ to $\operatorname{Spin}(n-1)$ is a sum of spin representations of $\operatorname{Spin}(n-1)$. Thus the action of $\mathrm{SU}(2) \cong \operatorname{Spin}(3)$ on $S\left(S^{-}\right)=S^{15}$ is by a sum of copies of the 4-dimensional real spin representation $V_{\mathbf{R}}$. It follows that $\mathrm{SU}(2)$ acts freely on $S^{15}$.

Let $M$ be the quotient manifold $N / \mathrm{SU}(2)$, of dimension 16 . Clearly $M$ is a biquotient of the form $\left(\operatorname{Spin}(9) \times S^{11}\right) /(\operatorname{Spin}(8) \times \mathrm{SU}(2))$. By construction, $M$ is the union of a disc bundle over $S\left(S^{-}\right) / \mathrm{SU}(2)=$ $S^{15} / \mathrm{SU}(2)=\mathbf{H P}^{3}$ and a disc bundle over $S\left(\mathbf{R}^{4}\right) / \mathrm{SU}(2)=\left(S^{8} \times\right.$ $\left.S^{3}\right) / \mathrm{SU}(2)=S^{8}$ along their common boundary, $S^{15}$. So $M$ is diffeomorphic to the connected sum of $\mathbf{H P}^{4}$ and $\mathbf{C a P}^{2}$, with some orientations. To pin down the orientations, it is convenient to compute the cohomology ring of $M$. Since $N$ is an SU(2)-equivariant $S^{11}$-bundle over $S^{8}$, the quotient $M$ is the total space of a fibration $S^{11} \rightarrow M \rightarrow S^{8} / / \mathrm{SU}(2)$ up to homotopy, where $S^{8} / / \mathrm{SU}(2)=\left(S^{8} \times \mathrm{ESU}(2)\right) / \mathrm{SU}(2)$ denotes the homotopy quotient via the given homomorphism $\mathrm{SU}(2) \rightarrow \operatorname{Spin}(9)$. 
We compute that

$$
H^{*}\left(S^{8} / / \mathrm{SU}(2), \mathbf{Z}\right)=\mathbf{Z}\left[y, \chi\left(S^{-}\right)\right] /\left(\left(\chi\left(S^{-}\right)+y^{2}\right)^{2}=0\right),
$$

where $y$ in $H^{4}$ is $c_{2}$ of the standard representation $V$ of $\mathrm{SU}(2)$ and $\chi\left(S^{-}\right)$ in $H^{8}$ is the Euler class of the $\mathrm{SU}(2)$-equivariant vector bundle $S^{-}$over $S^{8}$, with some orientation. To check this, note that the spectral sequence for the fibration $S^{8} \rightarrow S^{8} / / \mathrm{SU}(2) \rightarrow \mathrm{BSU}(2)$ degenerates, since all the cohomology is in even degrees. This implies that the cohomology of $S^{8} / / \mathrm{SU}(2)$ has the above form, for some relation of the form $\chi\left(S^{-}\right)^{2}+$ $b \chi\left(S^{-}\right) y^{2}+c y^{4}=0$ with $b, c \in \mathbf{Z}$. Furthermore, we have $T S^{8} \oplus \mathbf{R} \cong \mathbf{R}^{9}$ as $\mathrm{SU}(2)$-equivariant vector bundles on $S^{8}$. It follows that

$$
\chi\left(T S^{8}\right)^{2}=c_{8}\left(T S^{8} \otimes_{\mathbf{R}} \mathbf{C}\right)=0
$$

in $H^{16}\left(S^{8} / / \mathrm{SU}(2), \mathbf{Z}\right)$. Also, $\chi\left(T S^{8}\right)$ has degree 2 on $S^{8}$ while $\chi\left(S^{-}\right)$ has degree \pm 1 on $S^{8}$, so we must have $\chi\left(T S^{8}\right)= \pm 2 \chi\left(S^{-}\right)+d y^{2}$ in $H^{8}\left(S^{8} / / \mathrm{SU}(2), \mathbf{Z}\right)$ for some integer $d$. So $\left( \pm \chi\left(S^{-}\right)+(d / 2) y^{2}\right)^{2}=0$ in $H^{16}\left(S^{8} / / \mathrm{SU}(2), \mathbf{Q}\right)$. By what we know about the form of the relation, $d / 2$ must be an integer $a$, and we have

$$
\left( \pm \chi\left(S^{-}\right)+a y^{2}\right)^{2}=0
$$

in $H^{16}\left(S^{8} / / \mathrm{SU}(2), \mathbf{Z}\right)$. Finally, the action of $\mathrm{SU}(2)=\operatorname{Spin}(3) \subset \operatorname{Spin}(9)$ on $S^{8}$ preserves a 2 -sphere, and so we have an inclusion $\mathrm{B} S^{1} \simeq S^{2} / / \mathrm{SU}(2)$ $\rightarrow S^{8} / / \mathrm{SU}(2)$. Let $x$ be the standard generator of the polynomial ring $H^{*}\left(\mathrm{~B} S^{1}, \mathbf{Z}\right)$ in degree 2 . We compute that the restriction map takes $y=c_{2} V$ to $-x^{2}$ and $\chi\left(S^{-}\right)$to $\pm x^{4}$, using that the restriction of the spin representation $S^{-}$to $S^{1}=\operatorname{Spin}(2) \subset \operatorname{Spin}(8)$ is the direct sum of 4 copies of the standard 2-dimensional real representation of $S^{1}$. Therefore, for a suitable orientation on $S^{-}$, the relation in $H^{16}\left(S^{8} / / \mathrm{SU}(2), \mathbf{Z}\right)$ must be

$$
\left(\chi\left(S^{-}\right)+y^{2}\right)^{2}=0 .
$$

Since $M$ is an $S^{11}$-bundle over $S^{8} / / \mathrm{SU}(2)$, we have one more relation in $H^{12}(M, \mathbf{Z})$, saying that the Euler class of this $S^{11}$-bundle is zero. The $S^{11}$-bundle is $S\left(S^{-} \oplus V_{\mathbf{R}}\right)$, and so its Euler class is $\chi\left(S^{-} \oplus V_{\mathbf{R}}\right)=\chi\left(S^{-}\right) y$ in $H^{12}\left(S^{8} / / S^{1}, \mathbf{Z}\right)$. Thus $M$ has cohomology ring

$$
\begin{aligned}
H^{*}(M, \mathbf{Z}) & =\mathbf{Z}\left[y, \chi\left(S^{-}\right)\right] /\left(\left(\chi\left(S^{-}\right)+y^{2}\right)^{2}=0, \chi\left(S^{-}\right) y=0\right) \\
& =\mathbf{Z}\left[y, \chi\left(S^{-}\right)\right] /\left(\chi\left(S^{-}\right)^{2}+y^{4}=0, \chi\left(S^{-}\right) y=0\right) .
\end{aligned}
$$


This is the cohomology ring of $\mathbf{H P}^{4} \#-\mathbf{C a P}^{2}$, not of $\mathbf{H P}^{4} \# \mathbf{C a P} \mathbf{P}^{2}$. So the biquotient $M$ is diffeomorphic to $\mathbf{H P}^{4} \#-\mathbf{C a P}^{2}$.

The proof that $\mathbf{C P}^{8} \#-\mathbf{C a P}^{2}$ is diffeomorphic to a biquotient $M$ is completely analogous: it is the quotient of the $S^{9}$-bundle $S\left(S^{-} \oplus \mathbf{R}^{2}\right)$ over $S^{8}$ by a free $S^{1}$-action. Here $S^{1}$ acts on $S^{8}$ and on the vector bundle $S^{-}$by the homomorphism $S^{1} \cong \operatorname{Spin}(2) \subset \operatorname{Spin}(9)$, and on $\mathbf{R}^{2}$ by the standard real representation of $S^{1}$. Thus $M$ is a biquotient of the form $\left(\operatorname{Spin}(9) \times S^{9}\right) /\left(\operatorname{Spin}(8) \times S^{1}\right)$.

Finally, we come to the most surprising examples of biquotients, starting with $\mathbf{C P}^{n} \# \mathbf{C} \mathbf{P}^{n}$. The manifold $\mathbf{C P}^{n}$ has an orientation-reversing diffeomorphism from $n$ odd, and so $\mathbf{C P}^{n} \# \mathbf{C P} \mathbf{P}^{n}$ for $n$ odd is diffeomorphic to $\mathbf{C P}^{n} \#-\mathbf{C P}^{n}$. For $n$ even, we will show that $\mathbf{C P}^{n} \# \mathbf{C P}^{n}$ is diffeomorphic to a biquotient $\left(S^{3} \times S^{2 n-1}\right) /\left(S^{1}\right)^{2}$, for a free isometric action of $\left(S^{1}\right)^{2}$ on $S^{3} \times S^{2 n-1}$. The action is defined by the following homomorphism from $\left(S^{1}\right)^{2}$ to the maximal torus $\left(S^{1}\right)^{2} \times\left(S^{1}\right)^{n}$ of $\mathrm{SO}(4) \times \mathrm{SO}(2 n)$ :

$$
(x, y) \mapsto\left((x, y),\left(x y^{-1}, x y, \ldots, x y\right)\right) .
$$

It is straightforward to check that this action of $\left(S^{1}\right)^{2}$ on $S^{3} \times S^{2 n-1}$ is free. Let $M$ be the quotient manifold. We use that the action of $\left(S^{1}\right)^{2}$ on $S^{3}$ has cohomogeneity one, with trivial generic stabilizer group and stabilizers at the two special orbits equal to the two factors $S^{1}$. It follows that $M$ is the union of a 2-disc bundle over $S^{2 n-1} / S^{1}=\mathbf{C P}^{n-1}$ (corresponding to the action of the first factor $S^{1}$ ) and a 2-disc bundle over $S^{2 n-1} / S^{1}=\mathbf{C P}^{n-1}$ (corresponding to the second $S^{1}$ ) along their common boundary, which is a sphere $S^{2 n-1}$. So $M$ is the connected sum of two copies of $\mathbf{C P}^{n}$, with some orientations. To work out the orientations, it is convenient to compute the cohomology ring of $M$.

The quotient manifold $M$ fits into a fibration

$$
S^{3} \times S^{2 n-1} \rightarrow M \rightarrow\left(\mathrm{B} S^{1}\right)^{2} .
$$

Here $\left(\mathrm{B} S^{1}\right)^{2}$ has cohomology ring $\mathbf{Z}[u, v]$ with $u$ and $v$ in $H^{2}$. From the description of the $\left(S^{1}\right)^{2}$-action, we read off that the Euler classes of the $S^{3}$-bundle and $S^{2 n-1}$-bundle over $\left(\mathrm{B} S^{1}\right)^{2}$ are $u v$ and $(u-v)(u+$ $v)^{n-1}$. Since these form a regular sequence in the polynomial ring $H^{*}\left(\left(\mathrm{~B} S^{1}\right)^{2}, \mathbf{Z}\right)$, we have

$$
\begin{aligned}
H^{*}(M, \mathbf{Z}) & =\mathbf{Z}[u, v] /\left(u v=0,(u-v)(u+v)^{n-1}=0\right) \\
& =\mathbf{Z}[u, v] /\left(u v=0, u^{n}=v^{n}\right) .
\end{aligned}
$$


This is the cohomology ring of $\mathbf{C P}^{n} \# \mathbf{C} \mathbf{P}^{n}$, and (for $n$ even) not that of $\mathbf{C P}^{n} \#-\mathbf{C P}^{n}$. Therefore the biquotient $M$ is diffeomorphic to $\mathbf{C P}^{n} \# \mathbf{C P}^{n}$.

We next show that $\mathbf{H} \mathbf{P}^{n} \# \mathbf{H} \mathbf{P}^{n}$ is diffeomorphic to a biquotient. Imitating the construction for $\mathbf{C P}^{n} \# \mathbf{C} \mathbf{P}^{n}$ might suggest identifying $\mathbf{H P}^{n} \# \mathbf{H P}^{n}$ with a biquotient $\left(S^{7} \times S^{8 n-1}\right) / \mathrm{SU}(2)^{2}$. That works for $n$ odd, but not for $n$ even. For $n$ even, we have to consider a more general type of biquotient, $\left(\mathrm{Sp}(4) \times S^{4 n-1}\right) / \mathrm{SU}(2)^{3}$. Here the group $\mathrm{SU}(2)^{3}$ acts on $\mathrm{Sp}(4)$ by a homomorphism $\mathrm{SU}(2)^{3} \rightarrow \mathrm{Sp}(4)^{2}$. To define this, first let $V_{i}$ for $i=1,2,3$ denote the standard 2-dimensional complex representation of the $i$ th factor of SU(2). Then the homomorphism $\mathrm{SU}(2)^{3} \rightarrow \mathrm{Sp}(4)^{2}$ is defined by $\left(V_{1} \oplus V_{2}, V_{3} \oplus \mathbf{C}^{2}\right)$. Also, let $W_{12}: \mathrm{SU}(2)^{2} \rightarrow \mathrm{SO}(4)$ be the natural double covering, viewed as a 4-dimensional real representation of the first two copies of $\mathrm{SU}(2)$, and let $\left(V_{3}\right)_{\mathbf{R}}: \mathrm{SU}(2) \rightarrow \mathrm{SO}(4)$ be the natural real faithful representation of the third copy of $\mathrm{SU}(2)$. We define the action of $\mathrm{SU}(2)^{3}$ on $S^{4 n-1}$ by the homomorphism $\mathrm{SU}(2)^{3} \rightarrow \mathrm{SO}(8 n)$ defined by $\left(V_{3}\right)_{\mathbf{R}}^{\oplus n-1} \oplus W_{12}$.

The action of $\mathrm{SU}(2)^{3}$ on $\mathrm{Sp}(4)$ has cohomogeneity one, with trivial generic stabilizer group and with stabilizers at the two special orbits both isomorphic to $\mathrm{SU}(2)$, the first conjugate to the subgroup $\{(x, 1, x)\}$ in $\mathrm{SU}(2)^{3}$ and the second conjugate to $\{(1, x, x)\}$. These two subgroups act freely on $S^{4 n-1}$, and so $\mathrm{SU}(2)^{3}$ acts freely on $\mathrm{Sp}(4) \times S^{4 n-1}$. Let $M$ be the quotient manifold. Using the description of the $\mathrm{SU}(2)^{3}$ action on $\operatorname{Sp}(4)$, we see that $M$ is the union of two disc bundles over $S^{4 n-1} / \mathrm{SU}(2)=\mathbf{H P}^{n-1}$ along their common boundary, $S^{4 n-1}$. Therefore $M$ is the connected sum of two copies of $\mathbf{H P}^{n}$, with some orientations.

To determine the relevant orientations, it suffices for $n$ even to compute the cohomology ring of $M$. For $n$ odd, the manifolds $\mathbf{H P}^{n} \# \mathbf{H} \mathbf{P}^{n}$ and $\mathbf{H} \mathbf{P}^{n} \#-\mathbf{H P}^{n}$ have isomorphic cohomology rings. In that case we will also need a mod 3 Steenrod operation to see that $M$ is diffeomorphic to $\mathbf{H P}^{n} \# \mathbf{H} \mathbf{P}^{n}$ rather than $\mathbf{H P}^{n} \#-\mathbf{H P}^{n}$. First, we compute the cohomology ring of the homotopy quotient $\mathrm{Sp}(4) / / \mathrm{SU}(2)^{3}$, with respect to the above action of $\mathrm{SU}(2)^{3}$ on $\operatorname{Sp}(4)$. Write $z_{i}=-c_{2} V_{i}$, for $i=1,2,3$, which are generators in $H^{4}$ of the polynomial ring $H^{*}\left(\operatorname{BSU}(2)^{3}, \mathbf{Z}\right)$. The generators $c_{2}$ and $c_{4}$ of $H^{*}(\mathrm{BSp}(4), \mathbf{Z})$ pull back under the left homomorphism $\mathrm{SU}(2)^{3} \rightarrow \mathrm{Sp}(4)$ to $-z_{1}-z_{2}$ and $z_{1} z_{2}$, and under the right homomorphism $\mathrm{SU}(2)^{3} \rightarrow \mathrm{Sp}(4)$ to $-z_{3}$ and 0 . Therefore, using the Eilenberg-Moore spectral sequence as suggested by Singhof [25], the co- 
homology of $\mathrm{Sp}(4) / / \mathrm{SU}(2)^{3}$ is

$$
\mathbf{Z}\left[z_{1}, z_{2}, z_{3}\right] /\left(z_{1}+z_{2}=z_{3}, z_{1} z_{2}=0\right) .
$$

The manifold $M$ is an $S^{4 n-1}$-bundle over $\mathrm{Sp}(4) / / \mathrm{SU}(2)^{3}$, up to homotopy. The Euler class of this bundle, $\left(V_{3}\right)_{\mathbf{R}}^{\oplus n-1} \oplus W_{12}$, is $\pm\left(-z_{3}\right)^{n-1}\left(-z_{1}+\right.$ $z_{2}$ ), and so $M$ has cohomology ring

$$
\begin{aligned}
& \mathbf{Z}\left[z_{1}, z_{2}, z_{3}\right] /\left(z_{1}+z_{2}=z_{3}, z_{1} z_{2}=0, z_{3}^{n-1}\left(z_{1}-z_{2}\right)=0\right) \\
& =\mathbf{Z}\left[z_{1}, z_{2}\right] /\left(z_{1} z_{2}=0, z_{1}^{n}=z_{2}^{n}\right) .
\end{aligned}
$$

This is the cohomology ring of $\mathbf{H} \mathbf{P}^{n} \# \mathbf{H} \mathbf{P}^{n}$, and for $n$ even it is not the cohomology ring of $\mathbf{H P} \mathbf{P}^{n} \#-\mathbf{H} \mathbf{P}^{n}$. So $M$ is diffeomorphic to $\mathbf{H} \mathbf{P}^{n} \# \mathbf{H} \mathbf{P}^{n}$ for $n$ even. For $n$ odd, $n \geq 3$, we also need to observe that the classes $z_{i}$ are distinguished from their negatives by the fact that $P^{1} z_{i}=-z_{i}^{2}$ in $H^{8}\left(M, \mathbf{F}_{3}\right)$. By definition of the natural orientation on $\mathbf{H P}^{n}$ for $n \geq 2$, this means that $M$ is diffeomorphic to $\mathbf{H P}^{n} \# \mathbf{H} \mathbf{P}^{n}$ for all $n \geq 2$, not to $\mathbf{H P}^{n} \#-\mathbf{H P}^{n}$. The case $n=1$ is trivial, since $\mathbf{H P}^{n} \# \mathbf{H} \mathbf{P}^{n}=S^{4} \# S^{4}=$ $S^{4}$.

The last Cheeger manifolds which are diffeomorphic to biquotients are the manifolds $\mathbf{C} \mathbf{P}^{4 e+2} \# \mathbf{H} \mathbf{P}^{2 e+1}$. The relevant biquotient $M$ has the form $M=\left(S^{5} \times S^{8 e+3}\right) /\left(S^{1} \times \mathrm{SU}(2)\right)$. To be more explicit, let $L$ be the standard 1-dimensional complex representation of $S^{1}$, and let $V$ be the standard 2-dimensional complex representation of $\mathrm{SU}(2)$. Then we let $S^{1} \times \mathrm{SU}(2)$ act on $S^{5}$ as the unit sphere in $V \oplus L$, and on $S^{4 n-1}$ as the unit sphere in $\left(V \otimes_{\mathbf{C}} L\right)^{\oplus 2 e+1}$. The action of $S^{1} \times \mathrm{SU}(2)$ on $S^{5}$ has cohomogeneity one, with trivial generic stabilizer and with stabilizers at the two special orbits conjugate to the two factors $S^{1}$ and $\mathrm{SU}(2)$, respectively. Both of these subgroups act freely on $S^{8 e+3}$, and so $S^{1} \times \mathrm{SU}(2)$ acts freely on $S^{5} \times S^{8 e+3}$. Let $M$ be the quotient manifold.

From the action of $S^{1} \times \mathrm{SU}(2)$ on $S^{5}$, we see that $M$ is the union of a disc bundle over $S^{8 e+3} / S^{1}=\mathbf{C P}^{4 e+1}$ and a disc bundle over $S^{8 e+3} / \mathrm{SU}(2)=\mathbf{H P}^{2 e}$ along their common boundary, $S^{8 e+3}$. It follows that $M$ is the connected sum of $\mathbf{C P}^{4 e+2}$ and $\mathbf{H} \mathbf{P}^{2 e+1}$ with some orientations.

To show that $M$ is diffeomorphic to $\mathbf{C P}^{4 e+2} \# \mathbf{H} \mathbf{P}^{2 e+1}$ rather than to $\mathbf{C} \mathbf{P}^{4 e+2} \#-\mathbf{H P}^{2 e+1}$, we compute the cohomology ring and a mod 3 Steenrod operation on $M$. Since $M=S(V \oplus L) \times S\left((V \otimes L)^{\oplus 2 e+1}\right) /\left(S^{1} \times\right.$ $\mathrm{SU}(2))$, we can view $M$ as an $\left(S^{5} \times S^{8 e+3}\right)$-bundle over $\mathrm{B} S^{1} \times \mathrm{BSU}(2)$. Here $\mathrm{B} S^{1} \times \mathrm{BSU}(2)$ has cohomology ring $\mathbf{Z}[x, z]$, where we let $x=$ $c_{1} L$ and $z=-c_{2} V$. The vector bundles $V \oplus L$ and $(V \otimes L)^{\oplus 2 e+1}$ on 
$\mathrm{B} S^{1} \times \operatorname{BSU}(2)$ have Euler classes $(-z) x$ and $\left(x^{2}-z\right)^{2 e+1}$. So $M$ has cohomology ring

$$
\begin{aligned}
& \mathbf{Z}[x, z] /\left(-x z=0,\left(x^{2}-z\right)^{2 e+1}=0\right) \\
& =\mathbf{Z}[x, z] /\left(x z=0, x^{4 e+2}=z^{2 e+1}\right) .
\end{aligned}
$$

Here the element $z$ in $H^{4}(M, \mathbf{Z})$ is distinguished from its negative, for $e \geq 1$, by the property that $P^{1} z=-z^{2}$ in $H^{8}\left(M, \mathbf{F}_{3}\right)$. By definition of the natural orientation on $\mathbf{H P}^{2 e+1}$ for $e \geq 1, M$ is diffeomorphic to $\mathbf{C P}^{4 e+2} \# \mathbf{H} \mathbf{P}^{2 e+1}$, not to $\mathbf{C P}{ }^{4 e+2} \#-\mathbf{H P}^{2 e+1}$.

By contrast, the analogous calculation of the cohomology ring shows that the biquotient

$$
\left(S(V \oplus L) \times S\left((V \otimes L)^{\oplus 2 e}\right)\right) /\left(S^{1} \times \mathrm{SU}(2)\right)
$$

is diffeomorphic to $\mathbf{C} \mathbf{P}^{4 e} \#-\mathbf{H} \mathbf{P}^{2 e}$, not to $\mathbf{C P} \mathbf{P}^{4 e} \# \mathbf{H} \mathbf{P}^{2 e}$. This will be used in Section 8.

\section{Simplifying the description of a given biquotient manifold}

Here is an elementary but essential beginning to our simplification of the description of a given biquotient manifold. (Throughout the paper, $G$ and $H$ will denote compact Lie groups.)

Lemma 3.1. Let $M$ be a simply connected biquotient manifold. Then we can write $M=G / H$ for some simply connected group $G$ and connected group $H$ acting on $G$ by a homomorphism $H \rightarrow(G \times$ $G) / Z(G)$. If $M$ is 2-connected, then $H$ is simply connected and $H$ acts on $G$ by a homomorphism $H \rightarrow G \times G$.

Proof. Since $M$ is connected, we can write $M$ as a biquotient $G / H$ with $G$ connected. Since $M$ is simply connected, the long exact sequence of the fibration $H \rightarrow G \rightarrow M$,

$$
\pi_{1} H \rightarrow \pi_{1} G \rightarrow \pi_{1} M \rightarrow \pi_{0} H \rightarrow \pi_{0} G,
$$

shows that $H$ is connected and $\pi_{1} H \rightarrow \pi_{1} G$ is surjective. Let $C$ be the kernel of $\pi_{1} H \rightarrow \pi_{1} G$, a finitely generated abelian group.

Let $\widetilde{G}$ and $\widetilde{H}$ be the universal covers of $G$ and $H$. We can identify $\pi_{1} H$ with the kernel of $\widetilde{H} \rightarrow H$, and so $C$ is a central subgroup of $\widetilde{H}$. We have $\widetilde{G} \cong K_{G} \times \mathbf{R}^{a}$ and $\widetilde{H} \cong K_{H} \times \mathbf{R}^{b}$ for some simply connected compact Lie groups $K_{G}$ and $K_{H}$. The given homomorphism $H \rightarrow$ 
$(G \times G) / Z(G)$ lifts to a homomorphism $\widetilde{H} \rightarrow \widetilde{G} \times \widetilde{G}$. The resulting action of $\widetilde{H}$ on $\widetilde{G}$ is trivial on the subgroup $C$, and $\widetilde{H} / C$ acts freely on $\widetilde{G}$ with $M=\widetilde{G} /(\widetilde{H} / C)$.

Here $\widetilde{G}=K_{G} \times \mathbf{R}^{a}$, and the action of $\widetilde{H} / C$ on $\widetilde{G}$ is the product of an action on $K_{G}$ and an action on $\mathbf{R}^{a}$. Furthermore, since the group $\mathbf{R}^{a}$ is abelian, $\widetilde{H} / C$ acts on $\mathbf{R}^{a}$ by translations (left or right translations being the same). Since $\widetilde{H} / C$ is a connected group and the quotient is compact, $\widetilde{H} / C$ must act transitively on $\mathbf{R}^{a}$. Let $L$ be the kernel of the action of $\widetilde{H} / C$ on $\mathbf{R}^{a}$. Then $L$ must act freely on $K_{G}$, with $M=K_{G} / L$. Here $K_{G}$ is a simply connected compact Lie group. Also, $L$ must be connected by the long exact sequence

$$
\pi_{1} M \rightarrow \pi_{0} L \rightarrow \pi_{0} K_{G}
$$

Finally, if $M$ is 2-connected, then the same long exact sequence shows that $L$ is simply connected. In this case, the homomorphism $L \rightarrow\left(K_{G} \times K_{G}\right) / Z(G)$ lifts uniquely to a homomorphism $L \rightarrow K_{G} \times K_{G}$. q.e.d.

Since we intend to study simply connected biquotient manifolds, Lemma 3.1 tells us that we can assume $G$ is simply connected, and thus a product of simply connected simple groups. Thus, much of the complexity of more general compact Lie groups is avoided.

The main method of simplifying the description of a given biquotient is the following easy observation.

Lemma 3.2. Let $H$ be a compact Lie group acting on manifolds $X_{1}$ and $X_{2}$ such that $H$ acts transitively on $X_{1}$ and $H$ acts freely on $X_{1} \times X_{2}$. Let $K \subset H$ be the stabilizer of some point in $X_{1}$. Then the quotient manifold $\left(X_{1} \times X_{2}\right) / H$ is diffeomorphic to $X_{2} / K$, where $K$ acts freely on $X_{2}$ by the restriction of the action of $H$.

Proof. This is clear by identifying $X_{1}$ with $H / K$.

q.e.d.

Applying this method of simplification to biquotients gives the following fundamental result.

Lemma 3.3. Let $M$ be a simply connected biquotient manifold. Then we can write $M=G / H$ such that $G$ is simply connected, $H$ is connected, and $H$ does not act transitively on any simple factor of $G$.

Proof. By Lemma 3.1, we can write $M$ as a biquotient $G / H$ with $G$ simply connected and $H$ connected. If $H$ acts transitively on some simple factor of $G$, then Lemma 3.2 allows us to remove that factor of $G$ 
while replacing $H$ by a subgroup. The exact sequence $\pi_{1} M \rightarrow \pi_{0} H \rightarrow$ $\pi_{0} G$ shows that the new subgroup $H$ is still connected. By induction on the number of simple factors of $G$, we can arrange that $H$ does not act transitively on any simple factor of $G$.

Convention 3.4. From now on, we will only consider simply connected biquotient manifolds, and in writing $M=G / H$ we will assume that $G$ and $H$ satisfy the properties listed in Lemma 3.3 .

\section{Bounding $G$ in terms of $M$}

As mentioned in Convention 3.4, for the rest of the paper we only consider simply connected biquotient manifolds. We can and do assume that every such manifold $M$ is written $M=G / H$ with $G$ simply connected, $H$ connected, and $H$ not acting transitively on any simple factor of $G$. In this section, we will show how these properties determine $G$ up to finitely many possibilities in terms of the rational homotopy groups of $M$.

The following lemma will not be needed in this section, but is included here for later use.

Lemma 4.1. Let $M=G / H$ be a simply connected biquotient manifold. If in addition the rational homotopy group $\pi_{3} M_{\mathbf{Q}}$ is zero, then each simple factor $H_{1}$ of $H$ acts trivially on all factors of $G$ isomorphic to $H_{1}$.

Proof. By the long exact sequence of the fibration $H \rightarrow G \rightarrow M$, since $\pi_{3} M_{\mathbf{Q}}=0$, the homomorphism $\pi_{3} H_{\mathbf{Q}} \rightarrow \pi_{3} G_{\mathbf{Q}}$ is surjective. In particular, for each simple factor $G_{1}$ of $G, \pi_{3} H_{\mathbf{Q}} \rightarrow \pi_{3}\left(G_{1}\right)_{\mathbf{Q}}$ is surjective. Suppose that some simple factor $H_{1}$ of $H$ which is isomorphic to $G_{1}$ acts nontrivially on $G_{1}$; we will derive a contradiction. Here, a priori, $H_{1}$ can act by left and right translation on $G_{1}$. If $H_{1}$ acts nontrivially on only one side of $G_{1}$, thus by a nontrivial homomorphism $H_{1} \rightarrow G_{1}$, then this homomorphism must be an isomorphism, since $H_{1}$ is simple. In particular, $H_{1}$ acts transitively on $G_{1}$, which contradicts Convention 3.4. Therefore, $H_{1}$ must act nontrivially on both sides of $G_{1}$. Then the two homomorphisms from $H_{1}$ to $G_{1}$ must both be isomorphisms, using simplicity of $H_{1}$ again. We now use that any automorphism of a simply connected simple group $G_{1}$ acts trivially on $\pi_{3}\left(G_{1}\right) \cong \mathbf{Z}$, as follows from the proof of this isomorphism using the Killing form. The homomorphism $\pi_{3}\left(H_{1}\right)_{\mathbf{Q}} \rightarrow \pi_{3}\left(G_{1}\right)_{\mathbf{Q}}$ is the difference of the homomor- 
phisms given by the two homomorphisms $H_{1} \rightarrow G_{1}$, and so it is zero. Furthermore, since the two homomorphisms $H_{1} \rightarrow G_{1}$ both have only finite centralizer, there is no room for the rest of $H$ to act on $G_{1}$; in other words, $H$ acts on $G_{1}$ through a quotient group isogenous to $H_{1}$. It follows that the homomorphism $\pi_{3} H_{\mathbf{Q}} \rightarrow \pi_{3}\left(G_{1}\right)_{\mathbf{Q}}$ is zero, contradicting what we know from $\pi_{3} M_{\mathbf{Q}}=0$. Thus, each simple factor $H_{1}$ of $H$ must act trivially on simple factors of $G$ isomorphic to $H_{1}$. $\quad$ q.e.d.

To find more precise information on $G$, we need the classification of the simple compact Lie groups. In particular, we use that a simple group $G$ has rational homotopy groups concentrated in odd degrees $2 d-1$, and we call the numbers $d$ that occur the degrees of $G$. Particularly important for us is the maximal degree of $G$, which we call $d(G)$. It is also called the Coxeter number of $G$. The degrees of $G$ are well-known in many contexts: we can also say that $H^{*}(G, \mathbf{Q})$ is an exterior algebra with generators in degrees $2 d-1$ where $d$ runs over the degrees of $G$, or that the degrees of $G$ are the degrees of the generators of the ring of invariants of the Weyl group acting on its reflection representation. We tabulate the degrees of the simple groups here, following Bourbaki [5] or Gorbatsevich-Onishchik ([14], Table 1, p. 127). To avoid repetitions, one can assume that $A_{l}$ has $l \geq 1, B_{l}$ has $l \geq 3, C_{l}$ has $l \geq 2$, and $D_{l}$ has $l \geq 4$.

\section{Table 4.2}

$$
\begin{aligned}
A_{l} & : 2,3, \ldots, l+1 \\
B_{l} & : 2,4,6, \ldots, 2 l \\
C_{l} & : 2,4,6, \ldots, 2 l \\
D_{l} & : 2,4,6, \ldots, 2 l-2 ; l \\
G_{2} & : 2,6 \\
F_{4} & : 2,6,8,12 \\
E_{6} & : 2,5,6,8,9,12 \\
E_{7} & : 2,6,8,10,12,14,18 \\
E_{8} & : 2,8,12,14,18,20,24,30 .
\end{aligned}
$$

Using Table 4.2 and the known low-dimensional representations of each group, Onishchik proved the following result [20]. He later gave a more systematic proof, using reflection groups [21].

Lemma 4.3. Let $H \rightarrow G$ be a nontrivial homomorphism of simply connected simple groups. Then the maximal degrees satisfy $d(H) \leq$ 
$d(G)$. Moreover, if $d(H)=d(G)$, then either $H \rightarrow G$ is an isomorphism or $G / H$ is one of the following homogeneous spaces. On the right we show the degrees of $G$ and $H$.

$$
\begin{array}{lrl}
\operatorname{Spin}(2 n) / \operatorname{Spin}(2 n-1) & & \\
=S^{2 n-1}, n \geq 4 & 2,4,6, \ldots, 2 n-2 ; n & 2,4,6, \ldots, 2 n-2 \\
\mathrm{SU}(2 n) / \operatorname{Sp}(2 n), n \geq 2 & 2,3,4, \ldots, 2 n & 2,4,6, \ldots 2 n \\
\operatorname{Spin}(7) / G_{2}=S^{7} & 2,4,6 & 2,6 \\
\operatorname{Spin}(8) / G_{2}=S^{7} \times S^{7} & 2,4,4,6 & 2,6 \\
E_{6} / F_{4} & 2,5,6,8,9,12 & 2,6,8,12 .
\end{array}
$$

In all these cases except $\operatorname{Spin}(8) / \operatorname{Spin}(7)=S^{7}$, there is a unique conjugacy class of nontrivial homomorphisms $H \rightarrow G$; in the case $\operatorname{Spin}(8) / \operatorname{Spin}(7)$, there are three conjugacy classes which are equivalent under outer automorphisms of $\operatorname{Spin}(8)$. Also, in all the above cases, the centralizer of $H$ in $G$ is finite.

The following result shows how to apply Lemma 4.3 to biquotients $M=G / H$, although it is only a step on the way to the more precise Theorem 4.8. For a simple factor $G_{1}$ of $G$, we say that a degree $d$ of $G_{1}$ is killed by $H$ if the homomorphism $\pi_{2 d-1} H_{\mathbf{Q}} \rightarrow \pi_{2 d-1}\left(G_{1}\right)_{\mathbf{Q}}$ associated to the action of $H$ on $G_{1}$ is nonzero.

Lemma 4.4. Let $M=G / H$ be a simply connected biquotient, written according to Convention 3.4. Let $G_{1}$ be a simple factor of $G$ such that the maximal degree of $G_{1}$ is killed by $H$. Then either there is a simple factor $H_{1}$ of $H$ such that $H_{1}$ acts nontrivially on exactly one side of $G_{1}$ by one of the homomorphisms in Lemma 4.3 , so that $G_{1} / H_{1}$ is one of $\operatorname{Spin}(2 n) / \operatorname{Spin}(2 n-1)=S^{2 n-1}, \operatorname{SU}(2 n) / \operatorname{Sp}(2 n), \operatorname{Spin}(7) / G_{2}=S^{7}$, $\operatorname{Spin}(8) / G_{2}=S^{7} \times S^{7}$, or $E_{6} / F_{4} ;$ or $G_{1}$ is isomorphic to $\mathrm{SU}(2 n+1)$ for some $n$ and there is a simple factor $H_{1}$ of $H$ also isomorphic to $\mathrm{SU}(2 n+1)$ which acts on $G_{1}$ by $h(g)=h g h^{t}$. The $\mathrm{SU}(2 n+1)$ case cannot occur if $\pi_{3} M_{\mathbf{Q}}=0$.

Proof. We are given a simple factor $G_{1}$ of $G$ such that the maximal degree of $G_{1}$ is killed by $H$. It follows that there must be a simple factor $H_{1}$ of $H$ which kills the maximal degree $d$ of $G_{1}$. Since $H_{1}$ is simply connected, the action of $H_{1}$ on $G_{1}$ is given by a homomorphism $H_{1} \rightarrow$ $G_{1} \times G_{1}$, and the resulting linear map $\pi_{2 d-1}\left(H_{1}\right)_{\mathbf{Q}} \rightarrow \pi_{2 d-1}\left(G_{1}\right)_{\mathbf{Q}} \cong \mathbf{Q}$ is nonzero. This linear map is the difference of the two linear maps associated to the two homomorphisms $H_{1} \rightarrow G_{1}$ (on the left and right), so at least one of those two linear maps is nonzero. By Lemma 4.3, either $H_{1}$ is 
isomorphic to $G_{1}$ or $\left(G_{1}, H_{1}\right)$ is one of the pairs $(\operatorname{Spin}(2 n), \operatorname{Spin}(2 n-1))$, $(\mathrm{SU}(2 n), \operatorname{Sp}(2 n)),\left(\operatorname{Spin}(7), G_{2}\right),\left(\operatorname{Spin}(8), G_{2}\right)$, or $\left(E_{6}, F_{4}\right)$.

Suppose first that $\left(G_{1}, H_{1}\right)$ is one of these 5 pairs. If $H_{1}$ acts nontrivially on both sides of $G_{1}$, then in all cases except $(\operatorname{Spin}(8), \operatorname{Spin}(7))$, Lemma 4.3 implies that the two homomorphisms $H_{1} \rightarrow G_{1}$ are conjugate, so the resulting map $\pi_{*}\left(H_{1}\right)_{\mathbf{Q}} \rightarrow \pi_{*}\left(G_{1}\right)_{\mathbf{Q}}$ (the difference of the left and right maps) is 0 , contradicting the fact that $H_{1}$ kills the top degree of $G_{1}$. Even in the case (Spin(8), $\operatorname{Spin}(7)$ ), we compute that the outer automorphism group of $\operatorname{Spin}(8)$ acts trivially on the top degree, 6 , of $\operatorname{Spin}(8)$, that is, on $\pi_{11} \operatorname{Spin}(8)_{\mathbf{Q}}$. It follows that if $\operatorname{Spin}(7)$ acts nontrivially on both sides of Spin(8), then the left and right homomorphisms $\operatorname{Spin}(7) \rightarrow \operatorname{Spin}(8)$ give the same linear map into the top degree of Spin(8), and so the action of $\operatorname{Spin}(7)$ of $\operatorname{Spin}(8)$ cannot kill the top degree of $\operatorname{Spin}(8)$, a contradiction. Thus, in all these cases, $H_{1}$ acts nontrivially on only one side of $G_{1}$, by one of the homomorphisms in Lemma 4.3. The lemma is proved in this case.

The remaining case is where $H_{1}$ is isomorphic to $G_{1}$. Clearly this cannot occur if $\pi_{3} M_{\mathbf{Q}}=0$, by Lemma 4.1. In general, if $H_{1}$ acts nontrivially on only one side of $G_{1}$, then it must act by an isomorphism $H_{1} \rightarrow G_{1}$. So $H$ acts transitively on $G_{1}$, contrary to Convention 3.4. Therefore $H_{1}$ must act nontrivially on both sides of $G_{1}$, clearly by two isomorphisms $H_{1} \rightarrow G_{1}$. We are given that $H_{1}$ kills the top degree of $G_{1}$, so these two isomorphisms $H_{1} \rightarrow G_{1}$ must give different linear maps into the top degree of $\pi_{*}\left(G_{1}\right)_{\mathbf{Q}}$. So the outer automorphism group of $G_{1}$ must act nontrivially on the top degree of $G_{1}$. Of the simply connected simple groups, only $A_{n}, D_{n}$, and $E_{6}$ have nontrivial outer automorphism group, and only in the case $G_{1}=\mathrm{SU}(2 n+1)=A_{2 n}$ does the outer automorphism group act nontrivially on the top degree of $G_{1}$. (The outer automorphism group $\mathbf{Z} / 2$ of $\mathrm{SU}(n), n \geq 3$, acts by the identity on the even degrees of $\mathrm{SU}(n)$ and by -1 on the odd degrees. A topological way to see this is to identify $\pi_{*} G_{\mathbf{Q}}$ with the dual to the vector space $H^{>0}(\mathrm{~B} G, \mathbf{Q}) /\left(H^{>0} \cdot H^{>0}\right)$, and use in the case $G=\mathrm{SU}(n)$ that the outer automorphism $E \mapsto E^{*}$ acts on Chern classes by $c_{i} \mapsto(-1)^{i} c_{i}$.) So we must have $G_{1}=\mathrm{SU}(2 n+1)$, with $H_{1}=\mathrm{SU}(2 n+1)$ acting on $G_{1}$ by the identity on one side and by the outer automorphism $x \mapsto\left(x^{t}\right)^{-1}$ on the other.

q.e.d.

In order to have strong restrictions on the simple factors of $G$ in terms of the rational homotopy groups of $M=G / H$, we need to analyze more completely the way $H$ acts on simple factors of $G$ isomorphic to 
$\operatorname{Spin}(2 n), \operatorname{SU}(2 a), \operatorname{Spin}(7), \operatorname{Spin}(8)$, or $E_{6}$. In analogy with Lemmas 4.3 and 4.4, we will first formulate a general statement on the subgroups of these groups, and then apply it to biquotients. Before that, we need some simple topological results.

Lemma 4.5. Let $H$ and $G$ be connected compact Lie groups, and let $f: H \rightarrow G$ be any continuous map. If $f_{*}: \pi_{*} H_{\mathbf{Q}} \rightarrow \pi_{*} G_{\mathbf{Q}}$ is surjective, then $f$ is surjective.

Proof. Both $H$ and $G$ have the rational homotopy type of products of odd-dimensional spheres. So $H^{*}(H, \mathbf{Q})$ and $H^{*}(G, \mathbf{Q})$ are both exterior algebras, and the assumption means that the homomorphism

$$
f^{*}: H^{>0}(G, \mathbf{Q}) /\left(H^{>0} \cdot H^{>0}\right) \rightarrow H^{>0}(H, \mathbf{Q}) /\left(H^{>0} \cdot H^{>0}\right)
$$

is injective. Thus $H^{*}(H, \mathbf{Q})$ is the exterior algebra generated by the generators of $H^{*}(G, \mathbf{Q})$ together with some other generators. So $H^{*}(G, \mathbf{Q})$ $\rightarrow H^{*}(H, \mathbf{Q})$ is injective. Since $G$ is a closed orientable manifold, it follows that $H \rightarrow G$ is surjective.

q.e.d.

Corollary 4.6. Let $H$ and $G$ be connected compact Lie groups, with an action of $H$ on $G$ by a homomorphism $H \rightarrow(G \times G) / Z(G)$. If the associated homomorphism $\pi_{*} H_{\mathbf{Q}} \rightarrow \pi_{*} G_{\mathbf{Q}}$ is surjective, then $H$ acts transitively on $G$.

Proof. We are given that the homomorphism associated to the orbit map $f: H \rightarrow G$ of some point in $G$ is surjective on rational homotopy groups. By Lemma 4.5, $H \rightarrow G$ is surjective. That is, $H$ acts transitively on $G$.

q.e.d.

We now apply Corollary 4.6 to get information on the subgroups of the groups occurring in Lemma 4.3. (We also use the classification of simple Lie groups in the following proof, but it is more pleasant to use Corollary 4.6 when possible.)

Lemma 4.7. Let $\varphi: H \rightarrow \operatorname{Spin}(2 n)$ be a homomorphism from a simply connected simple group such that the homomorphism

$$
\pi_{2 n-1} H_{\mathbf{Q}} \rightarrow \pi_{2 n-1} \operatorname{Spin}(2 n)_{\mathbf{Q}} / \pi_{2 n-1} \operatorname{Spin}(2 n-1)_{\mathbf{Q}} \cong \mathbf{Q}
$$

is not zero. Then $H$ acts transitively on the sphere $\operatorname{Spin}(2 n) / \operatorname{Spin}(2 n-1)$ $=S^{2 n-1}$.

Next, let $G / K$ be one of the other homogeneous spaces from Lemma 4.3: $\mathrm{SU}(2 n) / \operatorname{Sp}(2 n)$ with $n \geq 2, \operatorname{Spin}(7) / G_{2}=S^{7}, \operatorname{Spin}(8) / G_{2}=$ 
$S^{7} \times S^{7}$, or $E_{6} / F_{4}$. Let $H \rightarrow G$ be a homomorphism from a simply connected compact Lie group $H$ which kills the second-largest degree of $G$. (In the case $G / K=\operatorname{Spin}(8) / G_{2}$, where $\operatorname{Spin}(8)$ has degrees $2,4,4,6$, we assume that both degrees 4 of $\operatorname{Spin}(8)$ are killed by $H$.) Then $H$ acts transitively on $G / K$.

Proof. In the cases where $G / K$ is one of $\operatorname{Spin}(2 n) / \operatorname{Spin}(2 n-1)=$ $S^{2 n-1}, \operatorname{Spin}(7) / G_{2}=S^{7}$, or $\operatorname{Spin}(8) / G_{2}=S^{7} \times S^{7}$, the assumption implies that the action of $H \times K$ on $G$ is surjective on rational homotopy groups. By Corollary 4.6, $H \times K$ acts transitively on $G$. Equivalently, $H$ acts transitively on $G / K$.

Next, let $G / K=\mathrm{SU}(2 n) / \mathrm{Sp}(2 n), n \geq 2$, and suppose that $H$ kills the second-largest degree, $2 n-1$, of $\mathrm{SU}(2 n)$. We can replace $H$ by one of its simple factors without changing this property. If $H$ is isomorphic to $G$, then the homomorphism $H \rightarrow G$ is an isomorphism, and so $H$ acts transitively on $G / K$. Otherwise, by Lemma $4.3, H$ has maximal degree at most that of $\mathrm{SU}(2 n)$, which is $2 n$, and if equality holds then $H=\operatorname{Sp}(2 n)$. But $\operatorname{Sp}(2 n)$ does not kill the degree $2 n-1$ of $\mathrm{SU}(2 n)$. So $H$ must have maximal degree $2 n-1$. By Table 4.2 , the only simple group with maximal degree an odd number is $H=\mathrm{SU}(2 n-1)$. Since $n \geq 2$, any nontrivial homomorphism $\mathrm{SU}(2 n-1) \rightarrow \mathrm{SU}(2 n)$ is equivalent to the standard inclusion by some automorphism of $\mathrm{SU}(2 n-1)$. Then $\mathrm{SU}(2 n-1)$ acts transitively on $\mathrm{SU}(2 n) / \mathrm{Sp}(2 n)$, because $\mathrm{Sp}(2 n)$ acts transitively on $\mathrm{SU}(2 n) / \mathrm{SU}(2 n-1)=S^{4 n-1}$.

Finally, suppose that $G / K=E_{6} / F_{4}$ and $H$ kills the second-largest degree, 9 , of $E_{6}$. We can replace $H$ by one of its simple factors without changing this property. Then $H$ has rank at most the rank 6 of $E_{6}$ and has 9 as a degree. By Table 4.2, it follows that $H$ is isomorphic to $E_{6}$. So the given homomorphism $H \rightarrow E_{6}$ must be an isomorphism, and in particular $H$ acts transitively on $E_{6} / F_{4}$. $\quad$ q.e.d.

We now apply this result on subgroups to deduce strong information on the classification of biquotient manifolds. For a given biquotient $M=$ $G / H$, we say that a given simple factor $G_{i}$ of $G$ contributes degree $d_{i}$ to $M$ if the homomorphism $\pi_{2 d_{i}-1} H_{\mathbf{Q}} \rightarrow \pi_{2 d_{i}-1}\left(G_{i}\right)_{\mathbf{Q}}$ is not surjective. If $G=\prod G_{i}$ and every factor $G_{i}$ contributes some degree $d_{i}$ to $M$, then $\pi_{2 d-1} M_{\mathbf{Q}}=\pi_{2 d-1} G_{\mathbf{Q}} / \pi_{2 d-1} H_{\mathbf{Q}}$ has dimension at least equal to the number of simple factors $G_{i}$ with $d_{i}=d$.

Theorem 4.8. Let $M=G / H$ be a simply connected biquotient manifold, written using Convention 3.4. Let $G_{1}$ be any simple factor of $G$. Then at least one of the following holds: 
(1) $G_{1}$ contributes its maximal degree to $M$.

(2) $G_{1}$ contributes its second-largest degree to $M$, and there is a simple factor $H_{1}$ of $G_{1}$ which acts nontrivially on exactly one side of $G_{1}$, with $G_{1} / H_{1}$ isomorphic to one of the homogeneous spaces $\mathrm{SU}(2 n) / \operatorname{Sp}(2 n)$ with $n \geq 2, \operatorname{Spin}(7) / G_{2}=S^{7}, \operatorname{Spin}(8) / G_{2}=S^{7} \times$ $S^{7}$, or $E_{6} / F_{4}$. The second-largest degree of $G_{1}$ is, respectively, $2 a-1,4,4$, or 9 . (In the case $G_{1}=\operatorname{Spin}(8)$, which has degrees $2,4,4,6$, the claim is only that $G_{1}$ contributes at least one degree 4 to $M$.

(3) $G_{1} \cong \operatorname{Spin}(2 n)$ with $n \geq 4$ contributes its degree $n$ to $M$, and there is a simple factor $H_{1} \cong \operatorname{Spin}(2 n-1)$ which acts nontrivially on exactly one side of $G_{1}$, by the standard inclusion, with $\operatorname{Spin}(2 n) / \operatorname{Spin}(2 n-1)=S^{2 n-1}$.

(4) $G_{1} \cong \mathrm{SU}(2 n+1)$ contributes degrees $2,4,6, \ldots, 2 n$ to $M$, and there is a simple factor $H_{1} \cong \mathrm{SU}(2 n+1)$ of $G_{1}$ which acts on $G_{1}$ by $h(g)=h g h^{t}$.

Proof. Let $G_{1}$ be a simple factor of $G$. Suppose that (1) does not hold, in other words that the maximal degree of $G_{1}$ is killed by $H$. By Lemma 4.4, there is a simple factor $H_{1}$ of $H$ such that either $H_{1}$ acts nontrivially on exactly one side of $G_{1}$ by one of the homomorphisms listed in (2) or (3) above, or $G_{1}=H_{1}=\mathrm{SU}(2 n+1)$ and $H_{1}$ acts on $G_{1}$ as in (4). The remaining point is to show that $G_{1}$ contributes the degrees to $M$ that we have claimed.

In cases (2) and (3), $H_{1}$ has finite centralizer in $G_{1}$ by Lemma 4.3, so the rest of $H$ can act on $G_{1}$ only on the other side from $H_{1}$. Since $H_{1}$ and the rest of $H$ together do not act transitively on $G_{1}$, by Convention 3.4, Lemma 4.7 shows that $G_{1}$ contributes its degree $n$ to $M$ if $G_{1} / H_{1}=$ $\operatorname{Spin}(2 n) / \operatorname{Spin}(2 n-1)=S^{2 n-1}$, or its second-largest degree to $M$ if $G_{1} / H_{1}$ is one of $\operatorname{SU}(2 n) / \operatorname{Sp}(2 n), \operatorname{Spin}(7) / G_{2}=S^{7}, \operatorname{Spin}(8) / G_{2}=S^{7} \times$ $S^{7}$, or $E_{6} / F_{4}$.

In case (4), since $H_{1}=\mathrm{SU}(2 n+1)$ acts with finite centralizer on both sides of $G_{1}=\mathrm{SU}(2 n+1)$, no other factor of $H$ can act on $G_{1}$. So the image of $\pi_{*} H_{\mathbf{Q}} \rightarrow \pi_{*}\left(G_{1}\right)_{\mathbf{Q}}$ is equal to the image of $\pi_{*}\left(H_{1}\right)_{\mathbf{Q}} \rightarrow$ $\pi_{*}\left(G_{1}\right)_{\mathbf{Q}}$. This homomorphism is the difference of the identity map on $\pi_{*}\left(G_{1}\right)_{\mathbf{Q}}$ with the map given by the outer automorphism $g \mapsto\left(g^{t}\right)^{-1}$, which acts by 1 on the even degrees and by -1 on the odd degrees (as shown in the proof of Lemma 4.4). So the image of $\pi_{2 d-1}\left(H_{1}\right)_{\mathbf{Q}} \rightarrow$ 
$\pi_{2 d-1}\left(G_{1}\right)_{\mathbf{Q}}$ is zero for $d$ even. That is, $G_{1}$ contributes all its even degrees $2,4, \ldots, 2 n$ to $M$. q.e.d.

Theorem 4.8 implies the following important qualitative statement on the classification of biquotients. The analogous result for homogeneous spaces is easy and probably well-known. It is perhaps surprising that the following statement requires the detailed classification work we have done in this section, but that seems to be true, at least for now.

Theorem 4.9. There are only finitely many diffeomorphism classes of 2-connected biquotient manifolds of a given dimension.

Theorem 4.9 is vaguely reminiscent of the Petrunin-Tuschmann theorem, which says in particular that for any number $C$, there are only finitely many diffeomorphism classes of 2-connected closed Riemannian manifolds with curvature $0 \leq K \leq C$ and diameter 1 [24]. But there is probably no way to actually deduce Theorem 4.9 from the PetruninTuschmann theorem, since there is no obvious upper bound on the curvature of 2-connected biquotients until one goes through the proof of Theorem 4.9. In fact, the discussion after Theorem 1.1 in my paper [27] shows that there can be no upper bound on the curvature of simply connected biquotients of dimension 6 , if one fixes their diameter to be 1.

Proof of Theorem 4.9. Any biquotient manifold $M$ is rationally elliptic; that is, all but finitely many of the rational homotopy groups of $M$ are zero. So, writing $n$ for the dimension of $M$, the odd-degree rational homotopy groups $\pi_{2 d-1} M_{\mathbf{Q}}$ are zero for $d>n$, and the total dimension of the odd-degree rational homotopy groups of $M$ is at most $n$, by Friedlander and Halperin ([13], p. 434). Since $M$ is simply connected, we can write $M$ as a biquotient $G / H$ according to Convention 3.4. In particular, $G$ is simply connected and, since $M$ is 2-connected, $H$ is also simply connected. The essential point is Theorem 4.8, which implies that each simple factor of $G$ contributes at least one degree to $M$, and that one such degree is at least half the maximal degree of $G$. Therefore the number of simple factors of $G$ is at most the total dimension of $\pi_{\text {odd }} M_{\mathbf{Q}}$ and hence at most $n$, and the maximal degree of each simple factor of $G$ is at most $2 n$. Thus $G$, being a product of simply connected simple groups, is determined up to finitely many possibilities by $n$. Also, $H$ is a simply connected group of dimension at most that of $G$, so $H$ and the homomorphism $H \rightarrow(G \times G) / Z(G)$ (up to conjugacy) are determined up to finitely many possibilities.

q.e.d. 


\section{Further general results on the classification of biquotients}

In this section we continue the previous section's method: we classify subgroups of compact Lie groups with certain properties, and apply the results to the classification of biquotients. The main result of this section is Theorem 5.3, which describes the possible simple factors of $G$ in a biquotient $M=G / H$ which contribute only their top degree to $M$. (This terminology is defined before Theorem 4.8.)

We begin by stating a classification of certain subgroups of $\operatorname{Spin}(2 n)$.

Lemma 5.1. Let $\varphi: H \rightarrow \operatorname{Spin}(2 n), n \geq 4$, be a homomorphism from a simply connected simple group $H$ such that the linear map

$$
\pi_{2 n-1} H_{\mathbf{Q}} \rightarrow \pi_{2 n-1} \operatorname{Spin}(2 n)_{\mathbf{Q}} / \pi_{2 n-1} \operatorname{Spin}(2 n-1)_{\mathbf{Q}} \cong \mathbf{Q}
$$

is not zero. Then $\varphi$ is either an isomorphism or one of the following homomorphisms, up to the standard $\mathbf{Z} / 2$ group of outer automorphisms of $\operatorname{Spin}(2 n): \mathrm{SU}(n) \hookrightarrow \operatorname{Spin}(2 n), \operatorname{Sp}(2 a) \hookrightarrow \operatorname{Spin}(4 a)$, the spin representation $\operatorname{Spin}(7) \hookrightarrow \operatorname{Spin}(8)$, or the spin representation $\operatorname{Spin}(9) \hookrightarrow \operatorname{Spin}(16)$.

This is straightforward to prove using the known degrees and lowdimensional representations of all the simple groups. Alternatively, we can deduce it from Borel's classification of groups which act linearly and transitively on the sphere, in the odd-dimensional case [4]. Namely, the hypothesis of Lemma 5.1 means that the action of $H$ on $S^{2 n-1}$ associated to the homomorphism $H \rightarrow \operatorname{Spin}(2 n)$ has orbit map $H \rightarrow$ $S^{2 n-1}$ such that $\pi_{2 n-1} H_{\mathbf{Q}} \rightarrow \pi_{2 n-1} S_{\mathbf{Q}}^{2 n-1} \cong \mathbf{Q}$ is not zero. It follows that $H \rightarrow S^{2 n-1}$ is surjective, in other words that $H$ acts transitively on $S^{2 n-1}$. Then Lemma 5.1 follows from Borel's classification.

The subgroups we classify next are the simple subgroups $H$ of any simple group $G$ such that the maximal degree of $H$ is at least the secondlargest degree of $G$. This is closely related to two classifications by Onishchik. First, he classified the simple subgroups $H \subset G$ such that $\operatorname{dim}_{\mathbf{Q}} \pi_{\text {odd }}(G / H)_{\mathbf{Q}}=1$ ([14], Table 3, p. 185), that is, $H$ kills all but one degree of $G$; these make up the first part of the list in Lemma 5.2. Next, he classified the simple subgroups $H \subset G$ such that $d(H) \geq d(G)-2$ ([22], Table 7.2, p. 195); it turns out that these include all the subgroups on the second part the list in Lemma 5.2.

Lemma 5.2. Let $H \rightarrow G$ be a nontrivial homomorphism of simply connected simple groups such that the maximal degree of $H$ is at least the second-largest degree of $G$ and is less than the maximal degree of $G$. Then $G / H$ is isomorphic to one of the following homogeneous spaces: 
On the left is the Dynkin index of the homomorphism $H \rightarrow G$. On the right are shown the degrees of $G$ not occurring in $H$, and the degrees of $H$ not occurring in $G$, in both cases with multiplicities. In the last column is the centralizer of $H$ in $G$, written modulo finite groups.

\begin{tabular}{|c|c|c|c|c|}
\hline & $\begin{array}{l}\mathrm{SU}(n) / \mathrm{SU}(n-1) \\
=S^{2 n-1}, n \geq 3\end{array}$ & $n$ & & $S^{1}$ \\
\hline 1 & $\operatorname{Sp}(2 n) / \operatorname{Sp}(2 n-2)$ & & & \\
\hline & $=S^{4 n-1}, n \geq 2$ & $2 n$ & & $A_{1}$ \\
\hline 1 & $\operatorname{Spin}(2 n+1) / \operatorname{Spin}(2 n)$ & & & \\
\hline & $=S^{2 n}, n \geq 3$ & $2 n$ & $n$ & 1 \\
\hline 1 & $\operatorname{Spin}(2 n+1) / \operatorname{Spin}(2 n-1)$ & & & \\
\hline & $=\mathrm{UT}\left(S^{2 n}\right), n \geq 3$ & $2 n$ & & $S^{1}$ \\
\hline 2 & $\mathrm{Sp}(4) / \mathrm{SU}(2)=\mathrm{UT}\left(S^{4}\right)$ & 4 & & $S^{1}$ \\
\hline 0 & $\mathrm{Sp}(4) / \mathrm{SU}(2)$ & 4 & & 1 \\
\hline 4 & $\mathrm{SU}(3) / \mathrm{SO}(3)$ & 3 & & 1 \\
\hline 1 & $\operatorname{Spin}(9) / \operatorname{Spin}(7)=S^{15}$ & 8 & & 1 \\
\hline 1 & $G_{2} / \mathrm{SU}(3)=S^{6}$ & 6 & 3 & 1 \\
\hline 1 & $G_{2} / \mathrm{SU}(2)=\mathrm{UT}\left(S^{6}\right)$ & 6 & & $A_{1}$ \\
\hline 3 & $G_{2} / \mathrm{SU}(2)$ & 6 & & $A_{1}$ \\
\hline 4 & $G_{2} / \mathrm{SO}(3)$ & 6 & & 1 \\
\hline 8 & $G_{2} / \mathrm{SO}(3)$ & 6 & & 1 \\
\hline 1 & $F_{4} / \operatorname{Spin}(9)=\mathbf{C a P}^{2}$ & 12 & 4 & 1 \\
\hline 1 & $\operatorname{Spin}(2 n) / \operatorname{Spin}(2 n-2)$ & & & \\
\hline & $=\mathrm{UT}\left(S^{2 n-1}\right), n \geq 4$ & $n, 2 n-2$ & $n-1$ & $S^{1}$ \\
\hline 1 & $\operatorname{Spin}(2 n) / \operatorname{Spin}(2 n-3), n \geq 4$ & $n, 2 n-2$ & & $A_{1}$ \\
\hline 1 & $\mathrm{SU}(2 n+1) / \mathrm{Sp}(2 n), n \geq 2$ & $3,5, \ldots, 2 n+1$ & & $S^{1}$ \\
\hline 2 & $\mathrm{SU}(2 n+1) / \mathrm{SO}(2 n+1), n \geq 2$ & $3,5, \ldots, 2 n+1$ & & 1 \\
\hline 1 & $\operatorname{Spin}(10) / \operatorname{Spin}(7)$ & 5,8 & & $S^{1}$ \\
\hline 2 & $\mathrm{SU}(7) / G_{2}$ & $3,4,5,7$ & & 1 \\
\hline 1 & $\operatorname{Spin}(9) / G_{2}$ & 4,8 & & $S^{1}$ \\
\hline 1 & $\operatorname{Spin}(10) / G_{2}$ & $4,5,8$ & & $A_{1}$. \\
\hline
\end{tabular}

The proof is straightforward for $G$ classical, using the known lowdimensional representations of each simple group, or alternatively the results by Onishchik mentioned above. For $G$ exceptional, more than enough information is provided by Dynkin's paper on the subgroups of the exceptional groups [8]. For example, Dynkin's Table 16 classifies the $A_{1}$ subgroups of $G_{2}$. Notice that the listings for $\operatorname{Spin}(9) / \operatorname{Spin}(7)=$ $S^{15}$ and $\operatorname{Spin}(10) / \operatorname{Spin}(7)$ refer to the spin representation of $\operatorname{Spin}(7)$; 
these spaces are different from the spaces $\operatorname{Spin}(2 n+1) / \operatorname{Spin}(2 n-1)=$ $\mathrm{UT}\left(S^{2 n}\right)$ for $n=4$ and $\operatorname{Spin}(2 n) / \operatorname{Spin}(2 n-3)$ for $n=5$. Also, there are three conjugacy classes of nontrivial homomorphisms $\mathrm{SU}(2) \rightarrow \mathrm{Sp}(4)$, where we write $V$ for the standard representation of $\mathrm{SU}(2): V \oplus \mathbf{C}^{2}$, where $\operatorname{Sp}(4) / \mathrm{SU}(2)=\operatorname{Sp}(4) / \mathrm{Sp}(2)=S^{7}$, which has Dynkin index 1; $V \oplus V$, where $\operatorname{Sp}(4) / \mathrm{SU}(2)=\operatorname{Spin}(5) / \operatorname{Spin}(3)=\mathrm{UT}\left(S^{4}\right)$, which has Dynkin index 2; and $S^{3} V$, which has Dynkin index 10 .

We now apply Lemma 5.2 to the classification of biquotients. Together with Theorem 4.8, the following theorem will be our most important tool in the classification of biquotients.

Theorem 5.3. Let $M=G / H$ be a simply connected biquotient, written according to Convention 3.4. Let $G_{1}$ be a simple factor of $G$ which contributes only its top degree to $M$. Then at least one of the following holds:

(1) $G_{1}$ is isomorphic to $\mathrm{SU}(2)$, which has degree 2.

(2) $G_{1}$ is a rank-2 group $\mathrm{SU}(3), \mathrm{Sp}(4)$, or $G_{2}$, with top degree $3,4,6$ respectively, and there is a simple factor $H_{1} \cong \mathrm{SU}(2)$ of $H$ which acts nontrivially on $G_{1}$.

(3) There is a simple factor $H_{1}$ of $H$ such that $H_{1}$ acts nontrivially on exactly one side of $G_{1}$ and $G_{1} / H_{1}$ is one of the homogeneous spaces in the first part of Lemma 5.2 's list.

(4) There are two simple factors $H_{1}$ and $H_{2}$ of $H$ which act nontrivially on the two sides of $G_{1}$ in one of the following ways, up to switching $H_{1}$ and $H_{2}: G_{1}=\operatorname{Spin}(2 n), n \geq 4, H_{1}$ is $\operatorname{Spin}(2 n-2)$ or $\operatorname{Spin}(2 n-3), H_{2}$ is $\mathrm{SU}(n)$, or $\operatorname{Sp}(2 a)$ with $n=2 a$, or $\operatorname{Spin}(9)$ with $n=8$; here $G_{1}$ has top degree $2 n-2$. Or $G_{1}=\mathrm{SU}(2 n+1)$, $n \geq 3, H_{1}$ is $\mathrm{Sp}(2 n)$ or $\mathrm{SO}(2 n+1)$, and $H_{2}=\mathrm{SU}(2 n-1)$; here $G_{1}$ has top degree $2 n+1$. Or $H_{1} \backslash G_{1} / H_{2}$ is one of $G_{2} \backslash \mathrm{SU}(7) / \mathrm{SU}(5)$, $G_{2} \backslash \operatorname{Spin}(9) / \mathrm{SU}(4), G_{2} \backslash \operatorname{Spin}(9) / \mathrm{Sp}(4)$, or $G_{2} \backslash \operatorname{Spin}(10) / \mathrm{SU}(5)$; here $G_{1}$ has top degree $7,8,8,8$, respectively.

Proof. If $G_{1}=\mathrm{SU}(2)$, we have conclusion (1). So we can assume that $G_{1}$ has rank at least 2. Equivalently, $G_{1}$ has at least two degrees. There must be a simple factor $H_{1}$ of $H$ which kills at least one secondlargest degree of $G_{1}$. (By Table 4.2, $G_{1}$ has a unique second-largest degree except when $G_{1}$ is $\operatorname{Spin}(8)$, which has degrees $2,4,4,6$.)

Suppose that $H_{1}$ is isomorphic to $G_{1}$. If $H_{1}$ acts nontrivially on only one side of $G_{1}$, then it acts by an isomorphism on that side of 
$G_{1}$, and so $H_{1}$ acts transitively on $G_{1}$, contrary to Convention 3.4. So $H_{1}$ must act by an isomorphism on both sides of $G_{1}$. Since all automorphisms of $G_{1}$ act as the identity on $\pi_{3} G_{1}=\mathbf{Z}$, the resulting homomorphism $\pi_{3}\left(H_{1}\right)_{\mathbf{Q}} \rightarrow \pi_{3}\left(G_{1}\right)_{\mathbf{Q}}$ is zero. Also, since $H_{1}$ is acting with finite centralizer on both sides of $G_{1}$, the rest of $H$ cannot act on $G_{1}$, and so the whole homomorphism $\pi_{3} H_{\mathbf{Q}} \rightarrow \pi_{3}\left(G_{1}\right)_{\mathbf{Q}}$ is zero. That is, $G_{1}$ contributes its degree 2 to $M$. Since $G_{1}$ is not isomorphic to $\mathrm{SU}(2)$, this contradicts our assumption that $G_{1}$ contributes only its top degree to $M$.

Thus $H_{1}$ is not isomorphic to $G_{1}$. We know that $d\left(H_{1}\right) \leq d\left(G_{1}\right)$ by Lemma 4.3. Suppose that $d\left(H_{1}\right)=d\left(G_{1}\right)$. By Lemma $4.3,\left(G_{1}, H_{1}\right)$ is one of the pairs $\left(\operatorname{Spin}(2 n), \operatorname{Spin}(2 n-1),(\mathrm{SU}(2 n), \operatorname{Sp}(2 n)),\left(\operatorname{Spin}(7), G_{2}\right)\right.$, $\left(\operatorname{Spin}(8), G_{2}\right)$, or $\left(E_{6}, F_{4}\right)$. In all these cases except $(\operatorname{Spin}(8), \operatorname{Spin}(7))$, there is a unique conjugacy class of nontrivial homomorphisms $H_{1} \rightarrow$ $G_{1}$; moreover, even in the case (Spin(8), Spin(7)), all nontrivial homomorphisms are equivalent under automorphisms of $\operatorname{Spin}(8)$ and so they all give the same homomorphism $\pi_{3} H_{1} \rightarrow \pi_{3} G_{1}$. Also, the centralizer of $H_{1}$ in $G_{1}$ is finite in all cases. So, in all cases, if $H_{1}$ acts nontrivially on both sides of $G_{1}$, then no other factor of $H$ acts on $G_{1}$, and the homomorphism $\pi_{3} H_{\mathbf{Q}} \rightarrow \pi_{3}\left(G_{1}\right)_{\mathbf{Q}}$ is zero. That is, $G_{1}$ contributes its degree 2 to $M$, contrary to our assumption that $G_{1}$ contributes only its top degree to $M$. Therefore $H_{1}$ must act only on one side of $G_{1}$. But that implies, in all these cases, that $H_{1}$ kills the top degree of $G_{1}$. This contradicts our assumption that $G_{1}$ contributes its top degree to $M$.

So we must have $d\left(H_{1}\right)<d\left(G_{1}\right)$. Since $H_{1}$ kills at least one secondlargest degree of $G_{1}, d\left(H_{1}\right)$ must be at least the second-largest degree of $G_{1}$. Therefore $\left(G_{1}, H_{1}\right)$ must be one of the pairs listed in Lemma 5.2. If $H_{1}$ is isomorphic to $\mathrm{SU}(2)$, then $G_{1}$ has rank 2 and we have conclusion (2). We can now assume that $H_{1}$ is not isomorphic to $\mathrm{SU}(2)$.

Next, we will show that $H_{1}$ acts nontrivially on only one side of $G_{1}$ in all the remaining cases. Suppose that $H_{1}$ acts nontrivially on both sides of $G_{1}$. By Lemma 5.2, the centralizer of $H_{1}$ on each side of $G_{1}$ is at most finite by $A_{1}$. So any simple factor of $H$ other than $H_{1}$ which acts nontrivially on $G_{1}$ is isomorphic to $\mathrm{SU}(2)$. Thus $H_{1}$ by itself must kill all the degrees of $G_{1}$ greater than 2 and less than the maximal degree $d\left(G_{1}\right)$. This is clearly impossible for the pairs $\left(G_{1}, H_{1}\right)$ on the second part of Lemma 5.2's list, since in these cases $G_{1}$ contains at least one degree in the interval $\left(2, d\left(G_{1}\right)\right)$ with greater multiplicity than $H_{1}$ does.

So $\left(G_{1}, H_{1}\right)$ is on the first part of Lemma 5.2's list. Since $H_{1}$ is not isomorphic to $\mathrm{SU}(2)$, the list shows that all nontrivial homomorphisms 
$H_{1} \rightarrow G_{1}$ have Dynkin index 1 . Since $H_{1}$ acts nontrivially on both sides of $G_{1}$, it follows that the associated homomorphism $\pi_{3}\left(H_{1}\right)_{\mathbf{Q}} \rightarrow$ $\pi_{3}\left(G_{1}\right)_{\mathbf{Q}}$ is zero. That is, $H_{1}$ does not kill the degree 2 of $G_{1}$. Since the whole group $H$ does kill the degree 2 of $G_{1}$, at least one of the two nontrivial homomorphisms $H_{1} \rightarrow G_{1}$ must have centralizer containing an $A_{1}$ subgroup. By the first part of Lemma 5.2's list, it follows that the pair $\left(G_{1}, H_{1}\right)$ is $(\operatorname{Sp}(2 n), \operatorname{Sp}(2 n-2))$ for some $n \geq 3$.

But for $\left(G_{1}, H_{1}\right)$ equal to $(\operatorname{Sp}(2 n), \operatorname{Sp}(2 n-2))$ with $n \geq 3$, there is a unique conjugacy class of nontrivial homomorphisms $H_{1} \rightarrow G_{1}$. Since $H_{1}$ acts nontrivially on both sides of $G_{1}$, it follows that the associated homomorphism $\pi_{*}\left(H_{1}\right)_{\mathbf{Q}} \rightarrow \pi_{*}\left(G_{1}\right)_{\mathbf{Q}}$ is zero. In particular, $H_{1}$ does not kill the second-largest degree, $2 n-2$, of $G_{1}$. This is a contradiction. We have thus completed the proof that $H_{1}$ acts nontrivially on only one side of $G_{1}$.

For spaces $G_{1} / H_{1}$ on the first part of Lemma 5.2's list, this completes the proof of conclusion (3). It remains to consider spaces $G_{1} / H_{1}$ on the second part of Lemma 5.2's list. To prove conclusion (4), we need to identify another simple factor of $H$ which acts on $G_{1}$.

In several cases on the second part of Lemma 5.2's list, $G_{1}$ is isomorphic to $\operatorname{Spin}(2 n), n \geq 4$. Here $H_{1}$ is either $\operatorname{Spin}(2 n-2)$, $\operatorname{Spin}(2 n-3)$, or, for $n=5, \operatorname{Spin}(7)$ (with a different homomorphism to $\operatorname{Spin}(10)$ ) or $G_{2}$. In all these cases, the degree $n$ of $G_{1}$ is not killed by $H_{1}$, meaning that $\pi_{2 n-1}\left(H_{1}\right)_{\mathbf{Q}} \rightarrow \pi_{2 n-1}\left(G_{1}\right)_{\mathbf{Q}}$ is not surjective. So there must be another simple factor $H_{2}$ of $H$ which acts on $G_{1}$ such that $\pi_{2 n-1}\left(H_{2}\right)_{\mathbf{Q}} \rightarrow \pi_{2 n-1}\left(G_{1}\right)_{\mathbf{Q}} / \pi_{2 n-1}\left(H_{1}\right)_{\mathbf{Q}}$ is not zero. If $n$ is odd, this just means that $\pi_{2 n-1}\left(H_{2}\right)_{\mathbf{Q}} \rightarrow \pi_{2 n-1}\left(G_{1}\right)_{\mathbf{Q}}$ is not zero. On the other hand, if $n$ is even, then we can always assume, after automorphisms of $H_{1}$ and $G_{1}$, that $H_{1} \rightarrow G_{1}$ is the standard inclusion. So, for $n$ odd or even, we can say that

$$
\pi_{2 n-1}\left(H_{2}\right)_{\mathbf{Q}} \rightarrow \pi_{2 n-1} \operatorname{Spin}(2 n)_{\mathbf{Q}} / \pi_{2 n-1} \operatorname{Spin}(2 n-1)_{\mathbf{Q}} \cong \mathbf{Q}
$$

is not zero. Here $H_{2}$ cannot be $\mathrm{SU}(2)$ since $n \geq 4$, so $H_{2}$ must act only on the other side of $G_{1}$ from $H_{1}$, since the centralizer of $H_{1}$ in $G_{1}$ is at most finite by $A_{1}$. By Lemma 5.1 , the space $G_{1} / H_{2}$ must be one of $\operatorname{Spin}(2 n) / \operatorname{SU}(n), \operatorname{Spin}(4 a) / \operatorname{Sp}(2 a), \operatorname{Spin}(8) / \operatorname{Spin}(7)$, or $\operatorname{Spin}(16) / \operatorname{Spin}(9)$. If $G_{1} / H_{2}=\operatorname{Spin}(8) / \operatorname{Spin}(7)$, then we can replace $H_{1}$ by $H_{2}$ and we have conclusion (3); so we can exclude that case. We have thus proved conclusion (4) for $G_{1}=\operatorname{Spin}(2 n)$.

Next, there are several cases in the second part of Lemma 5.2's list where $G_{1}=\mathrm{SU}(2 n+1), n \geq 2$. Here $H_{1}$ is either $\operatorname{Sp}(2 n)$, $\mathrm{SO}(2 n+1)$, 
or, for $n=3, G_{2}$. In all these cases, $H_{1}$ has only even degrees, and in particular it does not kill the degree $2 n-1$ of $G_{1}$. So there must be another simple factor $H_{2}$ of $H$ which kills the degree $2 n-1$ of $G_{1}$. Here $H_{2}$ must act nontrivially only on the other side of $G_{1}$ from $H_{1}$, because the centralizer of $H_{1}$ in $G_{1}$ is at most $S^{1}$ by finite in these cases. It is easy to read from Table 4.2 that any simple group $H_{2}$ which maps nontrivially to $G_{1}=\mathrm{SU}(2 n+1), n \geq 2$, and has $2 n-1$ as a degree is either $\mathrm{SU}(2 n-1), \mathrm{SU}(2 n)$, or $\mathrm{SU}(2 n+1)$. Here $H_{2}=\mathrm{SU}(2 n+1)$ is excluded by Convention 3.4, which says that $H$ does not act transitively on $G_{1}$. If $H_{2}=\mathrm{SU}(2 n)$, then we can replace $H_{1}$ by $H_{2}$ and we have conclusion (3). The remaining possibility is $H_{2}=\mathrm{SU}(2 n-1)$. We have proved conclusion (4) for $G_{1}=\mathrm{SU}(2 n+1)$.

The last case, from the second part of Lemma 5.2's list, is where $G_{1}$ is $\operatorname{Spin}(9)$ and $H_{1}$ is the exceptional group $G_{2}$. Here the degree 4 of $G_{1}$ is not killed by $H_{1}$, so it must be killed by some other simple factor $H_{2}$ of $H$. The centralizer of $H_{1}$ in $G_{1}$ is finite by $S^{1}$, so $H_{2}$ must act nontrivially only on the other side of $G_{1}$ from $H_{1}$. From Table 4.2, since $H_{2}$ has a degree 4 and maps nontrivially to $\operatorname{Spin}(9), H_{2}$ is one of $\operatorname{Sp}(4)$, $\mathrm{SU}(4), \operatorname{Spin}(7), \operatorname{Spin}(8)$, or $\operatorname{Spin}(9)$. The case $H_{2}=\operatorname{Spin}(9)$ is excluded by Convention 3.4, which says that $H$ does not act transitively on $G_{1}$. If $H_{2}$ is $\operatorname{Spin}(7)$ or $\operatorname{Spin}(8)$, then we can replace $H_{1}$ by $H_{2}$ and we have conclusion (3). The remaining possibilities for $\mathrm{H}_{2}$ are $\mathrm{Sp}(4)$ and $\mathrm{SU}(4)$. We have proved conclusion (4) for $G_{1}=\operatorname{Spin}(9)$.

q.e.d.

\section{Biquotients which are rational homology spheres}

As an application of Theorems 4.8 and 5.3, we now classify all biquotients which are simply connected rational homology spheres. The result seems surprising. In particular, the Gromoll-Meyer exotic 7-sphere which is a biquotient [15] is the only such example in any dimension. As mentioned in the introduction, Theorem 6.1 was found at the same time by Kapovitch and Ziller [17].

Theorem 6.1. Any biquotient which is a simply connected rational homology sphere is either a homogeneous manifold, the GromollMeyer exotic 7-sphere which is a biquotient $\mathrm{Sp}(4) / \mathrm{SU}(2)$, or a certain 4-connected 11-manifold with the integral homology groups of $\operatorname{UT}\left(S^{6}\right)$ which is a biquotient $G_{2} / \mathrm{SU}(2)$.

The homogeneous manifolds which are simply connected rational homology spheres are the sphere $S^{n}$, the unit tangent bundle $\mathrm{UT}\left(S^{2 n}\right)$, the 
Wu 5-manifold $\mathrm{SU}(3) / \mathrm{SO}(3)$ [7], the Berger 7-manifold $\mathrm{Sp}(4) / \mathrm{SU}(2)$ with $\pi_{3} M$ isomorphic to $\mathbf{Z} / 10$ [3], the 11-manifold $G_{2} / \mathrm{SU}(2)$ with $\pi_{3} M$ isomorphic to $\mathbf{Z} / 3$, and the two 11-manifolds $G_{2} / \mathrm{SO}(3)$ with $\pi_{3} M$ isomorphic to $\mathbf{Z} / 4$ or $\mathbf{Z} / 28$.

The homogeneous spaces in Theorem 6.1 were classified by Onishchik ([14], Table 3, p. 185). The nontrivial biquotient $G_{2} / \mathrm{SU}(2)$ was first discovered by Eschenburg ([10], pp. 166-170). Kapovitch and Ziller have shown that this biquotient $G_{2} / \mathrm{SU}(2)$ is diffeomorphic to the connected sum of $\mathrm{UT}\left(S^{6}\right)$ with some homotopy 11-sphere [17]. It is not known whether $G_{2} / \mathrm{SU}(2)$ is actually diffeomorphic to $\mathrm{UT}\left(S^{6}\right)$; one hopes for a negative answer, which would be more interesting.

Before starting the proof of Theorem 6.1 , we assemble some elementary facts about biquotients $\operatorname{Sp}(4) / H$. In the following lemma, we consider free actions of a group $H$ on $\operatorname{Sp}(4)$ given by a homomorphism $H \rightarrow \mathrm{Sp}(4)^{2} / Z(\mathrm{Sp}(4))$.

\section{Lemma 6.2.}

(1) There is no free action of $\mathrm{SO}(3)$ on $\mathrm{Sp}(4)$. Any free action of $\mathrm{SU}(2)$ on $\mathrm{Sp}(4)$ is either trivial on one side of $\mathrm{Sp}(4)$, so that $\mathrm{Sp}(4) / \mathrm{SU}(2)$ is $S^{7}, \mathrm{UT}\left(S^{4}\right)$, or the Berger 7 -manifold, or given by the homomorphisms $\left(V \oplus \mathbf{C}^{2}, V^{\oplus 2}\right)$ up to switching the two sides of $\mathrm{Sp}(4)$, so that $\mathrm{Sp}(4) / \mathrm{SU}(2)$ is the Gromoll-Meyer exotic 7-sphere [15]. Here $V$ denotes the standard representation of $\mathrm{SU}(2)$.

(2) Any free action of $\mathrm{SU}(2)^{2}$ on $\mathrm{Sp}(4)$ is given, up to switching the two $\mathrm{SU}(2)$ factors and switching the two sides of $\mathrm{Sp}(4)$, by the homomorphisms $\left(V_{1} \oplus V_{2}, \mathbf{C}^{4}\right)$ or $\left(V_{1} \oplus \mathbf{C}^{2}, V_{2}^{\oplus 2}\right)$. Here $V_{1}$ and $V_{2}$ are the standard representations of the two $\mathrm{SU}(2)$ factors. In both cases, the quotient $\mathrm{Sp}(4) / \mathrm{SU}(2)^{2}$ is diffeomorphic to $S^{4}$.

Proof. We only prove (1) here, the calculation for (2) being similar. There are three conjugacy classes of nontrivial homomorphisms $\mathrm{SU}(2) \rightarrow \mathrm{Sp}(4), V \oplus \mathbf{C}^{2}, V^{\oplus 2}$, and $S^{3} V$, by Lemma 5.2. If $\mathrm{SU}(2)$ acts trivially on one side of $\mathrm{Sp}(4)$, then $\mathrm{Sp}(4) / \mathrm{SU}(2)$ is one of the three homogeneous spaces mentioned in (1). So we can assume that $\mathrm{SU}(2)$ acts nontrivially on both sides of $\mathrm{Sp}(4)$. Let $H$ be $\mathrm{SU}(2)$ or $\mathrm{SU}(2) /\{ \pm 1\} \cong \mathrm{SO}(3)$. The group $H$ acts freely on $\mathrm{Sp}(4)$ if and only if the two images of each nontrivial element of $H$ in $\mathrm{Sp}(4)$ are not conjugate. In particular, the two homomorphisms $\mathrm{SU}(2) \rightarrow \mathrm{Sp}(4)$ must be non-conjugate. If one homomorphism is $V \oplus \mathbf{C}^{2}$ and the other is 
$V^{\oplus 2}$, then Gromoll and Meyer showed that $\mathrm{SU}(2)$ acts freely on $\mathrm{Sp}(4)$ and that the quotient manifold is an exotic 7-sphere [15]. Otherwise, $\mathrm{SU}(2)$ must act by $S^{3} V$ on one side and either $V \oplus \mathbf{C}^{2}$ or $V^{\oplus 2}$ on the other. Then neither $\mathrm{SU}(2)$ nor $\mathrm{SU}(2) /\{ \pm 1\}$ acts freely on $\mathrm{Sp}(4)$, since the images of the diagonal matrix $\left(\zeta_{3}, \zeta_{3}^{-1}\right)$ in $\mathrm{SU}(2)$ under $S^{3} V$ and $V \oplus \mathbf{C}^{2}$ are conjugate, and likewise the images of the diagonal matrix $\left(\zeta_{4}, \zeta_{4}^{-1}\right)$ in $\mathrm{SU}(2)$ under $S^{3} V$ and $V^{\oplus 2}$ are conjugate. (Here $\zeta_{n}$ denotes a primitive $n$th root of unity.) This proves (1).

Proof of Theorem 6.1. We write $M=G / H$ according to Convention 3.4. Since $M$ is a rational homology sphere, the odd-dimensional rational homotopy of $M$ has dimension 1 . In more detail, $S^{2 n-1}$ has 1dimensional rational homotopy in dimension $2 n-1$ and zero otherwise, while $S^{2 n}$ has 1-dimensional rational homotopy in dimensions $2 n$ and $4 n-1$, and zero otherwise. Since each simple factor of $G$ contributes at least dimension 1 to $\pi_{\text {odd }} M_{\mathbf{Q}}$ by Theorem $4.8, G$ must be simple. This already makes the situation much more understandable; it would not be clear at all without the analysis leading to Theorem 4.8.

If the simply connected rational homology sphere $M$ has dimension $r$ at most 4 , then it is automatically a homotopy sphere. It is therefore not surprising to find that $M$ is diffeomorphic to $S^{r}$ for $r \leq 4$. First, for $r=2$ we can just use that every homotopy 2-sphere is diffeomorphic to $S^{2}$. For $r \geq 3$, we have $\pi_{2} M_{\mathbf{Q}}=0$, and so $H$ has finite fundamental group; equivalently, $H$ is semisimple. For $r=3, \pi_{3} M \cong \mathbf{Z}$ and $\pi_{4} M_{\mathbf{Q}}=$ 0 , which implies that $G$ has one more simple factor than $H$ does. Since $G$ is simple, $H=1$. Since $M$ is a homotopy 3 -sphere, $G$ has degree 2 only, and so $G$ is $\mathrm{SU}(2)$. Thus $M$ is diffeomorphic to $\mathrm{SU}(2)$, that is, to $S^{3}$.

For $r=4, \pi_{2} M=\pi_{3} M=0$ and $\pi_{4} M \cong \mathbf{Z}$, which implies that $H$ is simply connected and has one more simple factor than $G$ does. Since $G$ is simple, $H$ has two simple factors. By the rational homotopy groups of $M, G$ must contribute degree 4 to $M$ while $H$ contributes degree 2 , and nothing else. By Theorems 4.8 and 5.3, there is a simple factor $H_{1}$ of $H$ such that either $G / H_{1}$ is a homogeneous space diffeomorphic to $S^{7}$, or $G / H_{1}$ is $\operatorname{Spin}(8) / G_{2}=S^{7} \times S^{7}$, or $\left(G, H_{1}\right)$ is $(\operatorname{Sp}(4), \mathrm{SU}(2))$. Let $H_{2}$ denote the other simple factor of $H$. If $G / H_{1}=S^{7}$, then $H_{2}$ has degree 2 only, so $\mathrm{H}_{2}$ is isomorphic to $\mathrm{SU}(2)$. In this case $M$ is the quotient of $S^{7}$ by $\mathrm{SU}(2)$ acting freely by a homomorphism $\mathrm{SU}(2) \rightarrow \mathrm{O}(8)$. Such a homomorphism is unique up to conjugacy, and so $M$ is the standard quotient $S^{4}$. Next, if $G / H_{1}$ is $\operatorname{Spin}(8) / G_{2}=S^{7} \times S^{7}$, then $H_{2}$ has degrees 
2 and 4 , and so $\mathrm{H}_{2}$ is isomorphic to $\mathrm{Sp}(4)$. But there is in fact no free isometric action of $\operatorname{Sp}(4)$ on $S^{7} \times S^{7}$, because the restriction of any action of $\mathrm{Sp}(4)$ on $S^{7}$ to the subgroup $\mathrm{SU}(2)=\mathrm{Sp}(2) \subset \mathrm{Sp}(4)$ has a fixed point. (Either the associated 8-dimensional complex representation of $\mathrm{Sp}(4)$ has a trivial summand, or it is the sum of two copies of the standard representation of $\mathrm{Sp}(4)$ and hence restricts on $\mathrm{SU}(2)$ to $V^{\oplus 2} \oplus \mathbf{C}^{4}$.)

For $r=4$, it remains to consider the case $\left(G, H_{1}\right)=(\operatorname{Sp}(4), \mathrm{SU}(2))$. Here $\mathrm{H}_{2}$ has degree 2 only, and so $\mathrm{H}_{2}$ is isomorphic to $\mathrm{SU}(2)$. Thus $M$ is a biquotient $\mathrm{Sp}(4) / \mathrm{SU}(2)^{2}$. By Lemma $6.2, M$ is diffeomorphic to $S^{4}$.

We proceed to the case $r \geq 5$. As mentioned earlier, we have $\pi_{2} M_{\mathbf{Q}}=0$, and so $H$ is semisimple. Since $\pi_{3} M_{\mathbf{Q}}=\pi_{4} M_{\mathbf{Q}}=0, H$ has the same number of simple factors as $G$. Since $G$ is simple, so is $H$.

One of the cases (1) to (4) in Theorem 4.8 must hold. Here (4) is excluded since $G$ is simple and $H$ acts freely on $G$. In case (3), $M$ is the homogeneous space $\operatorname{Spin}(2 n) / \operatorname{Spin}(2 n-1)=S^{2 n-1}$. In case (2), $M$ is one of the homogeneous spaces $\mathrm{SU}(2 a) / \mathrm{Sp}(2 a)$ with $a \geq 2$, $\operatorname{Spin}(7) / G_{2}=S^{7}, \operatorname{Spin}(8) / G_{2}=S^{7} \times S^{7}$, or $E_{6} / F_{4}$. Since $M$ is a rational homology sphere, considering the degrees of these homogeneous spaces shows that $M$ is either $\mathrm{SU}(4) / \mathrm{Sp}(4)=\operatorname{Spin}(6) / \operatorname{Spin}(5)=S^{5}$ or $\operatorname{Spin}(7) / G_{2}=S^{7}$.

There remains case (1) of Theorem 4.8, where $G$ contributes its top degree to $M$. In this case, one of conclusions (1) to (4) in Theorem 5.3 must hold. Here case (4) of Theorem 5.3 is excluded since $H$ is simple. In case (3), $M$ is one of the homogeneous spaces listed in the first part of Lemma 5.2. Since $M$ is a rational homology sphere, the possibilities are as listed in Theorem 6.1.

Case (1), $G=\mathrm{SU}(2)$, of Theorem 5.3 is excluded because we are considering biquotients $M$ of dimension $r \geq 5$. So there remains only case (2). That is, $H_{1}$ is $\mathrm{SU}(2), H$ is either $H_{1}=\mathrm{SU}(2)$ or $H_{1} /\{ \pm 1\} \cong \mathrm{SO}(3)$, and $M$ is a biquotient $\mathrm{SU}(3) / H, \operatorname{Sp}(4) / H$, or $G_{2} / H$, of dimension 5 , 7 , or 11 , respectively. The homogeneous spaces of this type are listed in Lemma 5.2. So we can assume that $H_{1}$ acts nontrivially on both sides of $G$. Biquotients of this type (a rank-2 group divided by a rank-1 group) were classified by Eschenburg ([10], pp. 166-170), but I will give my own proof since Eschenburg's paper is not widely available.

First, suppose $G=\mathrm{SU}(3)$. Then there are two conjugacy classes of nontrivial homomorphisms $\mathrm{SU}(2) \rightarrow \mathrm{SU}(3), V \oplus \mathbf{C}$ and $S^{2} V$, where $V$ denotes the standard representation of $\mathrm{SU}(2)$. Since $H$ (which is $\mathrm{SU}(2)$ or $\mathrm{SO}(3))$ acts freely on $G$, the two images in $\mathrm{SU}(3)$ of any nontrivial element of $H$ are not conjugate in $\mathrm{SU}(3)$. So SU(2) must act by $V \oplus \mathbf{C}$ 
on one side of $\mathrm{SU}(3)$ and by $S^{2} V$ on the other. But, if we write $\zeta_{a}$ for a primitive $a$ th root of unity, the diagonal matrix $\left(\zeta_{3}, \zeta_{3}^{-1}\right)$ in $\mathrm{SU}(2)$ has images under both homomorphisms to $\mathrm{SU}(3)$ which are conjugate to $\left(\zeta_{3}, 1, \zeta_{3}^{-1}\right)$. So neither $\mathrm{SU}(2)$ nor $\mathrm{SU}(2) /\{ \pm 1\}$ can be acting freely. Thus, the case $G=\mathrm{SU}(3)$ does not occur.

The case $G=\operatorname{Sp}(4)$ is covered by Lemma 6.2. Since $\mathrm{SU}(2)$ acts nontrivially on both sides of $\operatorname{Sp}(4), M$ is the Gromoll-Meyer exotic 7sphere.

The last case to consider is where $G$ is the exceptional group $G_{2}$. Here, as described in Lemma 5.2, there are 4 conjugacy classes of nontrivial homomorphisms SU(2) $\rightarrow G_{2}$, which we identify by their Dynkin index, 1,3 , 4, or 28. Using these Dynkin indices, we compute that the composed representation $\mathrm{SU}(2) \rightarrow G_{2} \rightarrow \mathrm{U}(7)$ must be: $W_{1}=V^{\oplus 2} \oplus \mathbf{C}^{3}$, $W_{3}=S^{2} V \oplus V^{\oplus 2}, W_{4}=\left(S^{2} V\right)^{\oplus 2} \oplus \mathbf{C}$, and $W_{28}=S^{6} V$. In particular, the homomorphisms $W_{4}$ and $W_{28}$ from $\mathrm{SU}(2)$ to $G_{2}$ are trivial on $\{ \pm 1\}$ in $\mathrm{SU}(2)$, and the other two are not.

We are assuming that $\mathrm{SU}(2)$ acts nontrivially on both sides of $G_{2}$. Since the action is free for either $\mathrm{SU}(2)$ or $\mathrm{SU}(2) /\{ \pm 1\}$, the two homomorphisms $\mathrm{SU}(2) \rightarrow G_{2}$ must be non-conjugate. There are now 6 cases to consider, corresponding to the 6 unordered pairs of distinct homomorphisms $W_{i}$. Notice that the action of $\mathrm{SU}(2)$ on $G_{2}$ is trivial on $\{ \pm 1\}$ if and only if the two homomorphisms send -1 to the same element of the center of $G_{2}$. The center of $G_{2}$ is trivial, so the two homomorphisms must both be trivial on -1 . That is, $\mathrm{SU}(2)$ acts on $G_{2}$ through its quotient $\mathrm{SO}(3)$ if and only if the two homomorphisms are $\left(W_{4}, W_{28}\right)$.

Given that $\mathrm{SU}(2)$ acts on $G_{2}$ by $W_{i}$ on one side and $W_{j}$ on the other, the action of $\mathrm{SU}(2)$ is free if and only if every nontrivial element of $\mathrm{SU}(2)$ has non-conjugate images in $G_{2}$ under the two homomorphisms. Every element of $\mathrm{SU}(2)$ is conjugate to a diagonal element $\left(x, x^{-1}\right)$ with $x \in S^{1}$, so it suffices to consider those elements. Furthermore, it is convenient to observe that two elements of $G_{2}$ are conjugate if and only if their images in $\mathrm{U}(7)$ are conjugate.

Using this, we can check whether each pair $\left(W_{i}, W_{j}\right)$ of homomorphisms $\mathrm{SU}(2) \rightarrow G_{2}$ gives a free action of $\mathrm{SU}(2)$ on $G_{2}$ or not. Here the image of $x \in S^{1} \subset \mathrm{SU}(2)$ in $\mathrm{U}(7)$ under the homomorphism $W_{i}$ is 
conjugate to the diagonal matrix:

$$
\begin{aligned}
W_{1} & :\left(x, x^{-1}, x, x^{-1}, 1,1,1\right) \\
W_{3} & :\left(x, x^{-1}, x, x^{-1}, x^{2}, 1, x^{-2}\right) \\
W_{4} & :\left(x^{2}, x^{-2}, x^{2}, x^{-2}, 1,1,1\right) \\
W_{28} & :\left(x^{-6}, x^{-4}, x^{-2}, 1, x^{2}, x^{4}, x^{6}\right) .
\end{aligned}
$$

The result is that 5 of the 6 unordered pairs $\left(W_{i}, W_{j}\right)$ do not give free actions of $\mathrm{SU}(2)$ (or $\mathrm{SO}(3)$, in the case $\left(W_{4}, W_{28}\right)$ ) on $G_{2}$. Indeed, the following nontrivial elements $x \in S^{1}$ have conjugate images (in $\mathrm{U}(7)$, hence in $G_{2}$ ) under the two representations.

$$
\begin{gathered}
\left(W_{1}, W_{3}\right): x=-1 \\
\left(W_{1}, W_{4}\right): x=\zeta_{3} \\
\left(W_{1}, W_{28}\right): x=\zeta_{3} \\
\left(W_{3}, W_{28}\right): x=\zeta_{5} \\
\left(W_{4}, W_{28}\right): x=\zeta_{3}
\end{gathered}
$$

If $\mathrm{SU}(2)$ acts on $G_{2}$ by $\left(W_{3}, W_{4}\right)$, however, then we compute from the above formulas that the two images of any nontrivial element $x \in$ $S^{1} \subset \mathrm{SU}(2)$ are not conjugate in $\mathrm{U}(7)$, and hence not conjugate in $G_{2}$. So this is a free action of $\mathrm{SU}(2)$ on $G_{2}$. Since the Dynkin indices 3 and 4 differ by 1 , the resulting biquotient $M=G_{2} / \mathrm{SU}(2)$ has $\pi_{3} M=0$. Using the known homology of $G_{2}$, we compute that the 11-manifold $M$ is 4-connected and has the integral homology groups of $\mathrm{UT}\left(S^{6}\right)$.

Thus, the only biquotient $G_{2} / H$ with $H$ isomorphic to $\mathrm{SU}(2)$ or $\mathrm{SO}(3)$ and $H$ acting nontrivially on both sides is the 4-connected 11manifold $G_{2} / \mathrm{SU}(2)$, with $\mathrm{SU}(2)$ acting by $\left(W_{3}, W_{4}\right)$.

\section{Three Cheeger manifolds which are not homotopy equivalent to biquotients}

We now return to the proof of Theorem 2.1. Roughly in order of increasing difficulty, we show in this section that the 16-manifolds $\mathbf{C a P}^{2} \# \mathbf{C a P}{ }^{2}, \mathbf{C P}^{8} \# \mathbf{C a P} \mathbf{P}^{2}$, and $\mathbf{H P}^{4} \# \mathbf{C a P}^{2}$ are not homotopy equivalent to biquotients.

Suppose that the 16-manifold $\mathbf{C} \mathbf{a} \mathbf{P}^{2} \# \mathbf{C a} \mathbf{P}^{2}$ is homotopy equivalent to a biquotient $M=G / H$. As throughout the paper, we assume that 
$M$ is written as a biquotient $G / H$ which satisfies Convention 3.4. Here $H^{*}(M, \mathbf{Z}) \cong \mathbf{Z}[x, y] /\left(x y=0, x^{2}=y^{2}\right),|x|=|y|=8$. This is a complete intersection ring with 2 generators in degree 8 and 2 relations in degree 16. It follows that the rational homotopy groups of $M$ are isomorphic to $\mathrm{Q}^{2}$ in dimensions 8 and 15 , and otherwise 0 (the same as for $S^{8} \times S^{8}$ ). So the map $\pi_{2 d-1} H_{\mathbf{Q}} \rightarrow \pi_{2 d-1} G_{\mathbf{Q}}$ must have 2-dimensional cokernel for $d=8,2$-dimensional kernel for $d=4$, and otherwise is an isomorphism. Equivalently, we say that $G$ contributes two degrees 8 to $M$, while $H$ contributes two degrees 4 , and nothing else.

Each simple factor of $G$ contributes at least one degree to $M$, by Theorem 4.8, so $G$ has at most two simple factors. Suppose first that $G$ is simple. We know that $G$ contributes two degrees 8 to $M$, and nothing else. One of the four cases in Theorem 4.8 must hold. Here (1) cannot hold: if $G$ contributes its maximal degree to $M$, that would have to be 8 , but the maximal degree of each simple Lie group occurs only with multiplicity 1 . The remaining cases of the theorem are incompatible with the fact that $G$ contributes only degree 8 to $M$. Thus we have a contradiction from the assumption that $G$ is simple.

So $G$ has two simple factors, $G=G_{1} \times G_{2}$. (Here $G_{2}$ does not denote the exceptional group $G_{2}$.) Each factor must contribute one degree 8 to $M$, and nothing else. We can apply Theorem 4.8 to each factor $G_{i}$. From the degrees, it is clear that only cases (1) and (3) can occur. That is, for $1 \leq i \leq 2$, either $G_{i}$ contributes its maximal degree to $M$, which must be 8 , or else $G_{i}=\operatorname{Spin}(16)$ and there is a simple factor $H_{i} \cong \operatorname{Spin}(15)$ of $H$ which acts nontrivially on exactly one side of $G_{i}$, with $G_{i} / H_{i}=S^{15}$. If $G_{i}$ contributes its maximal degree to $M$, we can apply Theorem 5.3 to $G_{i}$. Cases (1) and (2) cannot arise, since the maximal degree of $G_{i}$ is 8 . Therefore, by cases (3) and (4), there is a simple factor $H_{i}$ of $H$ which acts nontrivially on exactly one side of $G_{i}$ such that $G_{i} / H_{i}$ is one of the homogeneous spaces $\operatorname{Spin}(9) / \operatorname{Spin}(8)=S^{8}, \mathrm{SU}(8) / \mathrm{SU}(7)=$ $\operatorname{Sp}(8) / \operatorname{Sp}(6)=\operatorname{Spin}(9) / \operatorname{Spin}(7)=S^{15}, \operatorname{Spin}(9) / \operatorname{Spin}(7)=\mathrm{UT}\left(S^{8}\right)$, $\operatorname{Spin}(10) / \operatorname{Spin}(8), \operatorname{Spin}(10) / \operatorname{Spin}(7), \operatorname{Spin}(9) / G_{2}$, or $\operatorname{Spin}(10) / G_{2}$.

Since neither $G$ nor $H$ contributes any degree 2 to $M$, and each simple group has exactly one degree 2 (that is, every simple group has $\left.\pi_{3}=\mathbf{Z}\right), H$ must have the same number of simple factors as $G$. Thus $H$ has two simple factors.

Let $G_{1}$ and $G_{2}$ denote the two simple factors of $G$. We know that there is a simple factor $H_{1}$ of $H$ such that $H_{1}$ acts nontrivially on exactly one side of $G_{1}$, with $G_{1} / H_{1}$ equal to either $\operatorname{Spin}(16) / \operatorname{Spin}(15)=S^{15}$ or one of the other homogeneous spaces listed above. We also know the 
analogous statement for $G_{2}$, but a priori it is possible that the same simple factor $H_{1}$ of $H$ plays the same role for both $G_{1}$ and $G_{2}$. But then $G=G_{1} \times G_{2}$ has two degrees 14 (if $G_{1}$ and $G_{2}$ are Spin(16)) or 7 (if $G_{1}$ and $G_{2}$ are $\mathrm{SU}(8)$ ) or 6 (if $G_{1}$ and $G_{2}$ are $\operatorname{Spin}(9)$ or $\operatorname{Spin}(10)$ ), both of which must be killed by $H$, whereas the group $H_{1}$ has only one degree 14 or 6 or 7 . So we can order the two simple factors of $H$ in such a way that $H_{i}$ kills the relevant degree of $G_{i}$, both for $i=1$ and for $i=2$. By the proof of Theorem 5.3, it follows that $H_{i}$ acts nontrivially on exactly one side of $G_{i}$, with $G_{i} / H_{i}$ equal to one of the above homogeneous spaces, for $i=1$ and for $i=2$.

Since $M$ has dimension only 16 , both $G_{1} / H_{1}$ and $G_{2} / H_{2}$ must be the homogeneous space $\operatorname{Spin}(9) / \operatorname{Spin}(8)=S^{8}$. If $H_{1}$ acts trivially on $G_{2}$, or also if $H_{2}$ acts trivially on $G_{1}$, then $M$ is an $S^{8}$-bundle over $S^{8}$ and hence has signature zero, contradicting the fact that $M$ is homotopy equivalent to $\mathbf{C a P}^{2} \# \mathbf{C a P}{ }^{2}$. So $H_{1}$ acts nontrivially on $G_{2}$ and $H_{2}$ acts nontrivially on $G_{1}$. Since $H_{i}$ has finite centralizer in $G_{i}$ for $i=1,2$, the action of $H_{1}$ on $G_{2}$ must be given by a nontrivial homomorphism $H_{1} \rightarrow G_{2}$ on the other side of $G_{2}$ from $H_{2}$, and likewise for the action of $H_{2}$ on $G_{1}$. Any nontrivial homomorphism $\operatorname{Spin}(8) \rightarrow \operatorname{Spin}(9)$ has Dynkin index 1 . So the homomorphism $\pi_{3} H \rightarrow \pi_{3} G, \mathbf{Z}^{2} \rightarrow \mathbf{Z}^{2}$, is given by a $2 \times 2$ matrix with both rows equal to $(1,-1)$ or $(-1,1)$. Such a matrix has zero determinant, and so $\pi_{3} M_{\mathbf{Q}}$ is not zero. Again, this contradicts the fact that $M$ is homotopy equivalent to $\mathbf{C a P}^{2} \# \mathbf{C a} \mathbf{P}^{2}$. This completes the proof that $\mathbf{C a P}^{2} \# \mathbf{C a P} \mathbf{P}^{2}$ is not homotopy equivalent to a biquotient. In fact, the proof shows that $\mathbf{C a P}^{2} \# \mathbf{C a P}{ }^{2}$ is not even rationally homotopy equivalent to a biquotient.

We now prove that $\mathbf{C P}^{8} \# \mathbf{C a} \mathbf{P}^{2}$ is not homotopy equivalent to a biquotient $M=G / K$. Since $G$ is simply connected, the boundary map $\mathbf{Z} \cong \pi_{2} M \rightarrow \pi_{1} K$ in the long exact sequence is an isomorphism.

So, if we let $H$ be the commutator subgroup of $K$, then $H$ is simply connected and $K / H$ is isomorphic to $S^{1}$. Let $N$ be the 17 -manifold $G / H$. Since $N$ is the natural $S^{1}$-bundle over $M \simeq \mathbf{C P}^{8} \# \mathbf{C a P}^{2}$, we compute that $N$ has the integral cohomology ring of $S^{8} \times S^{9}$. To go further, we use Wu's theorem that the Stiefel-Whitney classes of the tangent bundle of $M$ are invariants of the homotopy type of $M$ ([19], Theorem 11.14). Because the 8-sphere in the Cayley plane has selfintersection 1, the Stiefel-Whitney class $w_{8}\left(\mathbf{C a P}^{2}\right) \in H^{8}\left(\mathbf{C a P}^{2}, \mathbf{F}_{2}\right)$ is not zero. Therefore $w_{8}\left(\mathbf{C P}^{8} \# \mathbf{C a} \mathbf{P}^{2}\right)$ is not in the subgroup $\left(H^{2}\right)^{4}$ of $H^{8}$. By Wu's theorem, the same holds for the manifold $M$ which is homotopy equivalent to $\mathbf{C P}^{8} \# \mathbf{C a} \mathbf{P}^{2}$. Since $N$ is an $S^{1}$-bundle over $M$, 
it follows that $w_{8}(N)$ is not zero. We will use this later.

The cohomology ring of $M$ is a complete intersection ring. The degrees of the generators and relations determine the rational homotopy groups of $M$, and hence of $N$. The result is that $G$ contributes degrees 5 and 8 to $N$, while $H$ contributes 4 , and nothing else. Since every simple factor of $G$ contributes at least one degree to $N, G$ has at most 2 simple factors. Also, since neither $G$ nor $H$ contributes degree 2, $G$ and $H$ have the same number of simple factors.

Suppose first that $G$ is simple. It follows that $H$ is simple, too. We can apply Theorem 4.8, and cases (2), (3), (4) are excluded since $G$ contributes degrees 5 and 8 to $N$ and no more. Therefore case (1) must hold; that is, $G$ contributes its maximal degree to $N$. So $G$ has maximal degree 8 . There is no simple group whose highest two degrees are 5 and 8, so the second-largest degree of $G$ must be killed by $H$. Since $G$ has exactly one degree $8, H$ must have maximal degree less than 8. So, regardless of how $H$ acts on $G,(G, H)$ must be one of the pairs in Lemma 5.2. From the list there, since we know that $G$ adds degrees 5 and 8 to $M$ and $H$ adds degree 4 and no more, we must have $G=\operatorname{Spin}(10)$ and $H=\operatorname{Spin}(8)$. All nontrivial homomorphisms $H \rightarrow G$ have Dynkin index 1. So if $H$ acts nontrivially on both sides of $G$, then $G$ would contribute degree 2 to $M$, a contradiction. So $H$ acts on only one side of $G$ and $G / H$ is isomorphic to the homogeneous space $\operatorname{Spin}(10) / \operatorname{Spin}(8)=\mathrm{UT}\left(S^{9}\right)$.

Indeed, there is a free $S^{1}$-action on $\mathrm{UT}\left(S^{9}\right)$, with quotient the 8dimensional complex quadric $Q_{\mathrm{C}}^{8}$. This quadric has the rational homotopy type of $\mathbf{C P}^{8} \# \mathbf{C a P}{ }^{2}$. But no $S^{1}$-quotient of $N=\mathrm{UT}\left(S^{9}\right)$ can have the homotopy type of $\mathbf{C P}^{8} \# \mathbf{C} \mathbf{a} \mathbf{P}^{2}$. The point is that $N$ is an $S^{8}$-bundle over $S^{9}$. It follows that there is a sphere $S^{8}$ in $N$ which represents a generator of $H_{8}\left(N, \mathbf{F}_{2}\right)$ and which has trivial normal bundle. Therefore $w_{8}(N) \mid S^{8}=0$, and hence $w_{8}(N)=0$, contradicting our earlier calculation that the Stiefel-Whitney class $w_{8}(N)$ is not zero.

So $G$ must have two simple factors. Since $G$ and $H$ have the same number of simple factors, $H$ also has two simple factors. Each simple factor of $G$ must contribute exactly one degree to $N$; we can assume that $G_{1}$ contributes degree 8 and $G_{2}$ contributes degree 5 . By Theorems 4.8 and 5.3, there is a simple factor $H_{1}$ acting on exactly one side of $G_{1}$ such that $G_{1} / H_{1}$ is one of the homogeneous spaces $\operatorname{Spin}(9) / \operatorname{Spin}(8)=S^{8}$, $\operatorname{Spin}(16) / \operatorname{Spin}(15)=\mathrm{SU}(8) / \mathrm{SU}(7)=\operatorname{Sp}(8) / \mathrm{Sp}(6)=\operatorname{Spin}(9) / \operatorname{Spin}(7)$ $=S^{15}, \operatorname{Spin}(9) / \operatorname{Spin}(7)=\mathrm{UT}\left(S^{8}\right), \operatorname{Spin}(10) / \operatorname{Spin}(8), \operatorname{Spin}(10) / \operatorname{Spin}(7)$, $\operatorname{Spin}(9) / G_{2}$, or $\operatorname{Spin}(10) / G_{2}$. Also, by the same theorems, there is a 
simple factor $H_{2}$ acting on exactly one side of $G_{2}$ such that $G_{2} / H_{2}$ is one of the homogeneous spaces $\operatorname{Spin}(10) / \operatorname{Spin}(9)=\mathrm{SU}(5) / \mathrm{SU}(4)=S^{9}$ or $\mathrm{SU}(6) / \mathrm{Sp}(6)$.

If $H_{1}$ and $H_{2}$ are the same factor of $H$, this factor must be isomorphic to $\operatorname{Sp}(6)$, and $N$ is a biquotient of the form $(\operatorname{Sp}(8) \times \mathrm{SU}(6)) /(\operatorname{Sp}(6) \times X)$. Here $X$ must be a simple group with degrees $2,3,4,4,6$, but there is no such group. So $H_{1}$ and $H_{2}$ are the two different simple factors of $H$.

From the degrees of $N$ (or just by its dimension, which is only 17 ), the homogeneous space $G_{1} / H_{1}$ must be $\operatorname{Spin}(9) / \operatorname{Spin}(8)=S^{8}$ and the homogeneous space $G_{2} / H_{2}$ must be either $\operatorname{Spin}(10) / \operatorname{Spin}(9)=S^{9}$ or $\mathrm{SU}(5) / \mathrm{SU}(4)=S^{9}$. The 17 -manifold $N$ is not an $S^{8}$-bundle over $S^{9}$, because we know that $w_{8}(N)$ is not zero. So $H_{1}=\operatorname{Spin}(8)$ must act nontrivially on the second factor $G_{2}$ of $G$. It follows that $G_{2} / H_{2}$ is $\operatorname{Spin}(10) / \operatorname{Spin}(9)=S^{9}$, not $\mathrm{SU}(5) / \mathrm{SU}(4)=S^{9}$. Furthermore, $H_{2}=$ $\operatorname{Spin}(9)$ must act trivially on $G_{1}=\operatorname{Spin}(9)$, by Lemma 4.1. Thus $N$ is a biquotient of the form $(\operatorname{Spin}(9) \times \operatorname{Spin}(10)) /(\operatorname{Spin}(8) \times \operatorname{Spin}(9))$ with Spin(9) acting trivially on $\operatorname{Spin}(9)$ and $\operatorname{Spin}(8)$ acting nontrivially on $\operatorname{Spin}(10)$. That is, $N$ is a nontrivial $S^{9}$-bundle over $S^{8}$.

There are three conjugacy classes of nontrivial homomorphisms from $\operatorname{Spin}(8)$ to $\operatorname{Spin}(10)$. They have the form $W \oplus \mathbf{R}^{2}$, where $W$ is either the standard 8-dimensional real representation $V$ of $\operatorname{Spin}(8)$ or one of the two spin representations $S^{-}$and $S^{+}$. Thus $N$ is the $S^{9}$ bundle $S\left(W \oplus \mathbf{R}^{2}\right)$ over $S^{8}$, where $W$ is the real vector bundle over $\operatorname{Spin}(9) / \operatorname{Spin}(8)=S^{8}$ corresponding to the representation $W$ of $\operatorname{Spin}(8)$. If $W$ is the standard representation $V$ of $\operatorname{Spin}(8)$, then the corresponding vector bundle on $S^{8}$ is the tangent bundle, which has zero StiefelWhitney classes. It follows that the sphere bundle $S\left(V \oplus \mathbf{R}^{2}\right)$ has zero Stiefel-Whitney classes, contrary to what we know about $N$. So $W$ must be one of the two spin representations of $\operatorname{Spin}(8)$. Without loss of generality, we can assume that $W=S^{-}$; if instead $W=S^{+}$, we can apply an automorphism of order 2 of $\operatorname{Spin}(8)$ which switches the isomorphism classes of the representations $S^{-}$and $S^{+}$and does not change the isomorphism class of the representation $V$ and hence of the standard inclusion $\operatorname{Spin}(8) \rightarrow \operatorname{Spin}(9)$. Once that is done, $N$ is the $S^{9}$-bundle $S\left(S^{-} \oplus \mathbf{R}^{2}\right)$ over $S^{8}$.

Finally, we need to analyze the free $S^{1}$-action on $N$ that gives $M=$ $N / S^{1}$. By computing the centralizers of the homomorphisms that define $N$, we see that the $S^{1}$-action on $N$ is defined by a homomorphism $S^{1} \rightarrow$ Spin(9) together with a homomorphism from $S^{1}$ to $S^{1}$, the identity component of the centralizer of $\operatorname{Spin}(8)$ in $\operatorname{Spin}(10)$. The homomorphism 
$S^{1} \rightarrow \operatorname{Spin}(9)$ defines an action of $S^{1}$ on $S^{8}$ and also on the $\operatorname{Spin}(9)-$ equivariant vector bundle $S^{-}$over $S^{9}$, while the homomorphism $S^{1} \rightarrow$ $S^{1}$, of the form $z \mapsto z^{b}$ for some integer $b$, defines the action of $S^{1}$ on the trivial bundle $\mathbf{R}^{2}$ over $S^{8}$. Since $S^{1}$ is acting freely on $N=$ $S\left(S^{-} \oplus \mathbf{R}^{2}\right)$, it must act freely on both $S\left(S^{-}\right)=S^{15}$ and on $S\left(\mathbf{R}^{2}\right)=$ $S^{1} \times S^{8}$. Since the $S^{1}$-action on $S^{8}$ must have a fixed point, the freeness of the $S^{1}$-action on $S\left(\mathbf{R}^{2}\right)$ means that $b= \pm 1$. We compute that there is a unique conjugacy class of homomorphisms $S^{1} \rightarrow \operatorname{Spin}(9)$ which give a free action of $S^{1}$ on $S\left(S^{-}\right)=S^{15}$, namely the subgroup $S^{1}=$ $\operatorname{Spin}(2) \subset \operatorname{Spin}(9)$. We can assume that $b=1$, since changing $b=-1$ to $b=1$ clearly does not change the diffeomorphism class of the quotient manifold $M=N / S^{1}$. Thus we have uniquely described the biquotient $M$.

But the biquotient $M$ we have described is exactly the one which we proved to be diffeomorphic to $\mathbf{C P}^{8} \#-\mathbf{C a P}^{2}$ in Section 2. In particular, it is not homotopy equivalent to $\mathbf{C P}^{8} \# \mathbf{C a} \mathbf{P}^{2}$. This completes the proof that $\mathbf{C P}^{8} \# \mathbf{C a P}^{2}$ is not homotopy equivalent to a biquotient.

We now show that $\mathbf{H P}^{4} \# \mathbf{C a} \mathbf{P}^{2}$ is not homotopy equivalent to a biquotient $M=G / H$. Since $M$ is 2-connected, $H$ is simply connected, by Lemma 3.1. The ring $H^{*}(M, \mathbf{Z})$ is a complete intersection, and so the degrees of its generators and relations determine the rational homotopy groups of $M$. The result is that $G$ contributes degrees 6 and 8 to $M$, while $H$ contributes degrees 2 and 4 , and no others. Since every simple factor of $G$ contributes at least one degree to $M, G$ has at most 2 simple factors. Since $H$ contributes exactly one degree $2, H$ has one more simple factor than $G$ has.

Suppose first that $G$ is simple. We can apply Theorem 4.8 , and cases (2), (3), (4) are excluded because $G$ contributes degrees 6 and 8 to $M$ and no more. Therefore case (1) must hold; that is, $G$ contributes its maximal degree to $M$. So $G$ has maximal degree 8. By Table 4.2, $G$ must be one of $\mathrm{Sp}(8)$, $\operatorname{Spin}(9)$, $\mathrm{Spin}(10)$, or $\mathrm{SU}(8)$, which have degrees respectively $2,4,6,8,2,4,6,8,2,4,5,6,8$, or $2,3,4,5,6,7,8$. It follows that $H$ has, correspondingly, degrees $2,2,4,4,2,2,4,4,2,2,4,4,5$, or $2,2,3,4,4,5,7$. But there is no semisimple group $H$ with the last two sets of degrees: one simple factor of $H$ would have to have maximal degree equal to an odd number $d$ ( 5 or 7 ), hence would be isomorphic to $\mathrm{SU}(d)$, and hence would have all degrees from 2 to $d$, which is not the case here. Therefore $G$ is isomorphic to $\operatorname{Sp}(8)$ or $\operatorname{Spin}(9)$, and $H$ has degrees $2,2,4,4$. Since $H$ is simply connected, Table 4.2 shows that $H$ is isomorphic to $\operatorname{Sp}(4)^{2}$. 
We compute that there is no free action of $\operatorname{Sp}(4)^{2}$ on $\operatorname{Spin}(9)$, and that the only free action of $\operatorname{Sp}(4)^{2}$ on $\operatorname{Sp}(8)$ is the one-sided action given by the standard inclusion $\operatorname{Sp}(4)^{2} \subset \operatorname{Sp}(8)$. So $M$ is the homogeneous space $\operatorname{Sp}(8) / \operatorname{Sp}(4)^{2}$. This homogeneous space has the rational homotopy type of $\mathbf{H P}^{4} \# \mathbf{C a P}^{2}$, but not the homotopy type, because we compute that $w_{4}\left(\operatorname{Sp}(8) / \operatorname{Sp}(4)^{2}\right)=0$ whereas $w_{4}\left(\mathbf{H P}^{4} \# \mathbf{C a P} \mathbf{P}^{2}\right) \neq 0$, since $w_{4}\left(\mathbf{H P}^{4}\right) \neq 0$. To compute these Stiefel-Whitney classes of the homogeneous spaces $\operatorname{Sp}(8) / \operatorname{Sp}(4)^{2}$ and $\mathbf{H P}^{4}$, we can use Singhof's approach, which works more generally for biquotients [25]. Namely, say for $X=\operatorname{Sp}(8) / \operatorname{Sp}(4)^{2}$, the tangent bundle is

$$
T X=s p(8)-s p(4)_{1}-s p(4)_{2}
$$

in the Grothendieck group of real vector bundles on $X$. The groups $H^{i}\left(\mathrm{BSp}(2 n), \mathbf{F}_{2}\right)$ are zero for $0<i<4$, and so

$$
w_{4} X=w_{4}(s p(8))-w_{4}\left(s p(4)_{1}\right)-w_{4}\left(s p(4)_{2}\right) .
$$

This is zero because $w_{4}\left(s p(4)_{1}\right)+w_{4}\left(s p(4)_{2}\right)$ is clearly some $\mathbf{F}_{2}$-multiple of the sum of the generators of $H^{4}\left(\mathrm{BSp}(4)_{1}, \mathbf{F}_{2}\right)$ and of $H^{4}\left(\mathrm{BSp}(4)_{2}, \mathbf{F}_{2}\right)$, while the generator of $H^{4}\left(\operatorname{BSp}(8), \mathbf{F}_{2}\right)$ pulls back to the sum of the generators of $H^{4}\left(\operatorname{BSp}(4)_{1}, \mathbf{F}_{2}\right)$ and $H^{4}\left(\operatorname{BSp}(4)_{2}, \mathbf{F}_{2}\right)$ and also pulls back to zero in $H^{4}\left(X, \mathbf{F}_{2}\right)$.

Therefore $G$ is not simple. It must have two simple factors, each of which contributes exactly one degree to $M$. We know that $H$ has one more simple factor than $G$, so $H$ has three simple factors. We can write $G=G_{1} \times G_{2}$ where $G_{1}$ contributes degree 6 to $M$ and $G_{2}$ contributes degree 8 to $M$, and nothing else. By Theorems 4.8 and 5.3 applied to $G_{1}$, there is a simple factor $H_{1}$ of $H$ such that either $G_{1}$ is the exceptional group $G_{2}, H_{1}$ is $\mathrm{SU}(2)$, and $H_{1}$ acts nontrivially on $G_{1}$, or $H_{1}$ acts nontrivially on exactly one side of $G_{1}$ and $G_{1} / H_{1}$ is isomorphic to one of the homogeneous spaces $\operatorname{Spin}(7) / \operatorname{Spin}(6)=$ $G_{2} / \mathrm{SU}(3)=S^{6}, \operatorname{Spin}(12) / \operatorname{Spin}(11)=\mathrm{SU}(6) / \mathrm{SU}(5)=\mathrm{Sp}(6) / \mathrm{Sp}(4)=$ $S^{11}, \operatorname{Spin}(7) / \operatorname{Spin}(5)=\mathrm{UT}\left(S^{6}\right), \operatorname{Spin}(8) / \operatorname{Spin}(6)$, or $\operatorname{Spin}(8) / \operatorname{Spin}(5)$. Likewise, by Theorems 4.8 and 5.3 applied to the second factor $G_{2}$, there is a simple factor $H_{2}$ of $H$ which acts nontrivially on exactly one side of $G_{2}$ with $G_{2} / H_{2}$ isomorphic to one of the homogeneous spaces $\operatorname{Spin}(9) / \operatorname{Spin}(8)=S^{8}, \operatorname{Spin}(16) / \operatorname{Spin}(15)=\mathrm{SU}(8) / \mathrm{SU}(7)=$ $\operatorname{Sp}(8) / \operatorname{Sp}(6)=\operatorname{Spin}(9) / \operatorname{Spin}(7)=S^{15}, \operatorname{Spin}(9) / \operatorname{Spin}(7)=\mathrm{UT}\left(S^{8}\right)$, $\operatorname{Spin}(10) / \operatorname{Spin}(8), \operatorname{Spin}(10) / \operatorname{Spin}(7), \operatorname{Spin}(9) / G_{2}$, or $\operatorname{Spin}(10) / G_{2}$.

We see that in all the above cases $G_{1} / H_{1}$ and $G_{2} / H_{2}, H_{1}$ is never isomorphic to $H_{2}$, so in particular $H_{1}$ is not the same simple factor of $H$ 
as $H_{2}$. So, given $G_{1} / H_{1}$ and $G_{2} / H_{2}$, the known degrees of $M$ determine the degrees of the remaining simple factor of $H, H_{3}$. Let us say that a given set of factors of $G$ and $H$, for example $G_{1}$ and $H_{1}$, adds a degrees $d$ to $M$ if the integer $a$ is the number of degrees $d$ in the given factors of $G$ minus the number of degrees $d$ in the given factors of $H$. From the above list, $G_{1} / H_{1}$ always adds exactly one degree 6 to $M$, while $G_{2} / H_{2}$ adds none; so, by the known degrees of $M, H_{3}$ has no degree 6 . The only simple group with two degrees 4 is $\operatorname{Spin}(8)$, which also has a degree 6 ; so $H_{3}$ has at most one degree 4.

Also, from the above list, $G_{2} / H_{2}$ adds no degree 3 to $M$, so the cases where $G_{1} / H_{1}$ adds -1 degree 3 to $M$ cannot occur (otherwise $H_{3}$ would have -1 degrees 3 ). Thus $G_{1} / H_{1}$ is not $\operatorname{Spin}(7) / \operatorname{Spin}(6)=S^{6}$, $G_{2} / \mathrm{SU}(3)=S^{6}$, or $\operatorname{Spin}(8) / \operatorname{Spin}(6)=\mathrm{UT}\left(S^{7}\right)$. Thus $G_{1} / H_{1}$ and $G_{2} / H_{2}$ both add zero degrees 3 to $M$, and so $H_{3}$ has no degree 3 . Next, we observe that $G_{1} / H_{1}$ adds $\geq 0$ degrees 4 to $M$, so the cases where $G_{2} / H_{2}$ adds one degree 4 to $M$ cannot occur (otherwise $H_{3}$ would have at least two degrees 4). Thus $G_{2} / H_{2}$ is not $\operatorname{Spin}(9) / G_{2}$ or $\operatorname{Spin}(10) / G_{2}$. Next, $G_{1} / H_{1}$ adds zero degrees 5 to $M$, so in the cases where $G_{2} / H_{2}$ adds degree 5 to $M, H_{3}$ must have a degree 5 . Since $H_{3}$ has no degree 6 , it follows that $H_{3}$ is isomorphic to $\mathrm{SU}(5)$, contradicting the fact that $H_{3}$ has no degree 3. Thus $G_{2} / H_{2}$ is not $\operatorname{Spin}(10) / \operatorname{Spin}(8)$ or $\operatorname{Spin}(10) / \operatorname{Spin}(7)$.

Finally, if $G_{1} / H_{1}$ is $\operatorname{Spin}(8) / \operatorname{Spin}(5)=\operatorname{Spin}(8) / \operatorname{Sp}(4)$, then $G_{1} / H_{1}$ adds one degree 4 to $M$, and so, since $H_{3}$ has at most one degree 4 , $G_{2} / H_{2}$ must add -1 degrees 4 to $M$; that is, $G_{2} / H_{2}$ is $\operatorname{Spin}(9) / \operatorname{Spin}(8)=$ $S^{8}$. Then $H_{3}$ has degrees 2,4 and so $H_{3}$ is isomorphic to $\operatorname{Sp}(4)$. Here $M$ is a biquotient $(\operatorname{Spin}(8) \times \operatorname{Spin}(9)) /(\operatorname{Sp}(4) \times \operatorname{Spin}(8) \times \operatorname{Sp}(4))$. Since $H_{2}=\operatorname{Spin}(8)$ has finite centralizer in $G_{2}=\operatorname{Spin}(9)$, the two $\operatorname{Sp}(4)$ factors of $H$ can only act on $\operatorname{Spin}(9)$ on the other side from $\operatorname{Spin}(8)$; so $\operatorname{Sp}(4)^{2}$ acts on $S^{8}$. Looking at the low-dimensional orthogonal representations of $\operatorname{Sp}(4)^{2}$ shows that this action must have a fixed point. Since $H$ acts freely on $G$, it follows that there is a subgroup of $H$ isomorphic to $\operatorname{Sp}(4)^{2}$ which acts freely on $\operatorname{Spin}(8)$. We compute, however, that there is no free action of $\operatorname{Sp}(4)^{2}$ on $\operatorname{Spin}(8)$. This contradiction shows that $G_{1} / H_{1}$ is not $\operatorname{Spin}(8) / \operatorname{Spin}(5)=\operatorname{Spin}(8) / \operatorname{Sp}(4)$.

The only remaining possibilities for $G_{1} / H_{1}$ are those which add degree 6 to $M$ and nothing else: a homogeneous space $\operatorname{Spin}(12) / \operatorname{Spin}(11)=$ $\mathrm{SU}(6) / \mathrm{SU}(5)=\operatorname{Sp}(6) / \mathrm{Sp}(4)=S^{11}$ or $\operatorname{Spin}(7) / \operatorname{Spin}(5)=\mathrm{UT}\left(S^{6}\right)$ or $\left(G_{1}, H_{1}\right)=\left(G_{2}, A_{1}\right)$ (the exceptional group $\left.G_{2}\right)$. Also, $G_{2} / H_{2}$ is either $\operatorname{Spin}(9) / \operatorname{Spin}(8)=S^{8}$, which adds one degree 8 and subtracts one de- 
gree 4 from $M$, or else a homogeneous space which adds degree 8 to $M$ and nothing else: $\operatorname{Spin}(16) / \operatorname{Spin}(15)=\mathrm{SU}(8) / \mathrm{SU}(7)=\mathrm{Sp}(8) / \mathrm{Sp}(6)=$ $\operatorname{Spin}(9) / \operatorname{Spin}(7)=S^{15}$ or $\operatorname{Spin}(9) / \operatorname{Spin}(7)=\mathrm{UT}\left(S^{8}\right)$. From the known degrees of $M$, it follows that $H_{3}$ has only degree 2 if $G_{2} / H_{2}$ is $S^{8}$ and has degrees 2, 4 otherwise. Therefore $H_{3}$ is isomorphic to $\mathrm{SU}(2)$ if $G_{2} / H_{2}$ is $S^{8}$ and to $\operatorname{Sp}(4)$ otherwise.

Suppose that $H_{3}$ is isomorphic to $\mathrm{Sp}(4)$; we will derive a contradiction. First, we can easily exclude the possibility that the first factor $G_{1}$ is the exceptional group $G_{2}$. The point is that, in this case, neither $H_{3}=\mathrm{Sp}(4)$ nor $H_{2}$ (from the list, above) has a nontrivial homomorphism to the first factor $G_{1}$. Since $H_{2} \times H_{3} \subset H$ must act freely on $G_{1} \times G_{2}$, it follows that $H_{2} \times H_{3}$ acts freely on the second factor $G_{2}$. This is impossible because the list of possible spaces $G_{2} / H_{2}$ shows that $H_{2}$ has rank 1 less than the second factor $G_{2}$, and so $H_{2} \times H_{3}=H_{2} \times \operatorname{Sp}(4)$ has rank 1 greater than the second factor $G_{2}$. The impossibility here follows from the fact that a finite-dimensional elliptic space $X$ has the alternating sum of its rational homotopy groups $\chi_{\pi}(X) \leq 0$, by Halperin ([13], p. 434). So the first factor $G_{1}$ is not the exceptional group $G_{2}$.

We continue to assume that $H_{3}$ is $\operatorname{Sp}(4)$. We know that $G_{1} / H_{1}$ is a homogeneous space $\operatorname{Spin}(12) / \operatorname{Spin}(11)=\mathrm{SU}(6) / \mathrm{SU}(5)=\mathrm{Sp}(6) / \mathrm{Sp}(4)$ $=S^{11}$ or $\operatorname{Spin}(7) / \operatorname{Spin}(5)=\mathrm{UT}\left(S^{6}\right)$, and $G_{2} / H_{2}$ is a homogeneous space $\operatorname{Spin}(16) / \operatorname{Spin}(15)=\mathrm{SU}(8) / \mathrm{SU}(7)=\mathrm{Sp}(8) / \mathrm{Sp}(6)=$ $\operatorname{Spin}(9) / \operatorname{Spin}(7)=S^{15}$ or $\operatorname{Spin}(9) / \operatorname{Spin}(7)=\mathrm{UT}\left(S^{8}\right)$. For each possible $G_{1} / H_{1}$ and $G_{2} / H_{2}$, we check immediately that either $H_{1}$ must act trivially on $G_{2}$ or $H_{2}$ must act trivially on $G_{1}$. Here we use in particular that a simple factor of $H$ must act trivially on a simple factor of $G$ isomorphic to it, by Lemma 4.1. It follows that the biquotient $\left(G_{1} \times G_{2}\right) /\left(H_{1} \times H_{2}\right)$ is either a $G_{1} / H_{1}$-bundle over $G_{2} / H_{2}$ or a $G_{2} / H_{2}$ bundle over $G_{1} / H_{1}$. Now $G_{1} / H_{1}$ is either $S^{11}$ or $\mathrm{UT}\left(S^{6}\right)$, so it has the 3-local homotopy type of $S^{11}$; and likewise $G_{2} / H_{2}$ is either $S^{15}$ or $\mathrm{UT}\left(S^{8}\right)$, so it has the 3-local homotopy type of $S^{15}$. Therefore the biquotient $\left(G_{1} \times G_{2}\right) /\left(H_{1} \times H_{2}\right)$ is 3-locally 11-connected. It follows that the natural map

$$
M=\left(G_{1} \times G_{2}\right) /\left(H_{1} \times H_{2} \times \mathrm{Sp}(4)\right) \rightarrow \operatorname{BSp}(4)
$$

is 3-locally 11-connected. Up to this point, any odd prime number would serve in place of 3 , but we now derive a contradiction by a 3 local calculation. Since $M$ is homotopy equivalent to $\mathbf{H} \mathbf{P}^{4} \# \mathbf{C a P} \mathbf{P}^{2}$, the map $\pi_{8} M \otimes H^{8}(M, \mathbf{Z}) \rightarrow \mathbf{Z}$ is surjective. On the other hand, the map $\pi_{8} \mathrm{BSp}(4) \times H^{8}(\operatorname{BSp}(4), \mathbf{Z}) \rightarrow \mathbf{Z}$ has image $6 \mathbf{Z}$. Indeed, $H^{8}(\operatorname{BSp}(4), \mathbf{Z})$ 
is generated by $c_{2}^{2}$ and $c_{4}$, and $c_{2}^{2}$ is clearly zero for any $\mathrm{Sp}(4)$-bundle over $S^{8}$, while $c_{4}$ of such a bundle is a multiple of $(4-1) !=6$ by Bott periodicity. Since the above map $M \rightarrow \mathrm{BSp}(4)$ is 3-locally 11-connected, we have a contradiction.

That shows that $H_{3}$ is not isomorphic to $\operatorname{Sp}(4)$. Therefore $H_{3}$ is isomorphic to $\mathrm{SU}(2)$. In this case, we know that $G_{2} / H_{2}$ is $\operatorname{Spin}(9) / \operatorname{Spin}(8)$ $=S^{8}$. Also, $G_{1} / H_{1}$ is either a homogeneous space $\operatorname{Spin}(12) / \operatorname{Spin}(11)$ $=\mathrm{SU}(6) / \mathrm{SU}(5)=\mathrm{Sp}(6) / \mathrm{Sp}(4)=S^{11}$ or $\operatorname{Spin}(7) / \operatorname{Spin}(5)=\mathrm{UT}\left(S^{6}\right)$, or else $G_{1}$ is the exceptional group $G_{2}$ and $H_{1}$ is $\mathrm{SU}(2)$ acting nontrivially on $G_{1}$, perhaps on both sides.

Since $M=G / H$ is homotopy equivalent to $\mathbf{H P}^{4} \# \mathbf{C a} \mathbf{P}^{2}$, it has the same Stiefel-Whitney classes as $\mathbf{H P}^{4} \# \mathbf{C a P}^{2}$, by Wu ([19], Theorem 11.14). In particular, the sphere $S^{8}$ in $\mathbf{H P}^{4} \# \mathbf{C a} \mathbf{P}^{2}$ has self-intersection number 1 , and so $w_{8} M$ is not in the subgroup $H^{4}\left(M, \mathbf{F}_{2}\right)^{2}$ of $H^{8}\left(M, \mathbf{F}_{2}\right)$. The biquotient $N:=\left(G_{1} \times G_{2}\right) /\left(H_{1} \times H_{2}\right)$ is a principal SU(2)-bundle over $M$, so its stable tangent bundle is the pullback of that of $M$. It follows that $w_{8} N \in H^{8}\left(N, \mathbf{F}_{2}\right)$ is not zero.

Suppose that $G_{1}$ is the exceptional group $G_{2}$. Then $M$ is a biquotient of the form $\left(G_{2} \times \operatorname{Spin}(9)\right) /(\mathrm{SU}(2) \times \operatorname{Spin}(8) \times \mathrm{SU}(2))$. Here $\operatorname{Spin}(8)$ must act trivially on $G_{2}$, and so we can write

$$
M=\left(G_{2} \times S^{8}\right) / \mathrm{SU}(2)^{2} .
$$

Any torus acting by isometries on an even-dimensional sphere has a fixed point, since every orthogonal representation of a torus is a sum of 2-dimensional representations and trivial representations. In particular, a maximal torus in $\mathrm{SU}(2)^{2}$ has a fixed point on $S^{8}$. Since $\mathrm{SU}(2)^{2}$ acts freely on $G_{2} \times S^{8}$, this torus acts freely on $G_{2}$. Since every element of $\mathrm{SU}(2)^{2}$ is conjugate to an element of the torus, $\mathrm{SU}(2)^{2}$ acts freely on $G_{2}$. Thus $M$ is a fiber bundle

$$
S^{8} \rightarrow M \rightarrow G_{2} / \mathrm{SU}(2)^{2}
$$

Since $S^{8}$ has signature zero, it follows that $M$ has signature zero, contradicting the fact that $M$ is homotopy equivalent to $\mathbf{H P}^{4} \# \mathbf{C a P} \mathbf{P}^{2}$. Thus the first factor $G_{1}$ is not the exceptional group $G_{2}$.

So $G_{1} / H_{1}$ is either $\operatorname{Spin}(12) / \operatorname{Spin}(11)=\mathrm{SU}(6) / \mathrm{SU}(5)=$ $\operatorname{Sp}(6) / \operatorname{Sp}(4)=S^{11}$ or $\operatorname{Spin}(7) / \operatorname{Spin}(5)=\mathrm{UT}\left(S^{6}\right)$, and we know that $G_{2} / H_{2}$ is $\operatorname{Spin}(9) / \operatorname{Spin}(8)=S^{8}$. But since $w_{8} N$ is not zero, $N$ is not an $S^{8}$-bundle over $S^{11}$ or over $\mathrm{UT}\left(S^{6}\right)$. So $H_{2}=\operatorname{Spin}(8)$ must act nontrivially on $G_{1}$. It follows that $G_{1} / H_{1}$ must be $\operatorname{Spin}(12) / \operatorname{Spin}(11)=S^{11}$. 
Thus $M$ is a biquotient of the form

$$
(\operatorname{Spin}(12) \times \operatorname{Spin}(9)) /(\operatorname{Spin}(11) \times \operatorname{Spin}(8) \times \mathrm{SU}(2)) .
$$

Here Spin(11) automatically acts trivially on $\operatorname{Spin}(9)$. So the biquotient

$$
N:=(\operatorname{Spin}(12) \times \operatorname{Spin}(9)) /(\operatorname{Spin}(11) \times \operatorname{Spin}(8))
$$

is completely determined by the homomorphism $\operatorname{Spin}(8) \rightarrow \operatorname{Spin}(12)$, which we know is nontrivial. (Since $\operatorname{Spin}(11)$ has finite centralizer on $\operatorname{Spin}(12)$, the action of $\operatorname{Spin}(8)$ on $\operatorname{Spin}(12)$ must be on the other side of $\operatorname{Spin}(12)$ from $\operatorname{Spin}(11)$.) There are 3 conjugacy classes of nontrivial homomorphisms $\operatorname{Spin}(8) \rightarrow \operatorname{Spin}(12)$, each of the form $W \oplus \mathbf{R}^{4}$, where the 8-dimensional real representation $W$ of $\operatorname{Spin}(8)$ is either the standard representation $V$ or one of the two spin representations. Thus $N$ is the $S^{11}$-bundle $S\left(W \oplus \mathbf{R}^{4}\right)$ over $\operatorname{Spin}(9) / \operatorname{Spin}(8)=S^{8}$. If $W$ is the standard representation $V$ of $\operatorname{Spin}(8)$, then the associated rank-8 vector bundle over $S^{8}$ has $w_{8}=0$, and so the manifold $N$ would have $w_{8} N=0$, contradicting what we know. So $W$ must be one of the two spin representations of $\operatorname{Spin}(8)$. If necessary, we can apply an order2 automorphism to $\operatorname{Spin}(8)$, not changing the conjugacy class of the standard inclusion $\operatorname{Spin}(8) \rightarrow \operatorname{Spin}(9)$, to arrange that $W=S^{-}$.

Thus $N$ is the $S^{11}$-bundle $S\left(S^{-} \oplus \mathbf{R}^{4}\right)$ over $S^{8}$. The action of $\mathrm{SU}(2)$ on $N$ is given by a homomorphism $\mathrm{SU}(2) \rightarrow \operatorname{Spin}(9)$ together with a homomorphism SU(2) $\rightarrow \operatorname{Spin}(4)$, because $\operatorname{Spin}(4)$ is the identity component of the centralizer of $\operatorname{Spin}(8)$ in $\operatorname{Spin}(12)$. The homomorphism $\mathrm{SU}(2) \rightarrow \operatorname{Spin}(9)$ determines the action of $\mathrm{SU}(2)$ on $S^{8}$ and on the Spin(9)-equivariant vector bundle $S^{-}$over $S^{8}$, while the homomorphism $\mathrm{SU}(2) \rightarrow \operatorname{Spin}(4)$ determines the action of $\mathrm{SU}(2)$ on $\mathbf{R}^{4}$. Since $\mathrm{SU}(2)$ acts freely on $N=S\left(S^{-} \oplus \mathbf{R}^{4}\right)$, it must act freely on $S\left(S^{-}\right)=S^{15}$ and on $S\left(\mathbf{R}^{4}\right)=S^{3} \times S^{8}$.

As in the proof that $\mathbf{C P}^{8} \# \mathbf{C} \mathbf{a P}^{2}$ is not homotopy equivalent to a biquotient, we compute that there is only one free $\mathrm{SU}(2)$-action on $S\left(S^{-} \oplus \mathbf{R}^{4}\right): \mathrm{SU}(2)$ must act by $\mathrm{SU}(2) \cong \operatorname{Spin}(3) \subset \operatorname{Spin}(9)$ on $S^{8}$ and on the bundle $S^{-}$over $S^{8}$, and by the standard representation $V_{\mathbf{R}}$ on $\mathbf{R}^{4}$. Thus we have described the manifold $M$ uniquely up to diffeomorphism. But we showed in Section 2 that exactly this manifold is diffeomorphic to $\mathbf{H P}^{4} \#-\mathbf{C a P}^{2}$. In particular, it is not homotopy equivalent to $\mathbf{H P}^{4} \# \mathbf{C a P} \mathbf{P}^{2}$. This completes the proof that $\mathbf{H} \mathbf{P}^{4} \# \mathbf{C a} \mathbf{P}^{2}$ is not homotopy equivalent to a biquotient. 


\section{The Cheeger manifold $\mathrm{CP}^{4 e} \# \mathrm{HP}^{2 e}$ is not homotopy equivalent to a biquotient}

Finally, we will show that $\mathbf{C P}^{4 e} \# \mathbf{H P}^{2 e}, e \geq 1$, is not homotopy equivalent to a biquotient $M=G / K$, completing the proof of Theorem 2.1.

The cohomology ring of $M$ is a complete intersection ring. The degrees of its generators and relations determine the rational homotopy groups of $M$. The result is that $G$ contributes degrees 3 and $4 e$ to $M$ and $K$ contributes degrees 1 and 2, where the 1 means that the abelianization of $K$ is isomorphic to $S^{1}$. Let $H$ be the commutator subgroup of $K$, and let $N$ be the biquotient $G / H$; then $M=N / S^{1}$. Here $G$ contributes degrees 3 and $4 e$ to $N$, while $H$ contributes degree 2. From the degree 2, it follows that $H$ has one more simple factor than $G$ has. Also, by Theorem 4.8, each simple factor of $G$ contributes at least one degree to $N$. So $G$ has at most two simple factors.

Furthermore, since $M$ and $\mathbf{C P}^{4 e} \# \mathbf{H} \mathbf{P}^{2 e}$ are homotopy equivalent, they have the same Stiefel-Whitney classes, by Wu ([19], Theorem 11.14). In particular, $w_{4} M$ is not in the subgroup $H^{2}\left(M, \mathbf{F}_{2}\right)^{2}$ of $H^{4}(M$, $\mathbf{F}_{2}$ ). Since $N$ is an $S^{1}$-bundle over $M$, the stable tangent bundle of $N$ is the pullback of that of $M$, and so $w_{4} N$ is not zero.

Suppose first that $G$ is simple. Since $H$ has one more simple factor than $G, H$ has two simple factors. We know that $G$ contributes degrees 3 and $4 e$ to $N$, and nothing else. In Theorem 4.8, cases (2), (3), (4) are incompatible with these degrees, and so case (1) must hold, that is, $G$ contributes its maximal degree to $N$. Thus $G$ has maximal degree 4e. The only simple groups which have 3 as a degree are the groups $\mathrm{SU}(n), n \geq 3$, and so $G$ is isomorphic to $\mathrm{SU}(4 e)$. Since $G$ has exactly one degree $4 e$, the known degrees of $N$ imply that all simple factors of $H$ have maximal degree less than $4 e$.

Suppose that $e \geq 2$. Then the second-largest degree, $4 e-1$, of $G=\mathrm{SU}(4 e)$ is killed by $H$. Let $H_{1}$ be a simple factor of $H$ which kills the degree $4 e-1$ of $G$. We know that $H_{1}$ has maximal degree less than $4 e$, and so it has maximal degree $4 e-1$, which implies that $H_{1}$ is isomorphic to $\mathrm{SU}(4 e-1)$. Any nontrivial homomorphism $\mathrm{SU}(4 e-1) \rightarrow \mathrm{SU}(4 e)$ has Dynkin index 1 , and so $H_{1}$ must act nontrivially on only one side of $G$; otherwise $\mathrm{SU}(4 e)$ would contribute degree 2 to $N$. So $G / H_{1}$ is isomorphic to the homogeneous space $\mathrm{SU}(4 e) / \mathrm{SU}(4 e-1)=S^{8 e-1}$. But then $H_{1}$ kills the degree 3 of $G$, which should appear in $M$. Thus $e \geq 2$ leads to a contradiction, for $G$ simple. 
This leaves the case $e=1$, with $G$ simple. As shown above, $G$ is isomorphic to $\mathrm{SU}(4)$. Since $G$ has degrees 2,3,4, the known degrees of $N$ imply that $H$ has degrees 2,2 . So $H$ is isomorphic to $\mathrm{SU}(2)^{2}$. Thus $N$ is a biquotient $\mathrm{SU}(4) / \mathrm{SU}(2)^{2}$. Indeed, there is at least one such biquotient, the homogeneous space $\mathrm{UT}\left(S^{5}\right)=\operatorname{Spin}(6) / \operatorname{Spin}(4)$, which admits a free $S^{1}$-action. In that case, the quotient is the 4-dimensional complex quadric $Q_{\mathbf{C}}^{4}$, which has the rational homotopy type of $\mathbf{C P}^{4} \# \mathbf{H P}^{2}$. But no biquotient $N=\mathrm{SU}(4) / \mathrm{SU}(2)^{2}$ can have the homotopy type of an $S^{1}$-bundle over $\mathbf{C P}^{4} \# \mathbf{H} \mathbf{P}^{2}$, by the following argument. By Singhof's description of the tangent bundle of a biquotient [25], as used in the previous section, we have

$$
w_{4} N=w_{4}(\mathrm{su}(4))-w_{4}\left(\mathrm{su}(2)_{1}\right)-w_{4}\left(\operatorname{su}(2)_{2}\right) .
$$

We then make the convenient calculation that

$$
w_{4}(\operatorname{su}(n))=0 \in H^{4}\left(\operatorname{BSU}(n), \mathbf{F}_{2}\right)
$$

for all even $n$. So $w_{4} N=0$, which contradicts what we know about $N$. Thus we have a contradiction from the assumption that $G$ is simple.

Thus $G$ has two simple factors. We return to the general case, $e \geq 1$. Since $H$ has one more simple factor than $G, H$ has three simple factors. Each simple factor of $G$ contributes exactly one degree to $N$. We can write $G=G_{1} \times G_{2}$ such that $G_{1}$ contributes degree 3 to $N$ and $G_{2}$ contributes degree $4 e$, and nothing more. By Theorems 4.8 and 5.3 applied to $G_{1}$, there is a simple factor $H_{1}$ of $H$ such that either $G_{1} / H_{1}$ is isomorphic to the homogeneous space $\mathrm{SU}(4) / \mathrm{Sp}(4)=S^{5}$ (which is the same as $\operatorname{Spin}(6) / \operatorname{Spin}(5))$ or $\left(G_{1}, H_{1}\right)$ is $(\mathrm{SU}(3), \mathrm{SU}(2))$ for some nontrivial action of $\mathrm{SU}(2)$ on $\mathrm{SU}(3)$.

If $\left(G_{1}, H_{1}\right)$ is $(\mathrm{SU}(3), \mathrm{SU}(2))$ and $\mathrm{SU}(2)$ acts nontrivially on both sides of $\mathrm{SU}(3)$, then the centralizers of both homomorphisms $\mathrm{SU}(2) \rightarrow$ $\mathrm{SU}(3)$ are finite or finite by $S^{1}$, so no other simple factor of $H$ can act on $G_{1}$. Moreover, the two nontrivial homomorphisms $\mathrm{SU}(2) \rightarrow \mathrm{SU}(3)$ have Dynkin indices 1 and 4 , by Lemma 5.2. So the map $\pi_{3} H_{1} \rightarrow \pi_{3} G_{1}$, $\mathbf{Z} \rightarrow \mathbf{Z}$, is either zero or multiplication by $1-4=-3$ or $4-1=3$. In any case, it is not surjective, which contradicts the fact that $\pi_{3} H \rightarrow \pi_{3} G_{1}$ must be surjective, since $\pi_{3} M=0$. So any $\mathrm{SU}(2)$ factor of $H$ acts nontrivially on at most on one side of $\mathrm{SU}(3)$. Moreover, $\mathrm{SU}(2)$ factors of $H$ are the only ones that can act on $G_{1}=\mathrm{SU}(3)$ (SU(3) factors of $H$ are excluded by Lemma 4.1). At least one $\mathrm{SU}(2)$ factor must act with Dynkin index 1 rather than 4 , again using that $\pi_{3} H \rightarrow \pi_{3} G_{1}$ is 
surjective. Thus, if $G_{1}$ is $\mathrm{SU}(3)$, then we can choose the simple factor $H_{1}$ isomorphic to $\mathrm{SU}(2)$ such that $G_{1} / H_{1}$ is isomorphic to the homogeneous space $\mathrm{SU}(3) / \mathrm{SU}(2)=S^{5}$.

Thus, $G_{1} / H_{1}$ is either $\operatorname{Spin}(6) / \operatorname{Spin}(5)=S^{5}$ or $\mathrm{SU}(3) / \mathrm{SU}(2)=S^{5}$. In both cases, $G_{1} / H_{1}$ adds degree 3 to $N$ and nothing else. Next, we can apply Theorems 4.8 and 5.3 to the second factor $G_{2}$, giving a simple factor $H_{2}$ with certain properties. From the known degrees of $N=$ $\left(G_{1} \times G_{2}\right) /\left(H_{1} \times H_{2} \times H_{3}\right)$, since $G_{1} / H_{1}$ adds degree 3 to $N$ and nothing else, $G_{2} / H_{2}$ cannot have any degrees with multiplicity $<0$ (that is, any degrees which occur in $H_{2}$ more than in $G_{2}$ ). Given this, Theorems 4.8 and 5.3 imply that either $e=1$ and $G_{2}$ is isomorphic to $\operatorname{Sp}(4)$, or there is a simple factor $H_{2}$ of $H$ such that $G_{2} / H_{2}$ is isomorphic to a homogeneous space $\operatorname{Spin}(8 e) / \operatorname{Spin}(8 e-1)=\mathrm{SU}(4 e) / \mathrm{SU}(4 e-1)=\mathrm{Sp}(4 e) / \mathrm{Sp}(4 e-2)=$ $S^{8 e-1}, \operatorname{Spin}(7) / G_{2}=S^{7}$ with $e=1, \operatorname{Spin}(9) / \operatorname{Spin}(7)=S^{15}$ with $e=2$, $\operatorname{Spin}(4 e+1) / \operatorname{Spin}(4 e-1)=\mathrm{UT}\left(S^{4 e}\right)$ with $e \geq 2, \operatorname{Spin}(8) / G_{2}=S^{7} \times S^{7}$ with $e=1, \operatorname{Spin}(9) / G_{2}$ with $e=2, \operatorname{Spin}(4 e+2) / \operatorname{Spin}(4 e-1)$ with $e \geq 2$, $\operatorname{Spin}(10) / \operatorname{Spin}(7)$ via the spin representation with $e=2$, or $\operatorname{Spin}(10) / G_{2}$ with $e=2$. In these homogeneous spaces, it is clear that the simple factor $H_{2}$ of $H$ is different from $H_{1}$, which is $\mathrm{SU}(2)$ or $\operatorname{Spin}(5)=\mathrm{Sp}(4)$.

When $G_{2} / H_{2}$ is one of the homogeneous spaces diffeomorphic to $S^{8 e-1}$ or $\operatorname{UT}\left(S^{4 e}\right)$, then $G_{2} / H_{2}$ adds only one degree $4 e$ to $N$ and nothing else. In those cases, the third factor $H_{3}$ of $H$ has degree 2 only, so $H_{3}$ is isomorphic to $\mathrm{SU}(2)$. When $G_{2}$ is isomorphic to $\operatorname{Sp}(4)$, the product of the two simple factors of $H$ besides $H_{1}, H_{2} \times H_{3}$, has degrees 2, 2, and so $H_{2}$ and $H_{3}$ are both isomorphic to $\mathrm{SU}(2)$. When $G_{2} / H_{2}$ is $\operatorname{Spin}(8) / G_{2}=S^{7} \times S^{7}$ with $e=1$ or $\operatorname{Spin}(9) / G_{2}$ with $e=2$, then $H_{3}$ has degrees 2,4 , and so $H_{3}$ is isomorphic to $\mathrm{Sp}(4)$. Finally, the cases where $G_{2} / H_{2}$ is $\operatorname{Spin}(4 e+2) / \operatorname{Spin}(4 e-1)$ with $e \geq 2, \operatorname{Spin}(10) / \operatorname{Spin}(7)$ with $e=2$, or $\operatorname{Spin}(10) / G_{2}$ with $e=2$ cannot occur. In these cases, part (4) of Theorem 5.3 implies that $H$ must have a factor $\mathrm{SU}(2 e+1)$, which must be the third factor $H_{3}$. But then $H$ has one more degree 3 than $G$, contradicting the known degrees of $N=G / H$.

The cases where $H_{3}$ is $\mathrm{Sp}(4)$ are easier to analyze, so we consider them first. Either $G_{2} / H_{2}$ is $\operatorname{Spin}(8) / G_{2}=S^{7} \times S^{7}$ and $e=1$, or $G_{2} / H_{2}$ is $\operatorname{Spin}(9) / G_{2}$ and $e=2$. We know that $G_{1} / H_{1}$ is either $\mathrm{SU}(3) / \mathrm{SU}(2)=$ $S^{5}$ or $\operatorname{Spin}(6) / \operatorname{Spin}(5)=S^{5}$. By the known low-dimensional representations of $H_{3}=\mathrm{Sp}(4)$, the action of $H_{3}$ on $G_{1} / H_{1}=S^{5}$ must have a fixed point. So there is a subgroup of $H_{1} \times H_{3}$ which projects isomorphically to $H_{3}=\mathrm{Sp}(4)$ and which fixes a point in $G_{1}$. Also, the exceptional group $G_{2}$ must act trivially on $G_{1}$ (which is $\operatorname{Spin}(6)$ or $\mathrm{SU}(3)$ ), so we 
have a subgroup of $H$ isomorphic to $\operatorname{Sp}(4) \times G_{2}$ which fixes a point in $G_{1}$. Since $H$ acts freely on $G$, we have a free action of $\operatorname{Sp}(4) \times G_{2}$ on the second factor $\operatorname{Spin}(8)$ or $\operatorname{Spin}(9)$. We compute, however, that there is no such free action.

So we must have $H_{3}=\mathrm{SU}(2)$. We know that $G_{1} / H_{1}$ is either $\mathrm{SU}(3) / \mathrm{SU}(2)=S^{5}$ or $\operatorname{Spin}(6) / \operatorname{Spin}(5)=S^{5}$. Also, either $e=1, G_{2}$ is isomorphic to $\mathrm{Sp}(4)$ and $H_{2}$ is isomorphic to $\mathrm{SU}(2)$, or $G_{2} / H_{2}$ is a homogeneous space $\operatorname{Spin}(8 e) / \operatorname{Spin}(8 e-1)=\mathrm{SU}(4 e) / \mathrm{SU}(4 e-1)=$ $\operatorname{Sp}(4 e) / \operatorname{Sp}(4 e-2)=S^{8 e-1}, \operatorname{Spin}(7) / G_{2}=S^{7}$ with $e=1, \operatorname{Spin}(9) / \operatorname{Spin}(7)$ $=S^{15}$ with $e=2$, or $\operatorname{Spin}(4 e+1) / \operatorname{Spin}(4 e-1)=\mathrm{UT}\left(S^{4 e}\right)$ with $e \geq 2$.

We begin with the case where $G_{1} / H_{1}$ is $\mathrm{SU}(3) / \mathrm{SU}(2)=S^{5}$ and $G_{2} / H_{2}$ is $\operatorname{Spin}(8 e) / \operatorname{Spin}(8 e-1)=S^{8 e-1}$. This turns out to be the main step of the whole proof; most other cases will reduce to this one. The group $H_{2}=\operatorname{Spin}(8 e-1)$ must act trivially on $G_{1}=\mathrm{SU}(3)$, and so $\left(G_{1} \times G_{2}\right) /\left(H_{1} \times H_{2}\right)$ is an $S^{8 e-1}$-bundle over $S^{5}$. The manifold $M$ is the quotient of this bundle by a free action of a group $D:=K /\left(H_{1} \times H_{2}\right)$ which is isogenous to $S^{1} \times \mathrm{SU}(2)$. Since $\pi_{2} M$ is isomorphic to $\mathbf{Z}, \pi_{1} D$ is isomorphic to $\mathbf{Z}$. So $D$ is isomorphic to $S^{1} \times \mathrm{SU}(2)$ or to $\mathrm{U}(2)$.

In dimensions less than $8 e-1, M$ clearly has the homotopy type of a homotopy quotient $S^{5} / / D$, or equivalently an $S^{5}$-bundle over the classifying space $\mathrm{B} D$. Here $D$ acts on $S^{5}$ through the group U(3), coming from the group $G_{1}=\mathrm{SU}(3)$ together with the centralizer $S^{1}$ of $H_{1}=\mathrm{SU}(2)$ in $G_{1}$. From the cohomology ring of $M$, the Euler class of the homomorphism $D \rightarrow \mathrm{U}(3)$ must be the product of a generator of $H^{2}(\mathrm{~B} D, \mathbf{Z})$ with an element of $H^{4}(\mathrm{~B} D, \mathbf{Z})$ that generates $H^{4} /\left(H^{2}\right)^{2}$. Let $L$ be the standard 1-dimensional complex representation of $S^{1}, V$ be the standard 2dimensional representation of $\mathrm{SU}(2)$, and $E$ the standard 2-dimensional representation of $\mathrm{U}(2)$. By inspecting the low-dimensional representations of $D$, it follows that the homomorphism $D \rightarrow \mathrm{U}(3)$ is isomorphic to $L^{ \pm 1} \oplus L^{a} \otimes V$ if $D$ is $S^{1} \times \mathrm{SU}(2)$, or to $(\operatorname{det} E)^{ \pm 1} \oplus(\operatorname{det} E)^{a} \otimes E$ if $D$ is $\mathrm{U}(2)$, for some sign and some integer $a$. In particular, the subgroup $\mathrm{SU}(2)=H_{3}$ of $D$ acts on $G_{1} / H_{1}=\mathrm{SU}(3) / \mathrm{SU}(2)=S^{5}$ by the natural inclusion $\mathrm{SU}(2) \rightarrow \mathrm{SU}(3)$, on the other side of $G_{1}$ from $H_{1}$.

It follows that the diagonal subgroup $C$ in $H_{1} \times H_{3}=\mathrm{SU}(2) \times \mathrm{SU}(2)$ has a fixed point in $\mathrm{SU}(3)$. So $C$ must act freely on $S^{8 e-1}$. It follows that $C$ acts on $S^{8 e-1}$ by the real representation $\left(V_{\mathbf{R}}\right)^{2 e}$, where $V$ is the standard 2-dimensional complex representation of $C=\mathrm{SU}(2)$. In particular, the associated complex representation is a direct sum of copies of $V$. By the Clebsch-Gordan formula, an irreducible complex representation of $\mathrm{SU}(2) \times \mathrm{SU}(2)$ whose restriction to the diagonal subgroup 
is a sum of copies of $V$ must be isomorphic to $V_{1} \otimes \mathbf{C}$ or $\mathbf{C} \otimes V_{2}$, the standard representations of the two factors. Therefore the action of $H_{1} \times H_{3}=\mathrm{SU}(2) \times \mathrm{SU}(2)$ on $S^{8 e-1}$ must be given by the real representation associated to the complex representation $\left(V_{1}\right)^{\oplus j} \oplus\left(V_{3}\right)^{\oplus 2 e-j}$ for some $0 \leq j \leq 2 e$.

The manifold $M$ can be written

$$
M=\left(\mathrm{SU}(3) \times S^{8 e-1}\right) /\left(\left(\mathrm{SU}(2)^{2} \times \mathbf{R}\right) / \mathbf{Z}\right) .
$$

Here the subgroup $\mathbf{Z}$ of $\mathrm{SU}(2)^{2} \times \mathbf{R}$ is generated by an element of the form $( \pm 1, \pm 1,1)$, which we write as $\left(e\left(a_{0}\right), e\left(b_{0}\right), 1\right)$ for $a_{0}, b_{0} \in\{0,1 / 2\}$, where $e(t):=e^{2 \pi i t}$. The group $\left(\mathrm{SU}(2)^{2} \times \mathbf{R}\right) / \mathbf{Z}$ acts on $\mathrm{SU}(3) \times S^{8 e-1}$ via homomorphisms to $\mathrm{SU}(3)^{2} / Z(\mathrm{SU}(3))$ and to $\mathrm{SO}(8 e)$.

We can assume that the first factor $\mathrm{SU}(2)$ of $\left(\mathrm{SU}(2)^{2} \times \mathbf{R}\right) / \mathbf{Z}$ acts on $\mathrm{SU}(3)$ by the standard inclusion on the left, while the second factor $\mathrm{SU}(2)$ acts on $\mathrm{SU}(3)$ by the standard inclusion on the right. So the two homomorphisms from $\mathbf{R}$ to $\mathrm{SU}(3)$ both map into the centralizer of $\mathrm{SU}(2)$ in $\mathrm{SU}(3)$. That is, they map $t \in \mathbf{R}$ to the diagonal matrices

$$
(e(a t), e(a t), e(-2 a t)),(e(b t), e(b t), e(-2 b t))
$$

for some $a, b$. Since the generator of the above subgroup $\mathbf{Z}$ in $\mathrm{SU}(2)^{2} \times \mathbf{R}$ must map into $Z(\mathrm{SU}(3)) \cong \mathbf{Z} / 3 \subset \mathrm{SU}(3)^{2}, a$ and $b$ must satisfy:

$$
\begin{aligned}
a+a_{0} & \in \frac{1}{3} \mathbf{Z} \\
b+b_{0} & \in \frac{1}{3} \mathbf{Z} \\
a+a_{0} & \equiv b+b_{0} \quad(\bmod \mathbf{Z}) .
\end{aligned}
$$

The homomorphism $\mathrm{SU}(2)^{2} \rightarrow \mathrm{SO}(8 e)$ is by the real representation $\left(V_{1}\right)^{\oplus j} \oplus\left(V_{2}\right)^{\oplus 2 e-j}$ for some $0 \leq j \leq 2 e$. The centralizer of this homomorphism has identity component $\operatorname{Sp}(2 j) \times \operatorname{Sp}(4 e-2 j)$, whose maximal torus is conjugate to the center $\left(S^{1}\right)^{2 e}$ of $\mathrm{U}(2)^{2 e}$. So we can assume that $\mathbf{R}$ maps into this center. Thus the homomorphism from $\left(\mathrm{SU}(2)^{2} \times \mathbf{R}\right) / \mathbf{Z}$ to $\mathrm{SO}(8 e)$ is the direct sum of $2 e$ homomorphisms to $\mathrm{U}(2)$, of the form $(A, B, t) \mapsto e\left(c_{i} t\right) A$ for $1 \leq i \leq j$ and $(A, B, t) \mapsto e\left(d_{i} t\right) B$ for $j+1 \leq i \leq 2 e$. Since this homomorphism is trivial on the subgroup $\mathbf{Z}$, the numbers $c_{i}$ and $d_{i}$ must satisfy $c_{i}+a_{0} \in \mathbf{Z}$ and $d_{i}+b_{0} \in \mathbf{Z}$.

The action of $\left(\mathrm{SU}(2)^{2} \times \mathbf{R}\right) / \mathbf{Z}$ on $\mathrm{SU}(3) \times S^{8 e-1}$ is free. We compute 
that this means that, for all $1 \leq i \leq j$,

$$
\begin{gathered}
a+2 b-c_{i}= \pm 1 \\
a+2 b+c_{i}= \pm 1 \\
-2 a+2 b= \pm 1
\end{gathered}
$$

and for all $j+1 \leq i \leq 2 e$,

$$
\begin{gathered}
2 a+b-d_{i}= \pm 1 \\
2 a+b+d_{i}= \pm 1 \\
-2 a+2 b= \pm 1
\end{gathered}
$$

If $j \geq 1$, then the first two equations imply that either $a+2 b=0$ and $c_{i}= \pm 1$, or $a+2 b= \pm 1$ and $c_{i}=0$, for $1 \leq i \leq j$. In particular, $c_{i}$ is an integer, which implies (since $c_{i}+a_{0} \in \mathbf{Z}$ ) that $a_{0}$ is 0 , not $1 / 2$. Since $a+a_{0} \in(1 / 3) \mathbf{Z}$, it follows in particular that $a$ is 2-integral; since $-2 a+2 b= \pm 1, b$ is not 2-integral. Therefore $b_{0}$ is $1 / 2$, not 0 . But if $j \leq 2 e-1$, then we would get the opposite conclusion (that $a_{0}=1 / 2$ and $b_{0}=1$ ) from the second three equations above. So we must have either $j=0$ or $j=2 e$. After switching the two $\mathrm{SU}(2)$ factors if necessary, we can assume that $j=2 e$. That is, $\mathrm{SU}(2)^{2}$ acts on $S^{8 e-1}$ by the real representation $\left(V_{1}\right)^{2 e}$. Also, we have $a_{0}=0$ and $b=1 / 2$, which means that the subgroup $\mathbf{Z}$ of $\mathrm{SU}(2)^{2} \times \mathbf{R}$ is generated by $(1,-1,1 \in \mathbf{R})$.

Since the second factor $\mathrm{SU}(2)$ acts only on $\mathrm{SU}(3)$, we can rewrite $M$ as

$$
M=\left(S^{5} \times S^{8 e-1}\right) /\left(\mathrm{SU}(2) \times S^{1}\right) .
$$

Here we have used that the quotient of $\left(\mathrm{SU}(2)^{2} \times \mathbf{R}\right) / \mathbf{Z}$ by the second copy of $\mathrm{SU}(2)$ is isomorphic to $\mathrm{SU}(2) \times S^{1}$. The action of $\mathrm{SU}(2) \times S^{1}$ on $S^{8 e-1}$ is given by the complex representation $\oplus_{i=1}^{2 e} V \otimes L^{c_{i}}$. Also, we compute that the action of $\mathrm{SU}(2) \times S^{1}$ on $S^{5}$ is by the complex representation $\left(V \otimes L^{a+2 b}\right) \oplus L^{-2 a+2 b}$. As computed above, $-2 a+2 b=$ \pm 1 . And either $a+2 b= \pm 1$ and $c_{i}=0$ for all $i$, or $a+2 b=0$ and $c_{i}= \pm 1$ for all $i$.

In the first case, where $c_{i}=0$, we can conjugate the action of $\mathrm{SU}(2) \times$ $S^{1}$ on $S^{5}$ in the orthogonal group $\mathrm{O}(6)$ to make $a+2 b$ and $-2 a+2 b$ equal to 1 , rather than -1 . Thus $M$ is the manifold

$$
\left(S((V \otimes L) \oplus L) \times S\left(V^{\oplus 2 e}\right)\right) /\left(\mathrm{SU}(2) \times S^{1}\right) .
$$

So $M$ is a $\mathbf{C P}^{2}$-bundle over $\mathbf{H} \mathbf{P}^{2 e-1}$, and so $M$ has signature zero, contradicting that $M$ is homotopy equivalent to $\mathbf{C P}^{4 e} \# \mathbf{H} \mathbf{P}^{2 e}$. In fact, 
this $\mathbf{C} \mathbf{P}^{2}$-bundle over $\mathbf{H} \mathbf{P}^{2 e-1}$ is the one diffeomorphic to $\mathbf{C P}^{4 e} \#-$ $\mathbf{H P}^{2 e}$, as mentioned in Section 2. In the second case, where $c_{i}= \pm 1$, we can conjugate the homomorphisms from $\mathrm{SU}(2) \times S^{1}$ to the orthogonal groups $\mathrm{O}(6)$ and $\mathrm{O}(8 e)$ to make $-2 a+2 b=1$ and $c_{i}=1$ for all $i$. Thus, $M$ is the manifold

$$
\left(S(V \oplus L) \times S\left((V \otimes L)^{\oplus 2 e}\right)\right) /\left(\mathrm{SU}(2) \times S^{1}\right) .
$$

Again, we showed in Section 2 that this manifold is diffeomorphic to $\mathbf{C P}{ }^{4 e} \#-\mathbf{H P}^{2 e}$. Thus it is not homotopy equivalent to $\mathbf{C P} \mathbf{P}^{4 e} \# \mathbf{H P}^{2 e}$. This completes the proof that we cannot have $G_{1} / H_{1}$ equal to $\mathrm{SU}(3) / \mathrm{SU}(2)=S^{5}$ and $G_{2} / H_{2}$ equal to $\operatorname{Spin}(8 e) / \operatorname{Spin}(8 e-1)=S^{8 e-1}$.

We next consider the case where $G_{1} / H_{1}$ is $\operatorname{Spin}(6) / \operatorname{Spin}(5)=S^{5}$ and $G_{2} / H_{2}$ is $\operatorname{Spin}(8 e) / \operatorname{Spin}(8 e-1)=S^{8 e-1}$. A first observation is that $\operatorname{Spin}(5)$ has finite centralizer in $\operatorname{Spin}(6)$, and so all factors of $K$ except $\operatorname{Spin}(5)$ act on the other side of $\operatorname{Spin}(6)$ from $\operatorname{Spin}(5)$. Likewise, all factors of $K$ except $\operatorname{Spin}(8 e-1)$ act on the other side of $\operatorname{Spin}(8 e)$ from $\operatorname{Spin}(8 e-1)$. Furthermore, $\operatorname{Spin}(8 e-1)$ must act trivially on $\operatorname{Spin}(6)$, and so $\left(G_{1} \times G_{2}\right) /\left(H_{1} \times H_{2}\right)$ is an $S^{8 e-1}$-bundle over $S^{5}$, which we can write as $\left(\operatorname{Spin}(6) \times S^{8 e-1}\right) / \operatorname{Spin}(5)$.

The homomorphism $K \rightarrow(G \times G) / Z(G)$ which defines the action of $K$ on $G$ gives a homomorphism from $D:=K /\left(H_{1} \times H_{2}\right)$ to $\mathrm{SO}(6)$. Here $D$ is isogenous to $\mathrm{SU}(2) \times S^{1}$ and has fundamental group isomorphic to $\mathbf{Z}$, so $D$ is isomorphic to $\mathrm{SU}(2) \times S^{1}$ or to $\mathrm{U}(2)$. In dimensions less than $8 e-1, M$ has the homotopy type of the homotopy quotient $S^{5} / / D$ defined by the homomorphism $D \rightarrow \mathrm{SO}(6)$, or equivalently of an $S^{5}$ bundle over the classifying space $\mathrm{B} D$. From the cohomology ring of $M$, the Euler class of the homomorphism $D \rightarrow \mathrm{SO}(6)$ must be the product of some generator of $H^{2}(\mathrm{~B} D, \mathbf{Z})$ with some element of $H^{4}(\mathrm{~B} D, \mathbf{Z})$ which generates $H^{4} /\left(H^{2}\right)^{2}$. By inspecting the low-dimensional real representations of $D$, it follows that the homomorphism $D \rightarrow \mathrm{SO}(6)$ is the real representation associated to a 3 -dimensional complex representation of $D$. For $D=S^{1} \times \mathrm{SU}(2)$, write $L$ for the standard 1-dimensional complex representation of $S^{1}$ and $V$ for the standard 2-dimensional complex representation of $\mathrm{SU}(2)$. In order to have an Euler class of the form above, the homomorphism $D \rightarrow \mathrm{SO}(6)$ must come from the complex representation $L^{ \pm 1}+L^{b} \otimes V$ for some sign and some integer $b$. The Euler class of this representation is $\pm x y$, where we let $x=c_{1} L$ and $y=c_{2} V+b^{2} x^{2}$. Likewise, for $D=\mathrm{U}(2)$, let $E$ be the standard 2-dimensional complex representation of $D$. In order to have an Euler class of the form above, 
the homomorphism $D \rightarrow \mathrm{SO}(6)$ must come from the complex representation $(\operatorname{det} E)^{ \pm 1}+(\operatorname{det} E)^{d} \otimes E$ for some sign and some integer $d$. The Euler class in $H^{6} \mathrm{BU}(2)$ of this representation is $\pm u v$, where we let $u=c_{1} E$ and $v=c_{2} E+\left(d^{2}+d\right) u^{2}$.

Because the homomorphism $D \rightarrow \mathrm{SO}(6)$ factors through $\mathrm{U}(3)$, we can replace $G_{1} / H_{1}=\operatorname{Spin}(6) / \operatorname{Spin}(5)=S^{5}$ by $G_{1} / H_{1}=\widetilde{\mathrm{U}}(3) / \widetilde{\mathrm{U}}(2)$, in our description of $M$ as a biquotient. Here $\widetilde{\mathrm{U}}(n)$ denotes the inverse image of $\mathrm{U}(n) \subset \mathrm{SO}(2 n)$ in $\operatorname{Spin}(2 n)$. We can then apply the proof of Lemma 3.1 to replace $\widetilde{U}(3)$ by $\mathrm{SU}(3)$. Thus we have reduced to the case where $G_{1} / H_{1}$ is $\mathrm{SU}(3) / \mathrm{SU}(2)=S^{5}$ and $G_{2} / H_{2}$ is $\operatorname{Spin}(8 e) / \operatorname{Spin}(8 e-1)=S^{8 e-1}$. But we have shown that the latter case cannot occur. This completes the proof that $G_{2} / H_{2}$ cannot be $\operatorname{Spin}(8 e) / \operatorname{Spin}(8 e-1)=S^{8 e-1}$, either when $G_{1} / H_{1}$ is $\operatorname{Spin}(6) / \operatorname{Spin}(5)$ or when it is $\mathrm{SU}(3) / \mathrm{SU}(2)$.

The situation now is as follows. First, we know that $G_{1} / H_{1}$ is either $\mathrm{SU}(3) / \mathrm{SU}(2)=S^{5}$ or $\operatorname{Spin}(6) / \operatorname{Spin}(5)=S^{5}$. Also, either $e=1, G_{2}$ is isomorphic to $\mathrm{Sp}(4)$ and $H_{2}$ is isomorphic to $\mathrm{SU}(2)$, or $G_{2} / H_{2}$ is a homogeneous space $\operatorname{Spin}(8 e) / \operatorname{Spin}(8 e-1)=\mathrm{SU}(4 e) / \mathrm{SU}(4 e-1)=$ $\operatorname{Sp}(4 e) / \operatorname{Sp}(4 e-2)=S^{8 e-1}, \operatorname{Spin}(7) / G_{2}=S^{7}$ with $e=1, \operatorname{Spin}(9) / \operatorname{Spin}(7)$ $=S^{15}$ with $e=2$, or $\operatorname{Spin}(4 e+1) / \operatorname{Spin}(4 e-1)=\mathrm{UT}\left(S^{4 e}\right)$ with $e \geq$ 2. We have shown that $G_{2} / H_{2}$ cannot be $\operatorname{Spin}(8 e) / \operatorname{Spin}(8 e-1)=$ $S^{8 e-1}$, either when $G_{1} / H_{1}$ is $\operatorname{Spin}(6) / \operatorname{Spin}(5)$ or when it is $\mathrm{SU}(3) / \mathrm{SU}(2)$. Most other cases reduce to this one. Namely, suppose that $G_{2} / H_{2}$ is diffeomorphic to a sphere $S^{8 e-1}$ (noting that in all these cases $G_{2} \times$ $Z_{G_{2}}\left(H_{2}\right)$ acts by isometries in the usual metric on the sphere), and that $H_{2}$ acts trivially on $G_{1}$. Then we can simply replace $G_{2} / H_{2}$ by $\operatorname{Spin}(8 e) / \operatorname{Spin}(8 e-1)=S^{8 e-1}$ without changing the biquotient $M$. Since $G_{1}$ is small, namely $\operatorname{Spin}(6)$ or $\mathrm{SU}(3)$, the hypothesis that $H_{2}$ acts trivially on $G_{1}$ is automatic for most of the pairs $G_{2} / H_{2}$. Namely this holds when there is no nontrivial homomorphism $H_{2} \rightarrow G_{1}$, or also when $H_{2}$ is isomorphic to $G_{1}$ by Lemma 4.1.

The cases not covered by this argument are: $G_{1} / H_{1}$ is $\mathrm{SU}(3) / \mathrm{SU}(2)$ $=S^{5}$ or $\operatorname{Spin}(6) / \operatorname{Spin}(5)=S^{5}, G_{2}$ is isomorphic to $\mathrm{Sp}(4)$, and $H_{2}$ is isomorphic to $\mathrm{SU}(2)$, where $e=1 ; G_{1} / H_{1}$ is $\operatorname{Spin}(6) / \operatorname{Spin}(5)$ and $G_{2} / H_{2}$ is $\mathrm{SU}(4) / \mathrm{SU}(3)=S^{15}$ with $H_{2}$ acting nontrivially on $G_{1}$, where $e=2$; or $G_{1} / H_{1}$ is $\mathrm{SU}(3) / \mathrm{SU}(2)$ or $\operatorname{Spin}(6) / \operatorname{Spin}(5)$ and $G_{2} / H_{2}$ is $\operatorname{Spin}(4 e+1) / \operatorname{Spin}(4 e-1)=\mathrm{UT}\left(S^{4 e}\right)$ with $e \geq 2$.

The last case, where $G_{2} / H_{2}$ is $\mathrm{UT}\left(S^{4 e}\right)$ with $e \geq 2$, is easy to exclude. Let $N=\left(G_{1} \times G_{2}\right) /\left(H_{1} \times H_{2} \times H_{3}\right)$, so that $M=N / S^{1}$. Because the groups involved in $N$ are simply connected, $N$ is 2-connected, and so 
$N$ is the $S^{1}$-bundle over $M$ corresponding to a generator of $H^{2}(M, \mathbf{Z})$. Since $M$ has the integral cohomology ring of $\mathbf{C P}^{4 e} \# \mathbf{H} \mathbf{P}^{2 e}$, the spectral sequence of this $S^{1}$-bundle shows that $N$ has the integral cohomology ring of $S^{5} \times \mathbf{H P}^{2 e-1}$. Next, let $Y$ be the manifold $\left(G_{1} \times G_{2}\right) /\left(H_{1} \times H_{2}\right)$, which is an $\mathrm{SU}(2)$-bundle over $N$ because $H_{3}=\mathrm{SU}(2)$. Because $H_{2}=$ $\operatorname{Spin}(4 e-1)$ and $e \geq 2, H_{2}$ acts trivially on $G_{1}$ (which is $\operatorname{Spin}(6)$ or $\mathrm{SU}(3))$, and so $Y$ is a $\mathrm{UT}\left(S^{4 e}\right)$-bundle over $S^{5}$. In particular, $Y$ is 4 connected. Also, the spectral sequence computing the cohomology of $Y$ collapses for degree reasons, and so $Y$ has 2-torsion in its cohomology because $\operatorname{UT}\left(S^{4 e}\right)$ does. But $Y$ is also an $S^{3}$-bundle over $N$, and because $Y$ is 4-connected, the Euler class of this bundle must be a generator of $H^{4}(N, \mathbf{Z})$. So the spectral sequence of this $S^{3}$-bundle shows that $Y$ has the integral cohomology ring of $S^{5} \times S^{8 e-1}$. This contradicts the fact that $Y$ has 2-torsion. So this case, $G_{2} / H_{2}=\mathrm{UT}\left(S^{4 e}\right)$ with $e \geq 2$, does not occur.

Next, we consider the case where $G_{1} / H_{1}$ is $\operatorname{Spin}(6) / \operatorname{Spin}(5)=S^{5}$ and $G_{2} / H_{2}$ is $\mathrm{SU}(4) / \mathrm{SU}(3)=S^{15}$, with $\mathrm{SU}(3)$ acting nontrivially on $\operatorname{Spin}(6)$. Here $e=2$. The point is that any nontrivial action of $\mathrm{SU}(3)$ on $S^{5}$ is isomorphic to the standard action, and hence is transitive. Thus $H$ acts transitively on the factor $G_{1}$ of $G$, contrary to Convention 3.4.

This completes the proof that $\mathbf{C P}^{4 e} \# \mathbf{H} \mathbf{P}^{2 e}$ is not homotopy equivalent to a biquotient for all $e \geq 2$. Ironically, the hardest case of all is the case $e=1$, that is, the proof that $\mathbf{C P}^{4} \# \mathbf{H} \mathbf{P}^{2}$ is not homotopy equivalent to a biquotient.

For $e=1$, it remains to consider the case where $G_{1} / H_{1}$ is either $\mathrm{SU}(3) / \mathrm{SU}(2)=S^{5}$ or $\operatorname{Spin}(6) / \operatorname{Spin}(5)=S^{5}, G_{2}$ is isomorphic to $\operatorname{Sp}(4)$, and $\mathrm{H}_{2}$ is isomorphic to $\mathrm{SU}(2)$. Thus the 9 -manifold $N$ is a biquotient of the form

$$
(\mathrm{SU}(3) \times \mathrm{Sp}(4)) / \mathrm{SU}(2)^{3}
$$

or

$$
(\operatorname{Spin}(6) \times \mathrm{Sp}(4)) /\left(\operatorname{Spin}(5) \times \mathrm{SU}(2)^{2}\right),
$$

where the first factor $H_{1}$ acts by the standard inclusion on one side of $G_{1}$ and trivially on the other side of $G_{1}$. Also, if $H_{2}$ or $H_{3}$ (each isomorphic to $\mathrm{SU}(2))$ acts trivially on $G_{1}$, and acts trivially on one side of $\operatorname{Sp}(4)$ and by the standard inclusion $V \oplus \mathbf{C}^{2}$ on the other, then we can replace the quotient $\operatorname{Sp}(4) / \mathrm{SU}(2)=S^{7}$ by $\operatorname{Spin}(8) / \operatorname{Spin}(7)=S^{7}$ and thus reduce to an earlier case. So we can assume that neither $\mathrm{H}_{2}$ nor $H_{3}$ has these properties. 
We checked earlier that the Stiefel-Whitney class $w_{4} N$ is not zero. It is convenient to observe now that the Pontrjagin class $p_{1}\left(\mathbf{H P}^{2}\right)$ is $2 z$, where $z$ is a generator of $H^{4}\left(\mathbf{H P}^{2}, \mathbf{Z}\right) \cong \mathbf{Z}$. Furthermore, Wu showed that the first Pontrjagin class of a closed manifold is a homotopy invariant modulo 12 [28]. Since $M$ is homotopy equivalent to $\mathbf{C P}^{4} \# \mathbf{H P}^{2}$, $p_{1} M$ in $H^{4} M /\left(H^{2} M\right)^{2} \cong \mathbf{Z}$ is 2 times the class of some generator, modulo 12. Therefore, the $S^{1}$-bundle $N$ over $M$ has $p_{1} N$ equal to 2 times the class of some generator of $H^{4} N \cong \mathbf{Z}$, modulo 12 .

Lemma 8.1. If $H_{1}$ acts trivially on $G_{2}=\mathrm{Sp}(4)$, then $H_{2} \times H_{3}=$ $\mathrm{SU}(2)^{2}$ does not act freely on $G_{2}=\operatorname{Sp}(4)$.

Proof. Suppose that $H_{1}$ acts trivially on $\mathrm{Sp}(4)$ and that $H_{2} \times H_{3}$ acts freely on $\mathrm{Sp}(4)$. Since $H_{1}$ acts trivially on $\mathrm{Sp}(4)$, we can enlarge $G_{1}$ and $H_{1}$ if necessary to make $G_{1} / H_{1}$ equal to $\operatorname{Spin}(6) / \operatorname{Spin}(5)$, rather than $\mathrm{SU}(3) / \mathrm{SU}(2)$. The quotient $\mathrm{Sp}(4) /\left(H_{2} \times H_{3}\right)$ is diffeomorphic to $S^{4}$, by Lemma 6.2. Also, $N$ is the $S^{5}$-bundle over $S^{4}$ associated to some homomorphism from $H_{2} \times H_{3}$ to $\operatorname{Spin}(6)$. By our knowledge of $p_{1} N$, $p_{1}$ of this $S^{5}$-bundle in $H^{4}\left(S^{4}, \mathbf{Z}\right) \cong \mathbf{Z}$ must be 2 times some generator, modulo 12 .

By Lemma 6.2, after switching $H_{2}$ and $H_{3}$ and switching the two sides of $\mathrm{Sp}(4)$ if necessary, $H_{2} \times H_{3}$ acts on $\mathrm{Sp}(4)$ by either $\left(V_{2} \oplus V_{3}, \mathbf{C}^{4}\right)$ or $\left(V_{2} \oplus \mathbf{C}^{2},\left(V_{3}\right)^{\oplus 2}\right)$. First suppose that $H_{2} \times H_{3}$ acts on $\operatorname{Sp}(4)$ by $\left(V_{2} \oplus V_{3}, \mathbf{C}^{4}\right)$. The conjugacy classes of homomorphisms from $\mathrm{H}_{2} \times \mathrm{H}_{3}$ to $\operatorname{Spin}(6)$ have complexifications: $\mathbf{C}^{6},\left(S^{2} V_{2}\right)^{\oplus 2}, S^{2} V_{2}+S^{2} V_{3},\left(S^{2} V_{3}\right)^{\oplus 2}$, $\left(V_{2}\right)^{\oplus 2} \oplus \mathbf{C}^{2},\left(V_{3}\right)^{\oplus 2} \oplus \mathbf{C}^{2}, V_{2} \otimes V_{3} \oplus \mathbf{C}^{2}, S^{4} V_{2} \oplus \mathbf{C}$, and $S^{4} V_{3} \oplus \mathbf{C}$. The given homomorphism from $\mathrm{H}_{2} \times \mathrm{H}_{3}$ to $\operatorname{Spin}(6)$ must have first Pontrjagin class (that is, $-c_{2}$ of the complexification) equal to 2 times some generator of $H^{4}\left(S^{4}, \mathbf{Z}\right)$ modulo 12 , where both $c_{2} V_{2}$ and $c_{2} V_{3}$ represent the same generator of $H^{4}\left(S^{4}, \mathbf{Z}\right)$. This only occurs when the complexification is $\left(V_{2}\right)^{\oplus 2} \oplus \mathbf{C}^{2}$ or $\left(V_{3}\right)^{\oplus 2} \oplus \mathbf{C}^{2}$. But then one of $H_{2}$ or $H_{3}$ acts trivially on $\operatorname{Spin}(6)$ and by $\left(V \oplus \mathbf{C}^{2}, \mathbf{C}^{4}\right)$ on $\operatorname{Sp}(4)$, contrary to what we arranged before the lemma.

It remains to consider the case where $H_{2} \times H_{3}$ acts on $\mathrm{Sp}(4)$ by $\left(V_{2} \oplus \mathbf{C}^{2},\left(V_{3}\right)^{\oplus 2}\right)$. In this case, $c_{2} V_{3}$ represents a generator of $H^{4}\left(S^{4}, \mathbf{Z}\right)$, while $c_{2} V_{2}$ is 2 times that generator. Going through the list of possible homomorphisms from $H_{2} \times H_{3}$ to $\operatorname{Spin}(6)$ again, we find that the only one whose Pontrjagin class in $H^{4}\left(S^{4}, \mathbf{Z}\right)$ is 2 times a generator, modulo 12 , is the one with complexification $\left(V_{3}\right)^{\oplus 2} \oplus \mathbf{C}^{2}$. But then $H_{2}$ acts trivially on $\operatorname{Spin}(6)$ and by $\left(V \oplus \mathbf{C}^{2}, \mathbf{C}^{4}\right)$ on $\operatorname{Sp}(4)$, contrary to what we arranged before the lemma.

q.e.d. 
We can now show that the case where $G_{1} / H_{1}$ is $\operatorname{Spin}(6) / \operatorname{Spin}(5)$ does not occur. In this case, we know that $H_{1}$ acts trivially on $\mathrm{Sp}(4)$ (which is isomorphic to Spin(5)), by Lemma 4.1. By Lemma 8.1, $H_{2} \times H_{3}$ does not act freely on $\mathrm{Sp}(4)$. On the other hand, the action of $H_{2} \times H_{3}$ on $\operatorname{Spin}(6)$ is given by one of the homomorphisms listed in the proof of Lemma 8.1. In all these cases, we check immediately that the maximal torus $\left(S^{1}\right)^{2}$ in $H_{2} \times H_{3}=\mathrm{SU}(2)^{2}$ has a fixed point in $\operatorname{Spin}(6) / \operatorname{Spin}(5)=S^{5}$. Therefore, there is a subgroup of $H=H_{1} \times H_{2} \times H_{3}$ which projects isomorphically to $\left(S^{1}\right)^{2}$ in $H_{2} \times H_{3}$ and which has a fixed point on $\operatorname{Spin}(6)$. Since $H$ acts freely on $G$, this subgroup acts freely on $\operatorname{Sp}(4)$. Since $H_{1}$ acts trivially on $\mathrm{Sp}(4)$, this means that the maximal torus $\left(S^{1}\right)^{2}$ in $H_{2} \times H_{3}$ acts freely on $\mathrm{Sp}(4)$. Since every element of $H_{2} \times H_{3}$ is conjugate to an element of the maximal torus, it follows that $H_{2} \times H_{3}$ acts freely on $\operatorname{Sp}(4)$, contradicting what we have shown. Thus the case where $G_{1} / H_{1}$ is $\operatorname{Spin}(6) / \operatorname{Spin}(5)$ does not occur.

It remains to consider the case where $G_{1} / H_{1}$ is $\mathrm{SU}(3) / \mathrm{SU}(2)=S^{5}$. That is, the 9 -manifold $N$ is a biquotient

$$
(\mathrm{SU}(3) \times \mathrm{Sp}(4)) / \mathrm{SU}(2)^{3},
$$

where we know that $H_{1}$ acts on $\mathrm{SU}(3)$ by the standard inclusion on one side, and we arranged earlier that neither $H_{2}$ nor $H_{3}$ acts trivially on one side of $\operatorname{Sp}(4)$ and by the standard inclusion on the other.

Suppose first that $H_{2}$ and $H_{3}$ act trivially on $G_{1}$. Then $H_{2} \times H_{3} \cong$ $\mathrm{SU}(2)^{2}$ must act freely on $\mathrm{Sp}(4)$. By Lemma 6.2 , up to switching $\mathrm{H}_{2}$ and $H_{3}$ and switching the two sides of $\mathrm{Sp}(4), H_{2} \times H_{3}$ acts on $\mathrm{Sp}(4)$ by the homomorphisms $\left(V_{2} \oplus \mathbf{C}^{2}, V_{3} \oplus V_{3}\right)$ or $\left(V_{2} \oplus V_{3}, \mathbf{C}^{4}\right)$. Therefore, one of the factors $\mathrm{H}_{2}$ or $\mathrm{H}_{3}$ acts on $\mathrm{Sp}(4)$ trivially on one side and by the standard inclusion on the other, as well as acting trivially on $\mathrm{SU}(3)$. This contradicts what we arranged earlier.

So one of $H_{2}$ or $H_{3}$ acts nontrivially on $G_{1}$. Switching $H_{2}$ and $H_{3}$ if necessary, we can assume that $H_{2}$ acts nontrivially on $G_{1}$. Since the centralizer of $H_{1}=\mathrm{SU}(2)$ in $G_{1}=\mathrm{SU}(3)$ is only finite by $S^{1}, H_{2}$ acts only on the other side of $G_{1}$ from $H_{1}$. It must act on $G_{1}=\mathrm{SU}(3)$ by the homomorphism $V_{2} \oplus \mathbf{C}$ or $S^{2} V_{2}$. Thus the centralizer of $H_{2}$ in $G_{1}$ is at most finite by $S^{1}$. Since the centralizer of $H_{1}$ on the other side of $G_{1}$ is finite by $S^{1}, H_{3}=\mathrm{SU}(2)$ must act trivially on $G_{1}$. By what we arranged earlier, it follows that $H_{3}$ does not act on $\mathrm{Sp}(4)$ trivially on one side and by the standard inclusion on the other.

Suppose first that $H_{2}$ acts on $\mathrm{SU}(3)$ by $V_{2} \oplus \mathbf{C}$. Then $H_{2}$ has a fixed point on $S^{5}$. More precisely, we see that the diagonal subgroup 
$\Delta_{12} \cong \mathrm{SU}(2)$ in $H_{1} \times H_{2}$ has a fixed point in $\mathrm{SU}(3)$. Since $H_{3}$ acts trivially on $G_{1}=\mathrm{SU}(3)$, it follows that $\Delta_{12} \times H_{3} \cong \mathrm{SU}(2)^{2}$ acts freely on $G_{2}=\mathrm{Sp}(4)$. By the classification of free actions of $\mathrm{SU}(2)^{2}$ on $\mathrm{Sp}(4)$ in Lemma 6.2, together with the fact that $H_{3}$ does not act trivially on one side of $\operatorname{Sp}(4)$ and by the standard inclusion on the other, $\Delta_{12} \times H_{3}$ must act on $\operatorname{Sp}(4)$ by the homomorphism $V_{12} \oplus \mathbf{C}^{2}$ on one side and $V_{3} \oplus V_{3}$ on the other. Here $V_{12}$ denotes the standard representation of $\Delta_{12}=\mathrm{SU}(2)$. By the Clebsch-Gordan formula, it follows that $H_{1} \times H_{2} \times H_{3}=\mathrm{SU}(2)^{3}$ acts on $\operatorname{Sp}(4)$ by $V_{1} \oplus \mathbf{C}^{2}$ or $V_{2} \oplus \mathbf{C}^{2}$ on one side and by $V_{3} \oplus V_{3}$ on the other. Switching $H_{1}$ and $H_{2}$ if necessary (which we can do, since they act the same way on $G_{1}=\mathrm{SU}(3)$ ), we can assume that $H_{1} \times H_{2} \times H_{3}=$ $\mathrm{SU}(2)^{3}$ acts on $\mathrm{Sp}(4)$ by $V_{2} \oplus \mathbf{C}^{2}$ on one side and by $V_{3}^{\oplus 2}$ on the other. Thus, $H_{1}$ acts trivially on $\mathrm{Sp}(4)$ and $H_{2} \times H_{3}$ acts freely on $\mathrm{Sp}(4)$, which contradicts Lemma 8.1.

Therefore $H_{2}=\mathrm{SU}(2)$ must act on $G_{1}=\mathrm{SU}(3)$ by the homomorphism $S^{2} V_{2}$. By Singhof's description of the tangent bundle of a biquotient $[25], w_{4} N$ is given by

$$
\begin{aligned}
w_{4} N & =w_{4} \mathrm{su}(3)+w_{4} \mathrm{sp}(4)-w_{4} \mathrm{su}(2)_{1}-w_{4} \operatorname{su}(2)_{2}-w_{4} \operatorname{su}(2)_{3} \\
& =w_{4} \mathrm{su}(3)+w_{4} \operatorname{sp}(4)
\end{aligned}
$$

using that $w_{4} \mathrm{su}(2)=0$ in $H^{4}\left(\mathrm{BSU}(2), \mathbf{F}_{2}\right)$. Furthermore, we know that $H_{2}$ acts on one side of $\mathrm{SU}(3)$ by $S^{2} V_{2}$, and no other factor of $H$ acts on that side of $\mathrm{SU}(3)$. Since $c_{2}\left(S^{2} V_{2}\right)=4 c_{2} V_{2}$, the generator $c_{2}$ of $H^{4}\left(\mathrm{BSU}(3), \mathbf{F}_{2}\right)$ pulls back to $4 c_{2} V_{2}=0$ in $H^{4}\left(N, \mathbf{F}_{2}\right)$. Therefore

$$
w_{4} N=w_{4} \operatorname{sp}(4) \text {. }
$$

We know that $H_{3}$ acts trivially on $\mathrm{SU}(3)$, so it must act freely on $\mathrm{Sp}(4)$. Furthermore, we know that it does not act trivially on one side of $\operatorname{Sp}(4)$ and by the standard inclusion $V_{3} \oplus \mathbf{C}^{2}$ on the other. By Lemma 6.2, $H_{3}$ must act on at least one side of $\operatorname{Sp}(4)$ by the homomorphism $V_{3}^{\oplus 2}$ or $S^{3} V_{3}$. These two homomorphisms have centralizers in $\operatorname{Sp}(4)$ which are finite by $S^{1}$ or finite, so no other simple factor of $H$ acts on the same side of $\operatorname{Sp}(4)$. Since $c_{2}\left(V_{3}^{\oplus 2}\right)=2 c_{2} V_{3}$ and $c_{2}\left(S^{3} V_{3}\right)=10 c_{2} V_{3}$, the generator $c_{2}$ of $H^{4}\left(\mathrm{BSp}(4), \mathbf{F}_{2}\right)$ pulls back to $2 c_{2} V_{3}$ or $10 c_{2} V_{3}$ in $H^{4}\left(N, \mathbf{F}_{2}\right)$, thus to zero. Therefore

$$
w_{4} N=0,
$$

contradicting what we know about $N$. This completes the proof that $\mathbf{C P}^{4} \# \mathbf{H P}^{2}$ is not homotopy equivalent to a biquotient. Theorem 2.1 is proved.

q.e.d. 


\section{References}

[1] J.F. Adams \& M. Atiyah, K-theory and the Hopf invariant, Quart. J. Math. 17 (1966) 31-38, MR 33 \#6618, Zbl 0136.43903.

[2] R. Aguilar, Symplectic reduction and the complex homogeneous Monge-Ampère equation, Ann. Global Anal. Geom. 19 (2001) 327-353, MR 2002g:53144, Zbl 1001.53058.

[3] M. Berger, Les variétés riemanniennes homogènes normales simplement connexes à courbure strictement positive, Ann. Scuola Norm. Sup. Pisa 15 (1961) 179-246, MR 24 \#A2919, Zbl 0101.14201.

[4] A. Borel, Le plan projectif des octaves et les sphères comme espaces homogènes, C.R. Acad. Sci. 230 (1950) 1378-1380, MR 11,640c, Zbl 0041.52203.

[5] N. Bourbaki, Groupes et algèbres de Lie, Chapitres 4, 5 et 6, Masson, 1981, MR 39 \#1590, Zbl 0186.33001.

[6] J. Cheeger, Some examples of manifolds of nonnegative curvature, J. Differential Geom. 8 (1973) 623-628, MR 49 \#6085, Zbl 0281.53040.

[7] A. Dold, Erzeugende der Thomschen Algebra R, Math. Zeit. 65 (1956) 25-35, MR 18,60c, Zbl 0071.17601.

[8] E. Dynkin, Semisimple subalgebras of semisimple Lie algebras, Mat. Sbornik 30 (1952) 349-462, MR 13,904c; Amer. Math. Soc. Translations (2) 6 (1957) 111244, Zbl 0077.03404.

[9] J. Eschenburg, New examples of manifolds with strictly positive curvature, Invent. Math. 66 (1982) 469-480, MR 83i:53061, Zbl 0484.53031.

[10] J. Eschenburg, Freie isometrische Aktionen auf kompakten Lie-Gruppen mit positiv gekrümmten Orbiträumen, Schriften der Math. Universität Münster 32, 1984, MR 86a:53045, Zbl 0551.53024.

[11] J. Eschenburg, Cohomology of biquotients, Manu. Math. 75 (1992) 151-166, MR 93e:57070, Zbl 0769.53029.

[12] Y. Félix, S. Halperin \& J.-C. Thomas, Elliptic spaces II, Ens. Math. 39 (1993) 25-32, MR 94f:55008, Zbl 0786.55006.

[13] Y. Félix, S. Halperin \& J.-C. Thomas, Rational homotopy theory, Springer, 2001, MR 2002d:55014, Zbl 0961.55002.

[14] V. Gorbatsevich \& A. Onishchik, Lie transformation groups, 95-229, in 'Lie groups and Lie algebras I', ed. A. Onishchik, Encyclopaedia of Math. Sciences, 20, Springer, 1993, MR 1306 739, Zbl 0781.22004.

[15] D. Gromoll \& W. Meyer, An exotic sphere with nonnegative sectional curvature, Ann. Math. 100 (1974), 401-406, MR 51 \#11347, Zbl 0293.53015. 
[16] K. Grove \& W. Ziller, Curvature and symmetry of Milnor spheres, Ann. Math. 152 (2000), 331-367, MR 2001i:53047, Zbl 0991.53016.

[17] V. Kapovitch \& W. Ziller, Biquotients with singly generated rational cohomology, to appear in Geometriae Dedicata, math.DG/0210231.

[18] P. Lambrechts, The Betti numbers of the free loop space of a connected sum, J. LMS 64 (2001) 205-228, MR 2002f:55022.

[19] J. Milnor \& J. Stasheff, Characteristic classes, Princeton, 1974, MR 55 \#13428, Zbl 0298.57008.

[20] A. Onishchik, Inclusion relations among transitive compact transformation groups, Trudy Moskov. Mat. Obshch. 11 (1962) 199-242; Amer. Math. Soc. Translations (2) $\mathbf{5 0}$ (1966) 5-58, Zbl 0207.33604.

[21] A. Onishchik, Remark on invariants of groups generated by reflections, Selecta Math. Sov. 3 (1983/84), 239-241, MR 87h:20075, Zbl 0541.20028.

[22] A. Onishchik, Topology of transitive transformation groups, Johann Ambrosius Barth, Leipzig, 1994, MR 95e:57058, Zbl 0796.57001.

[23] G. Paternain, Differentiable structures with zero entropy on simply connected 4manifolds, Bol. Soc. Brasil. Mat. 31 (2000) 1-8, MR 2001c:53042.

[24] A. Petrunin \& W. Tuschmann, Diffeomorphism finiteness, positive pinching, and second homotopy, Geom. Funct. Anal. 9 (1999) 736-774, MR 2000k:53031, Zbl 0941.53026.

[25] W. Singhof, On the topology of double coset manifolds, Math. Ann. 297 (1993) 133-146, MR 94k:57054, Zbl 0793.57019.

[26] B. Totaro, Complexifications of nonnegatively curved manifolds, J. Eur. Math. Soc. 5 (2003), 69-94.

[27] B. Totaro, Curvature, diameter, and quotient manifolds, Math. Res. Lett., to appear.

[28] W.-T. Wu, On Pontrjagin classes III, Amer. Math. Soc. Translations (2) 11 (1959) 155-172, MR 22 \#5981, Zbl 0084.19004.

DPMMS, WILBERForCE RoAD Cambridge CB3 0WB, England 